\title{
The pre-inflationary dynamics of loop quantum cosmology: Confronting quantum gravity with observations
}

\author{
Ivan Agullo ${ }^{1,2},{ }^{*}$ Abhay Ashtekar ${ }^{1}{ }^{\dagger}$ and William Nelson ${ }^{1,3 \ddagger}$ \\ 1 Institute for Gravitation and the Cosmos 8 Physics Department, \\ Penn State, University Park, PA 16802, U.S.A. \\ 2 Center for Theoretical Cosmology, DAMTP, Wilberforce Road, \\ University of Cambridge, Cambridge CB3 OWA, U.K. and \\ 3 Radboud University Nijmegen, Institute for Mathematics, \\ Astrophysics and Particle Physics, Heyendaalsweg 135,6525-AJ Nijmegen, Netherlands \\ Using techniques from loop quantum gravity, the standard theory of cosmological \\ perturbations was recently generalized to encompass the Planck era. We now apply \\ this framework to explore pre-inflationary dynamics. The framework enables us to \\ isolate and resolve the true trans-Planckian difficulties, with interesting lessons both \\ for theory and observations. Specifically, for a large class of initial conditions at the \\ bounce, we are led to a self consistent extension of the inflationary paradigm over \\ the 11 orders of magnitude in density and curvature, from the big bounce to the \\ onset of slow roll. In addition, for a narrow window of initial conditions, there are \\ departures from the standard paradigm, with novel effects - such as a modification \\ of the consistency relation between the ratio of the tensor to scalar power spectrum \\ and the tensor spectral index, as well as a new source for non-Gaussianities - which \\ could extend the reach of cosmological observations to the deep Planck regime of the \\ early universe.
}

\section{INTRODUCTION}

Thanks to a powerful interplay between theory and observations, our understanding of the early universe has advanced very significantly in recent years. Consequently, cosmology offers a natural arena to develop and test approaches to quantum gravity.

In this paper we will focus on inflation, the leading scenario to successfully account for the tiny inhomogeneities observed in the cosmic microwave background (CMB) (see, e.g., [1-5]). An impressive feature of this scenario is that it involves a rather small set of assumptions. As noted in $[6,7]$, these can be summarized as follows:

- Sometime in its early history, the universe underwent a phase of rapid expansion during which the Hubble parameter was nearly constant;

- During this phase, the universe was well described by a Friedman, Lemaître, Robertson

\footnotetext{
*Electronic address: I.AgulloRodenas@damtp.cam.ac.uk

${ }^{\dagger}$ Electronic address: ashtekar@gravity.psu.edu

${ }_{\ddagger}^{\ddagger}$ Electronic address: nelson@gravity.psu.edu
} 
Walker (FLRW) solution to Einstein's equations with a scalar field as matter source, together with small inhomogeneities which are well approximated by first order perturbations;

- Fourier modes of the quantum fields representing perturbations were initially in the Bunch-Davies (BD) vacuum at least for a certain set of wave numbers ${ }^{1}$; and,

- Soon after any mode exits the Hubble radius, its quantum fluctuation can be regarded as a classical perturbation and evolved via linearized Einstein's equations.

Analysis of these inflationary perturbations implies that there must be tiny inhomogeneities at the surface of last scattering whose detailed features have been observed in the CMB. Furthermore, these tiny inhomogeneities serve as seeds that grow into a large scale structure which is in excellent qualitative agreement with observations. Therefore, even though the underlying assumptions are by no means 'obvious' or 'compelling', the success of the subsequent predictions is impressive.

Yet, as is well known (see e.g. [8]), the scenario is conceptually incomplete in several respects. In particular, as Borde, Guth and Vilenkin [9] showed, inflationary space-times inherit the big-bang singularity. Physically, this occurs because one continues to use general relativity even in the Planck regime in which it is simply not applicable. It is widely expected that new physics in this regime will resolve the singularity, significantly changing the very early history of the universe. Will inflation arise naturally in the resulting deeper theory? Or, more modestly, can one at least obtain a consistent quantum gravity extension of this scenario?

To fully address this question one has to face two distinct sets of issues. The first set has its origin in particle physics. What is the physical origin of the scalar field that plays the role of the inflaton? It should arise naturally in the fundamental unified theory. Can we derive the inflationary potential from some first principles? Is there a single inflaton, as in the simplest models that have been successful so far, or many? How do they interact with one another? Even more importantly, what are the interactions that produce the elementary constituents of the standard model of particle physics during reheating? These issues remain open, but fall in the realm of theories aimed at unification. In this and the companion papers, we do not address them.

Rather, we will continue to use the simplest, single inflaton model and focus on the second set of issues related to gravity. In the systematic evolution starting from the Planck regime, does a slow roll phase compatible with the WMAP data arise generically in the background geometry, or, is an enormous fine tuning needed? In classical general relativity, if we evolve the modes of observational interest back in time, they become trans-Planckian at some stage. Is there a quantum field theory ( $\mathrm{QFT}$ ) on quantum space-times that is needed to adequately handle physics at that stage? Can one arrive at the $\mathrm{BD}$ vacuum at the onset of the slow roll from more fundamental considerations? Or, is an even more elaborate fine tuning of the quantum state of perturbations necessary in the Planck regime? The natural state of quantum perturbations at the onset of inflation may be indistinguishable from the $\mathrm{BD}$ vacuum for currently available data but, because of the pre-inflationary dynamics especially

\footnotetext{
${ }^{1}$ More precisely, if $k_{o}$ denotes the co-moving wave number of the mode which has just re-entered the Hubble radius now then, at the time $t\left(k_{o}\right)$ at which this mode exited the Hubble radius during inflation, Fourier modes with co-moving wave numbers in the range $\sim\left(k_{o}, 2000 k_{o}\right)$ were in the Bunch-Davies vacuum.
} 
in the Planck regime, it could well carry certain excitations over the $\mathrm{BD}$ vacuum. These can then source non-Gaussianities for the subsequent evolution during inflation [10-14] which, in turn, could give rise to novel effects in the CMB and galaxy distribution [15-17] which could be observed in future missions. Can the state at the onset of the slow roll be close enough to the $\mathrm{BD}$ vacuum to agree with current observations and yet be sufficiently different to give rise to such effects?

This is the third in a series of papers whose goal is to address these questions that originate in quantum gravity. The first paper [18] presented a succinct summary of underlying framework and the principal observational consequences for a broad audience. The second [19] was primarily addressed to the quantum gravity community and focused on conceptual and mathematical aspects of the framework. In this paper we use that framework to analyze phenomenological issues of interest to cosmologists within the standard inflationary scenario.

The main ideas can be summarized as follows.

By now there is a large body of results in loop quantum cosmology (LQC) that provides a detailed description of the Planck scale physics in a number of cosmological models. These include the $\mathrm{k}=0$ and $\mathrm{k}=1$ (FLRW) models [20-28], possibly with a non-zero cosmological constant [29-31], the anisotropic Bianchi I, II and IX models [32-35] and the simplest of the inhomogeneous models - the Gowdy space-times - widely studied in exact general relativity [36-40]. In all cases, the big bang singularity is resolved and replaced by quantum bounces (for a review, see, e.g., $[7,41]$ ). Thus, the first conceptual limitation of the standard general relativistic models is overcome. It is therefore natural to use LQC as the point of departure for extending the cosmological perturbation theory. How do new effects in the Planck domain - such as the robust superinflation phase that follows the density bounce - alter the standard inflationary scenario? Do generic initial conditions at the bounce lead to the desired slow roll inflation later, or, is a fine tuning necessary?

To incorporate cosmological perturbations, however, one cannot just mimic the standard procedure used in general relativity: Since loop quantum gravity (LQG) is still incomplete, we do not yet have the analog of full Einstein's equations to perturb. A number of LQC inspired strategies have been devised to overcome this obstacle. ${ }^{2}$ In this series of papers we follow a mainstream strategy in LQG: First truncate the classical theory in a manner appropriate to the physical problem under consideration, then carry out quantization paying due attention to the underlying quantum geometry of $L Q G$, and finally work out the consequences of the resulting framework. This procedure has been successful not only in the cosmological models referred to above but also in the investigation of quantum black holes in LQG [42-44] and derivation of the graviton propagator using spin foams [45, 46].

For the present application, we will truncate the full phase space, keeping only the FLRW backgrounds with first order inhomogeneous perturbations. To facilitate easy comparison with the standard quantum theory of cosmological perturbations, we will employ a hybrid scheme used in [36-40, 47], in which one uses the standard LQC for homogeneous modes and 'Fock-like' states for the first order perturbations. There is however a key difference from the standard cosmological perturbation theory: Because the background space-time now has quantum geometry, we are led to use QFT on quantum cosmological space-times. This theory was introduced in [48] emphasizing conceptual issues and developed in [19] to incorporate the infinitely many modes of perturbations and the associated regularization

\footnotetext{
${ }^{2}$ For a short summary of the underlying ideas, merits and limitations, see section II.A in [19].
} 
and renormalization techniques which, as discussed below, are key to checking the selfconsistency of our initial truncation. Since we only have a probability amplitude for various FLRW geometries - rather than a sharply defined classical FLRW metric - at a fundamental level evolution has to be described using a relational time variable rather than cosmic or conformal time. Thus, quantum gravity introduces genuinely new conceptual elements. But one can systematically 'descend' to a description in terms of cosmic and conformal time of an effective metric that incorporates the quantum gravity corrections.

Since quantum perturbations now propagate on quantum geometries which are all regular, free of singularities, the framework automatically encompasses the Planck regime. What is the status of the 'trans-Planckian issue' which, in heuristic discussions, is often associated with modes of trans-Planckian frequencies? Recall, first, that the quantum Riemannian geometry underlying LQG is subtle $[49,50]$. For example, while there is a minimum nonzero eigenvalue of the area operators, there is no such minimum for the volume operators, even though their eigenvalues are also discrete $[51,52]^{3}$ As a consequence, there is no fundamental obstacle preventing trans-Planckian modes of perturbations in our truncated theory. Indeed, in the homogeneous LQC models that have been analyzed in detail, the momentum $p_{(\phi)}$ of the scalar field $\phi$ is generally huge in Planck units. This poses no problem and, in particular, on the physical Hilbert space the total energy density is still guaranteed to be bounded by $0.41 \rho_{\mathrm{Pl}}$ where $\rho_{\mathrm{Pl}}$ is the Planck density. Similarly, for perturbations, there is no a priori difficulty with trans-Planckian momenta (or frequencies) in the truncated theory considered here. The real danger is rather that, in presence of such modes, the energy density in perturbations may fail to be negligible compared to that in the quantum background geometry. This is a very non-trivial issue especially in the Planck regime following the bounce and requires a careful treatment of regularization and renormalization of the stress energy tensor of quantum perturbations. If the energy density does become comparable to that in the background, then we would not be able to neglect the back-reaction and our truncation would fail to be self-consistent. This is the trans-Planckian problem we face in our theory of quantum perturbations on inflationary quantum geometries.

The paper is organized as follows. Section II summarizes the underlying conceptual and mathematical framework (constructed in [19] using prior results from [6, 22-25, 56, 57]) for the cosmology community. This discussion encompasses the scalar as well as the tensor modes. Section III discusses the initial conditions for the wave function $\Psi_{o}$ representing the background quantum geometry, and for the wave function $\psi$ representing the perturbations propagating on the quantum geometry $\Psi_{o}$. The choices can be motivated by general symmetry principles and physical considerations. Sections IV and V present results of numerical simulations of the pre-inflationary dynamics for the quantum corrected background and perturbations. The free parameter that dictates the evolution of the background is the value $\phi_{\mathrm{B}}$ of the inflaton at the bounce. In section $\mathrm{V}$ we vary this parameter (within a range that is numerically feasible) and explore consequences on the observed power spectrum at the end of inflation. We find an unforeseen and interesting structure. The LQC power spectrum is essentially independent of the value of $\phi_{\mathrm{B}}$. But recall that there is only a finite interval in the space of co-moving wave numbers $k$ that is relevant for the CMB observations. This interval is completely determined by the value of $\phi_{\mathrm{B}}$, moving steadily to the right along the $k$-axis as $\phi_{\mathrm{B}}$ increases. If $\phi_{\mathrm{B}}$ is sufficiently low, the quantum state at the onset of the

\footnotetext{
${ }^{3}$ Properties of the eigenvalues of length operators [53-55] have not been analyzed in comparable detail. But since their definitions involve volume operators, it is expected that there would be no 'length gap'.
} 
slow roll is sufficiently different from the BD vacuum to lead to observable effects for future measurements along the lines of [15-17].

In section VI we analyze the issue of self consistency of the truncation strategy: Does the stress energy in the perturbations remain small compared to that in the background throughout the evolution from the big bounce to the onset of the slow roll? The main result is that the self-consistency criterion is met for a large class of initial conditions. In these cases, our main approximation - the initial truncation - is viable. Had it failed, it would have been inconsistent to keep only the first order perturbations. Then one would have had to wait for significant advances in full LQG to extend the inflationary paradigm to the quantum gravity regime. Thus, technically as well as conceptually it is quite non-trivial that this does not happen and one obtains a self consistent extension of the standard inflationary paradigm all the way to the deep Planck regime of the big bounce for a wide range of initial conditions.

Of course, self-consistency by itself does not imply that our truncated solution is necessarily close to an exact one (because the sum of all higher order terms could be large). But this limitation is common to all perturbation theories, classical or quantum. In particular, in classical cosmology the total back reaction is routinely neglected if stress-energy in the first order perturbations is small compared to that in the background, even though strictly this does not imply that there is an exact solution close to the linearized one. If the first order perturbations do not grow so much in time as to become comparable to the background, the test field approximation is self consistent and is regarded as trustworthy. We adopt the same viewpoint here.

In section VII we summarize the main results and discuss open issues. The Appendix summarizes the technical differences between Ref. [19] and the underlying framework presented in section II which is tailored to the inflation.

We will use the following conventions. The signature of the space-time metric will be ,,,-+++ . We set $c=1$ but keep $G$ and $\hbar$ explicitly in various equations to facilitate the distinction between classical and quantum effects. As is usual, we will set $\kappa=8 \pi G$ and, for the Barbero Immirzi parameter $\gamma$ of LQG, use the value $\gamma \approx 0.24$ that comes from the black hole entropy calculations. Finally, we will use Planck units used in the quantum gravity literature rather than the reduced Planck units often used in cosmology. (Thus, our Planck mass $m_{\mathrm{Pl}}=\sqrt{\hbar / G}$ is related to the reduced Planck mass $M_{\mathrm{Pl}}$ via $m_{\mathrm{Pl}}=\sqrt{8 \pi} M_{\mathrm{Pl}}$.) Numerical values of all quantities are given in dimensionless Planck units $\ell_{\mathrm{Pl}}=m_{\mathrm{Pl}}=t_{\mathrm{Pl}}=$ 1.

\section{THE UNDERLYING FRAMEWORK}

In discussions of the early universe, one generally begins with a FLRW solution to Einstein's equation with suitable matter fields as sources, then considers the space of solutions to the first order perturbation equations on this background space-time, and finally quantizes them using standard techniques of QFT in curved space-times. To incorporate the Planck regime, the FLRW background must also be treated quantum mechanically. Therefore, we cannot begin with a solution to Einstein's equations. Instead, we will first introduce a Hamiltonian framework that encompasses both the FLRW backgrounds and first order perturbations thereon and then pass to the quantum theory of the combined system as a whole. As explained in section I, this will lead us to a quantum theory of fields representing linearized perturbations, propagating on a quantum FLRW geometry. 
We will assume that the spatial topology is $\mathbb{R}^{3}$ and focus on the $k=0$ FLRW case. As in the standard inflationary models, the matter field will be taken to be a scalar field and for detailed calculations we will use the simplest potential $V(\phi)=(1 / 2) m^{2} \phi^{2}$. Most of the results discussed in this section are generalizations of those discussed in detail in [19], now allowing for a inflaton potential $V(\phi)$ and $\mathbb{R}^{3}$ spatial topology (in place of a 3 -torus).

\section{A. The truncated phase space}

Let us first truncate the full phase space of general relativity to the physical problem of interest. This truncated phase space $\Gamma_{\text {Trun }}$ is of the form $\Gamma_{\text {Trun }}=\Gamma_{o} \times \tilde{\Gamma}_{1}$ where $\Gamma_{o}$ is the 4-dimensional phase space for the FLRW backgrounds and $\tilde{\Gamma}_{1}$ of gauge invariant perturbations.

Because the background fields are homogeneous and the spatial topology is $\mathbb{R}^{3}$, the spatial integrals that appear in the expressions of the symplectic structure and Hamiltonians on $\Gamma_{o}$ diverge. Therefore, in the construction of the Hamiltonian framework of the homogeneous sector, one first fixes co-moving Cartesian coordinates $x^{a}$, introduces an elementary cell $\mathcal{C}$ whose edges are aligned along these coordinates and have equal coordinate lengths $\ell$, and restricts all integrations to $\mathcal{C}$. This is an infrared cut-off for the homogenous sector which is removed at the end by letting $\ell$ tend to infinity [7].

In LQC, in place of the scale factor $a$ and its conjugate momentum $\pi_{(a)}$, it is customary to use the following pair:

$$
\nu=\frac{4 a^{3} \ell^{3}}{\kappa \gamma \hbar} ; \quad \text { and } \quad \mathrm{b}=-\frac{\gamma \kappa}{6 a^{2} \ell^{3}} \pi_{(a)}
$$

so that the fundamental Poisson bracket is given by $\{\mathrm{b}, \nu\}=\frac{2}{\hbar}$. Thus, apart from constants, $\nu$ gives the physical volume of the fiducial cell $\mathcal{C}$ and, on solutions to Einstein's equations, $\mathrm{b}$ is related to the Hubble parameter $H=\dot{a} / a$ via $\mathrm{b}=\gamma H$. The homogeneous scalar field and its momentum will be denoted by $\phi$ and $p_{(\phi)}$ respectively. Thus the background FLRW phase space $\Gamma_{o}$ is coordinatized by $\left(\nu, \mathrm{b} ; \phi, p_{(\phi)}\right)$ and carries a single scalar (or Hamiltonian) constraint:

$$
\mathbb{S}_{o}\left[N_{\text {hom }}\right]=N_{\text {hom }}\left[-\frac{3 \hbar \mathrm{b}^{2} \nu}{4 \gamma}+\frac{2 p_{(\phi)}^{2}}{\kappa \gamma \hbar \nu}+\frac{\kappa \gamma \hbar \nu}{4} V(\phi)\right]=0
$$

where $N_{\text {hom }}$ represents a homogeneous lapse. Einstein dynamics is generated by this scalar constraint. The choice $N_{\text {hom }}=1$ yields evolution in cosmic time; $N_{\text {hom }}=a$ in conformal time; and $N_{\text {hom }}=a^{3}$ in harmonic time. ( $N_{\text {hom }}=a^{3} \ell^{3} / p_{(\phi)}$ corresponds to using the inflaton $\phi$ as time in 'patches' of dynamical trajectories along which it is monotonic.)

The phase space $\tilde{\Gamma}_{1}$ of gauge invariant perturbations is spanned by 3 canonically conjugate pairs, one representing the Mukhanov-Sasaki scalar mode and two representing the tensor modes. It is simplest to work in the (co-moving) momentum space and represent them by

$$
\left(\mathcal{Q}_{\vec{k}}, \mathcal{T}_{\vec{k}}^{(1)}, \mathcal{T}_{\vec{k}}^{(2)} ; \mathfrak{p}_{\vec{k}}^{\mathcal{Q}}, \mathfrak{p}_{\vec{k}}^{\mathcal{T}_{1}}, \mathfrak{p}_{\vec{k}}^{\mathcal{T}_{2}}\right)
$$

Since the perturbations are not homogeneous, in the discussion of $\tilde{\Gamma}_{1}$ one can work directly with the entire $\mathbb{R}^{3}$; restriction to the cell $\mathcal{C}$ is not necessary. We choose this route to avoid an artificial quantization of the momenta $\vec{k}$ that the restriction to the cell would have 
introduced. Technically, we assume that the perturbations are square-integrable so that one can freely pass between $\vec{x}$ and $\vec{k}$ spaces. Then the Poisson bracket relations are given by $\left\{\mathcal{Q}_{\vec{k}}, \mathfrak{p}_{\vec{k}^{\prime}}^{(\mathcal{Q})}\right\}=(2 \pi)^{3} \delta\left(\vec{k}+\vec{k}^{\prime}\right)$, and similarly for tensor modes. For simplicity of notation, we will often use collective labels $\mathcal{T}_{\vec{k}}, \mathfrak{p}_{\vec{k}}^{(\mathcal{T})}$ for the two tensor modes.

On the truncated phase space $\Gamma_{\text {Trun }}$, the dynamical trajectories follow integral curves of the 'evolution vector field' $X_{\text {Dyn }}^{\alpha}=\Omega_{o}^{\alpha \beta} \partial_{\beta} \mathbb{S}_{o}+\Omega^{\alpha \beta} \partial_{\beta} \mathbb{S}_{2}^{\prime}$ where $\Omega_{o}$ is the symplectic structure on $\Gamma_{o}, \Omega_{1}$ on $\tilde{\Gamma}_{1}$ and $\mathbb{S}_{o}$ is defined in Eq (2.2). The function $\mathbb{S}_{2}^{\prime}$ governing the time evolution of perturbations is obtained from the second order truncation $\mathbb{S}_{2}$ of the full scalar constraint of general relativity by keeping only the terms which are quadratic in first order perturbations and deleting the term that is linear in the second order perturbations. ${ }^{4}$ It is given by $\mathbb{S}_{2}^{\prime}=\mathbb{S}_{2}^{\prime(\mathcal{Q})}+\mathbb{S}_{2}^{\prime \mathcal{T}_{1}}+\mathbb{S}_{2}^{\prime \mathcal{T}_{2}}$, with

$$
\mathbb{S}_{2}^{\prime(\mathcal{T})}\left[N_{\text {hom }}\right]=\frac{N_{\text {hom }}}{2} \int \frac{d^{3} k}{(2 \pi)^{3}}\left(\frac{4 \kappa}{a^{3}}\left|\mathfrak{p}_{\vec{k}}^{(\mathcal{T})}\right|^{2}+\frac{a k^{2}}{4 \kappa}\left|\mathcal{T}_{\vec{k}}\right|^{2}\right)
$$

and

$$
\mathbb{S}_{2}^{\prime(\mathcal{Q})}\left[N_{\mathrm{hom}}\right]=\frac{N_{\mathrm{hom}}}{2} \int \frac{d^{3} k}{(2 \pi)^{3}}\left(\frac{1}{a^{3}}\left|\mathfrak{p}_{\vec{k}}^{(\mathcal{Q})}\right|^{2}+a\left(\mathfrak{A}+k^{2}\right)\left|\mathcal{Q}_{\vec{k}}\right|^{2}\right) .
$$

In (2.5), $\mathfrak{A}$ is a function only of the background variables (and therefore has no $\vec{k}$ dependence):

$$
\mathfrak{A}=\left(\mathfrak{f} V(\phi)-2 \sqrt{\mathfrak{f}} V_{, \phi}+V_{, \phi \phi}\right) a^{2}
$$

with $\mathfrak{f}=\left(3 \kappa p_{(\phi)}^{2}\right) /\left((1 / 2) p_{(\phi)}^{2}+\ell^{6} a^{6} V(\phi)\right)$ (see Appendix (A). Since $p_{(\phi)}=a^{3} \ell^{3} \dot{\phi}$, the quantity $\mathfrak{f}$ is essentially the kinetic fraction of the total energy density.)

The resulting time evolution of tensor modes is the same as that of a massless scalar field on a given FLRW background. By contrast, the scalar mode experiences an external, time dependent potential $\mathfrak{A}$ in addition to the FLRW geometry. If $V(\phi)$ were to vanish, we would have $\mathfrak{A}=0$ and then the dynamics of the (trivially rescaled) scalar mode $\mathcal{Q}_{\vec{k}} / 2 \sqrt{\kappa}$ would be the same as that of the tensor mode $\mathcal{T}_{\vec{k}}$ (as in [19]).

Recall first that the time evolution in the FLRW sector is generated by the restriction $\mathbb{S}_{o}$ to the FLRW subspace $\Gamma_{o}$ of the Hamiltonian constraint on the full phase space $\Gamma$ of general relativity. This evolution is reproduced by the first term $\Omega_{o}^{\alpha \beta} \partial_{\beta} \mathbb{S}_{o}$ of the dynamical vector field $X_{\text {Dyn }}^{\alpha}$. Consequently these dynamical trajectories are solutions to the full Einstein equations. However in the second part of the dynamical vector field, $\mathbb{S}_{2}^{\prime}$ is not constrained to vanish. Indeed, even in the second order truncation of the full constraint on $\Gamma$, it is $\mathbb{S}_{2}$ that vanishes, not $\mathbb{S}_{2}^{\prime}$. The fact that dynamics is governed by a constraint only on $\Gamma_{o}$ and not on the full $\Gamma_{\text {Trun }}$ has an important consequence in the quantum theory.

\footnotetext{
${ }^{4}$ Here the Greek indices refer to the (infinite dimensional) tangent space to $\Gamma_{\text {Trun }}$. In terms of Poisson brackets, the evolution of functions $F$ of background quantities $\nu, \mathrm{b}, \phi, p_{(\phi)}$ is given by $\dot{F}=\left\{F, \mathbb{S}_{o}\right\}_{o}$, where the 'dot' denotes derivative with respect to the time variable determined by the lapse and the Poisson bracket is taken only with respect to the background variables. The evolution of functions $f$ of gauge invariant perturbations $\left(\mathcal{Q}_{\vec{k}}, \mathcal{T}_{\vec{k}} ; \mathfrak{p}_{\vec{k}}^{(\mathcal{Q})}, p_{\vec{k}}^{(\mathcal{T})}\right)$ is given by $\dot{f}=\left\{f, \mathbb{S}_{2}^{\prime}\right\}_{1}$ where the Poisson bracket is taken only with respect to the perturbations, treating the background quantities in the expression of $\mathbb{S}_{2}^{\prime}$ (and possibly $f$ ) as given time-dependent variables determined by first solving the evolution equation for functions $F$.
} 
Finally, because $\mathbb{S}_{2}^{\prime}$ depends not only on perturbations but also on the background variable $\nu$ (i.e. the scale factor), the dynamical vector field is not of the form $\left(\Omega_{o}^{\alpha \beta}+\Omega_{1}^{\alpha \beta}\right) \partial_{\beta} H$ for any function $H$ on $\Gamma_{\text {Trun }}$ : In striking contrast to full general relativity, or its FLRW sector, dynamics is not generated by any Hamiltonian on $\Gamma_{\text {Trun }}$. In the phase space language, we have to first obtain the dynamical trajectory on $\Gamma_{o}$ and then lift it to the truncated phase space $\Gamma_{\text {Trun }}$ using integral curves of $X_{\text {Dyn }}^{\alpha}$. This procedure just reflects the steps normally followed using space of solutions: one first solves for the background scale factor $a(\eta)$ and the scalar field $\phi(\eta)$ using FLRW equations, fixes a specific solution as the background space-time and then solves for perturbation equations on this background. Therefore, in the quantum theory we are led to first solve for the background wave function $\Psi_{o}(\nu, \phi)$ and then 'lift this quantum trajectory' to a wave function $\Psi_{o} \otimes \psi\left(\mathcal{Q}_{\vec{k}}, \mathcal{T}_{\vec{k}}, \phi\right)$ describing the evolution of the quantum wave function $\psi$ on the quantum background geometry $\Psi_{o}$.

\section{B. Quantum FLRW geometry in LQC}

Since the phase space of the truncated system is a product, $\Gamma_{\text {Trun }}=\Gamma_{o} \times \tilde{\Gamma}_{1}$, the Hilbert space of quantum states has the form $\mathcal{H}=\mathcal{H}_{o} \otimes \mathcal{H}_{1}$. In this sub-section we will focus on the space $\mathcal{H}_{o}$ of quantum states of the background geometry and the metric operator thereon. The sector $\mathcal{H}_{1}$ of perturbations will be discussed in the next sub-section.

\section{Quantum dynamics of the FLRW background}

Although we have used background variables tailored to LQC, the classical Hamiltonian theory is the same as in general relativity. It is in the passage to the quantum theory that we use the LQG techniques. For simplicity, we will first summarize the basic ideas and return to two important technical points at the end.

Recall that $\Gamma_{o}$ is spanned by two canonically conjugate pairs $\left(\nu, \phi ; \mathrm{b}, p_{(\phi)}\right)$, and carries a scalar constraint $\mathbb{S}_{o}(\nu, \mathrm{b})=0$ (see $(2.2)$ ). Therefore quantum states are represented by wave functions $\Psi_{o}(\nu, \phi)$ and the Dirac quantization procedure would lead us to impose $\hat{\mathbb{S}}_{o} \Psi_{o}=0$, which takes the form $\left(\hbar^{2} \partial_{\phi}^{2}+\Theta\right) \Psi_{o}=0$ for a specific operator $\Theta$. A careful analysis [58-60] of constrained systems implies that the physical Hilbert space $\mathcal{H}_{o}$ is spanned by states that satisfy a mathematically 'sharper' Hamiltonian constraint, which can be intuitively thought of as the 'positive frequency square-root' of $\hat{\mathbb{S}}_{o} \Psi_{o}=0[23]$ :

$$
-i \hbar \partial_{\phi} \Psi_{o}(\nu, \phi)=\hat{H}_{o} \Psi_{o}(\nu, \phi)
$$

where $\hat{H}_{o}$ is a self-adjoint operator whose explicit form will not be needed in this summary. Thus, the constraint is 'de-parameterized': its form suggests that we can interpret the scalar field $\phi$ as an 'internal' or a 'relational' time variable (a la Leibniz) with respect to which the 'true' dynamical variable $\hat{\nu}$ (and also the perturbations $\hat{\mathcal{Q}}_{\vec{k}}, \hat{\mathcal{T}}_{\vec{k}}$ ) evolve. Thus, in the physical sector of the theory, $\phi$ is just a parameter; there is no longer an operator associated with it. Now, given $\Psi_{o}\left(\nu, \phi_{\mathrm{B}}\right)$ at the bounce time $\phi=\phi_{\mathrm{B}}$, Eq (2.7) enables one to 'evolve' this initial state to obtain the quantum state $\Psi_{o}(\nu, \phi)$ at all times $\phi$. This quantum evolution is nonsingular; the wave function undergoes a bounce when the density reaches a maximum value $\rho_{\max } \approx 0.41 \rho_{\mathrm{Pl}}$. How does LQC evade the standard singularity theorems? The singularity theorem due to Borde, Guth and Vilenkin [9], tailored to inflation, is evaded because the 
LQC universe has a contacting phase in the past, violating their assumption of eternal expansion. And LQC bypasses the original singularity theorems [61] in general relativity even in cases when matter satisfies all energy conditions because quantum geometry effects modify the (geometrical) left hand side of Einstein's equations.

In the classical theory, a FLRW solution corresponds to a trajectory $\nu(t), \phi(t)$, or, eliminating the parameter $t$, just $\nu(\phi)$. A solution $\Psi_{o}(\nu, \phi)$ to (2.7) represents the quantum analog of this trajectory. Since there is no sharp trajectory, we no longer have a single, sharply defined space-time metric in the background. Consequently, we do not have a canonical parameter representing the cosmic or conformal time. At a fundamental level, all dynamics in the Planck regime refers to the relational time $\phi$.

We will be interested in a specific class of states $\Psi_{o}$. To specify that class, it is convenient to first set $V(\phi)=0$ to get a qualitative insight into LQC dynamics. This case has been analyzed in great detail in the LQC literature. One can now start at late times, when the classical approximation is clearly excellent, and consider wave functions which are sharply peaked at a point on the phase space. Under evolution, these states are known to remain sharply peaked and the peaks are known to follow trajectories satisfying certain effective equations that encode the leading-order quantum corrections. These effective solutions are in excellent agreement with the FLRW trajectories of general relativity when the matter density and the curvature are less than a thousandth of the Planck scale [7, 62, 63]. But they strongly deviate from general relativity in the Planck regime: they define singularityfree, bouncing trajectories. This behavior has been established analytically in the $\mathrm{k}=0$, $\Lambda=0$ case [60] and was checked numerically in the $\mathrm{k}=1$, as well as $\Lambda \neq 0$ cases $[27,29,31$. The effective equations themselves are rather general and continue to be meaningful when $V(\phi) \neq 0$, or if we replace the scalar field by other matter sources [64, 65].

In presence of the quadratic potential $V(\phi)=(1 / 2) m^{2} \phi^{2}$, sharply peaked wave functions have been constructed whenever numerical simulations are feasible, which corresponds to the case when the kinetic energy is dominant at the bounce [56]. ${ }^{5}$ Again, the peaks of these wave functions follow solutions to effective equations. We do not see any a priori reason why this general behavior will not continue away from kinetic energy dominated bounces. More importantly, as we will see in sections IV and V, it turns out that states $\Psi_{o}$ which undergo a kinetic energy dominated bounce are the most interesting ones for potentially new physics. Therefore, while our underlying framework is valid for all states, in our detailed analysis and numerical simulations we will restrict ourselves to those solutions $\Psi_{o}(\nu, \phi)$ to (2.7) which are sharply peaked on an effective trajectory from the bounce (at least) until the trajectory enters the regime in which the matter density and curvature are so low that general relativity is an excellent approximation.

Since the FLRW sector of the phase space is a system with a Hamiltonian constraint, we used the Dirac quantization procedure and reinterpreted the constraint as an evolution equation with respect to the internal time $\phi$. This is why we were naturally led to the Schrödinger picture in which states evolve and operators don't. However, in the cosmology literature one generally uses the Heisenberg picture. The transition can be carried out in the usual fashion. In the Heisenberg picture, states are frozen in time and it is the volume operator $\hat{\nu}$ (or scale factor operator $\hat{a}=\left(\kappa \gamma \hbar|\hat{\nu}| / 4 \ell^{3}\right)^{1 / 3}$ ) that evolves with respect to $\phi$ and

\footnotetext{
${ }^{5}$ In this case, it takes about $10^{6}$ Planck seconds to reach the onset of slow roll starting from the bounce (see, e.g., Table 1). As the potential energy at the bounce increases, this period increases rapidly and it has been difficult to maintain the required accuracy for these longer periods.
} 
the space-time metric operator is given by [19]:

$$
\hat{g}_{a b} d x^{a} d x^{b} \equiv d \hat{s}^{2}=\hat{H}_{o}^{-1} \ell^{6} \hat{a}^{6}(\phi) \hat{H}_{o}^{-1} d \phi^{2}+\hat{a}^{2}(\phi) d \vec{x}^{2}
$$

where we have used the fact that, in the classical theory, the lapse corresponding to the scalar field time is $N_{\text {hom }}=a^{3} \ell^{3} / p_{(\phi)}$, and, on physical quantum states, $\hat{p}_{(\phi)} \equiv-i \hbar \partial_{\phi}=\hat{H}_{o}$. The operator $\hat{g}_{a b}$ lives on a 4 -manifold $M$ coordinatized by $\phi, \vec{x}$, where $\phi=$ const surfaces are regarded as 'space' sections. The effective trajectories correspond to using the expectation value $\bar{a}=\left\langle\Psi_{o} \hat{a} \Psi_{o}\right\rangle$ in place of $\hat{a}$ in (2.8).

At a fundamental level, then, the parameter $\phi$ serves as the time variable and the quantum geometry is determined by the physical (Heisenberg) operators $\hat{a}(\phi)$. However, as we will see in the next sub-section, an unforeseen simplification occurs in the dynamics of perturbations even in the Planck regime, enabling us to cast this exact quantum dynamics in terms of the conformal time of a quantum corrected metric.

Remark: Mathematically as well as physically, the deparametrization procedure outlined above is straightforward in the case when the scalar field potential $V(\phi)$ vanishes (for details, see $[7,23,24])$. In the present case one has to incorporate some subtleties on both fronts $[56]$.

On the mathematical side, it turns out that the formal quantum constraint $\hat{\mathbb{S}}_{o}$ again has the operator form $\hbar^{2} \partial_{\phi}^{2}+\Theta$ as in the $V(\phi)=0$ case but now the operator $\Theta$ is no longer essentially self-adjoint [7]. Therefore, to arrive at (2.7), one has to choose a self-adjoint extension of $\Theta$, and $H_{o}$ is then the square-root of the corresponding operator $|\hat{\Theta}|$ which is self-adjoint by construction. This overall situation is the same as in the case when $V(\phi)=0$ but there is a positive cosmological constant $\Lambda$. That case is well-understood [31] and, for states of physical interest, the results have been shown to be quite insensitive of the choice of the self-adjoint extension. In the present case, the existing results indicate that the situation should be similar.

On the physical side, in the $V(\phi)=0$ case $\phi$ serves as a global time parameter along classical dynamical trajectories. This is no longer the case when $V(\phi) \neq 0$. Consequently, one has to work with 'patches' of dynamical trajectories such that $\phi$ is monotonic in each patch. In simple examples, the associated subtleties in the quantum theory have been discussed in the literature (see, e.g., [66, 67]). But for our purposes, the situation is simpler because we can work just in one 'patch'. This is because we will start at the bounce with $\phi$ increasing and by the time it 'turns around' after climbing up the potential, the energy density and curvature are so low compared to the Planck scale that general relativity is a good approximation. Thus, $\phi$ is in fact single valued in the regime in which full quantum treatment of the background geometry is needed.

\section{Effective equations}

The effective equations of LQC, which track the evolution of the peaks of wave functions $\Psi_{o}$, have a number of consequences that seem surprising if one's intuition is based largely on inflationary dynamics within general relativity. In this sub-section we will summarize these features. While our focus is on the quadratic potential, most of these results hold for general (regular) potentials. 
- The Hubble parameter: Recall that on solutions to Einstein's equations, the momentum b conjugate to $\nu$ is related to the standard Hubble parameter $H=\dot{a} / a$ via $b=\gamma H$. However, on solutions to the LQC effective equations we have

$$
H=\frac{1}{2 \gamma \lambda} \sin 2 \lambda \mathrm{b} \approx(0.93) \sin 2 \lambda \mathrm{b},
$$

where $\lambda^{2}=4 \sqrt{3} \pi \gamma \ell_{\mathrm{Pl}}^{2} \approx 5.2$ is the 'area-gap' that sets the discreteness scale of LQC. Thus, in striking contrast to general relativity, the Hubble parameter $H$ is bounded in $L Q C$ by 0.93 in Planck units. b ranges over $(0, \pi / \lambda)$; where $\mathrm{b}=\pi / \lambda$ corresponds to the infinite past and $\mathrm{b}=0$ to the infinite future. General relativity is recovered in the limit $\lambda \rightarrow 0$, i.e., when quantum geometry effects can be ignored. In this limit, $b$ ranges over $(0, \infty)$ and $b=\infty$ corresponds to the big-bang. Finally, note that while $H$ is monotonic in general relativity (in the absence of spatial curvature and provided $\rho>0$ ), (2.9) implies that this is not the case in LQC.

- The Friedmann equation: Quantum geometry corrections modify the left side of Einstein's equations. In particular, the Friedmann equation becomes

$$
\frac{\sin ^{2} \lambda \mathrm{b}}{\gamma^{2} \lambda^{2}}=\frac{8 \pi G}{3} \rho \equiv \frac{8 \pi G}{3}\left(\frac{\dot{\phi}^{2}}{2}+V(\phi)\right)
$$

To compare with the standard Friedmann equation $H^{2}=(8 \pi G / 3) \rho$, it is often convenient to write (2.10) using (2.9):

$$
\frac{1}{9}\left(\frac{\dot{\nu}}{\nu}\right)^{2} \equiv H^{2}=\frac{8 \pi G}{3} \rho\left(1-\frac{\rho}{\rho_{\max }}\right)
$$

where $\rho_{\max }=3 / 8 \pi G \gamma^{2} \lambda^{2} \approx 0.41 \rho_{\mathrm{Pl}}$. Thus the familiar linear relation between the Hubble parameter and the matter density no longer holds. Nonetheless, as is obvious from Eqs (2.9) - (2.11), away from the Planck regime - i.e., when $\lambda b \ll 1$, or, $\rho \ll \rho_{\max }$ - we recover classical general relativity.

- Bounces: In general relativity, the Friedmann equation implies that, if the matter density is positive, $\dot{a}$ cannot vanish (in the absence of curvature or a cosmological constant). Consequently, in any given solution, the universe is either contracting, or an expanding. By contrast, (2.11) now implies that $\dot{\nu}$ vanishes at $\rho=\rho_{\max }$; this is the quantum bounce. To its past, the solution represents a contracting universe with $\dot{\nu}<0$ and to its future, an expanding one with $\dot{\nu}>0$.

In LQC, b is monotonically non-increasing (if we ignore the exceptional de Sitter solutions that exist for certain potentials), evolving from $b=\pi / \lambda$ in the infinite past to 0 in the infinite future. Eqs (2.10) and (2.11) imply that $\mathrm{b}=\pi / 2 \lambda$ at the bounce. Thus, each solution undergoes precisely one bounce.

- Boundedness of physical quantities: Intuitively one can think of the big bounce of LQC as the replacement of the big bang in general relativity. However, all physical quantities remain bounded at the big bounce.

As we have already noted, the matter density achieves its maximum value $\rho_{\max }$ at the bounce. When the potential $V(\phi)$ is bounded below, the Ricci scalar - the only non-trivial curvature scalar in these models - is bounded above by $\approx 62$ and $|\dot{H}|$ is bounded above by $\approx 10.29$. If the potential $V(\phi)$ is bounded below, say $V \geq V_{o}$, then it follows from $(2.10)$ that $\dot{\phi}^{2}$ is bounded by $2 \rho_{\max }-2 V_{o}$. If $V$ grows unboundedly for large $|\phi|$, then $|\phi|$ is also 
bounded. For example, for $V=m^{2} \phi^{2} / 2$, we have $m|\phi|_{\max } \approx 0.90$. Finally, one can also show that if $\nu \neq 0$ initially, it cannot vanish in finite proper time along any solution.

Perhaps the most striking contrast with general relativity occurs in the behavior of the Hubble parameter $H$ : It vanishes at the bounce while in general relativity it diverges at the singularity and is large throughout the Planck regime. As one would expect, $H$ is bounded above in LQC, $|H| \lesssim 0.93$, and achieves its upper bound in every solution at the end of super-inflation $(\mathrm{b}=\pi / 4 \lambda)$ that follows immediately after the bounce.

To summarize, solutions to the effective equations in LQC are everywhere regular irrespective of whether one focuses on matter density, curvature or the scale factor.

\section{QFT on quantum FLRW space-times}

In striking contrast to the FLRW solutions to Einstein's equations, the quantum geometry $\Psi_{o}$ is regular; there is no big bang singularity. The quantum perturbations $\hat{\mathcal{Q}}_{\vec{k}}, \hat{\mathcal{T}}_{\vec{k}}$ propagate on this regular quantum geometry. Therefore this framework is well suited to incorporate Planck scale physics by facing various quantum gravity issues directly. Our task is to specify the dynamics of quantum fields $\hat{\mathcal{Q}}_{\vec{k}}, \hat{\mathcal{T}}_{\vec{k}}$ on any given quantum background $\Psi_{o}(\nu, \phi)$. At first this task seems formidable. However, the detailed framework of QFT on quantum FLRW space-times introduced in [48] and further developed in [19] shows that there is an unforeseen simplification.

\section{Dressed, effective metric}

Let us start with the tensor perturbations. In the classical theory, the dynamics of $\mathcal{T}_{\vec{k}}$ is the same as that of a zero rest mass scalar field on the background FLRW metric. In the quantum theory, we have the following surprising result.

- Within the test field approximation inherent to our truncation strategy, the dynamics of $\hat{\mathcal{T}}_{\vec{k}}$ on any one of our background quantum geometries $\Psi_{o}$ is completely equivalent to that of the quantum field $\hat{\mathcal{T}}_{\vec{k}}$ propagating on a smooth but quantum corrected metric $\tilde{g}_{a b}$ given by

$$
\tilde{g}_{a b} d x^{a} d x^{b} \equiv d \tilde{s}^{2}=-\left(\tilde{p}_{(\phi)}\right)^{-2} \ell^{6} \tilde{a}^{6}(\phi) d \phi^{2}+\tilde{a}^{2}(\phi) d \vec{x}^{2}
$$

where

$$
\left(\tilde{p}_{(\phi)}\right)^{-1}=\left\langle\hat{H}_{o}^{-1}\right\rangle \quad \text { and } \quad \tilde{a}^{4}=\frac{\left\langle\hat{H}_{o}^{-\frac{1}{2}} \hat{a}^{4}(\phi) \hat{H}_{o}^{-\frac{1}{2}}\right\rangle}{\left\langle\hat{H}_{o}^{-1}\right\rangle} .
$$

Here the expectation value is taken in the quantum geometry state $\Psi_{o}$ and $\hat{a}(\phi)$ is the (Heisenberg) operator on $\mathcal{H}_{o}$ describing the scale factor. This is an exact mathematical equivalence.

Let us now turn to the scalar perturbations. In the classical theory, the dynamics of $\mathcal{Q}_{\vec{k}}$ is governed not only by the background FLRW metric $g_{a b}$ but also by a time dependent external potential $\mathfrak{A}$ (see 2.5 ). In the quantum theory we have: 
- Again, within the test field approximation, the dynamics of $\hat{\mathcal{Q}}_{\vec{k}}$ on any one of our background quantum geometries $\Psi_{o}$ is completely equivalent to that of the quantum field $\hat{\mathcal{Q}}_{\vec{k}}$ propagating on a smooth but quantum corrected geometry $\tilde{g}_{a b}$ given by $(2.12)$ and a quantum corrected time-dependent potential $\tilde{\mathfrak{A}}$, given by

$$
\tilde{\mathfrak{A}}(\phi)=\frac{\left\langle\hat{H}_{o}^{-\frac{1}{2}} \hat{a}^{2}(\phi) \hat{\mathfrak{A}}(\phi) \hat{a}^{2}(\phi) \hat{H}_{o}^{-\frac{1}{2}}\right\rangle}{\left\langle\hat{H}_{o}^{-\frac{1}{2}} \hat{a}^{4}(\phi) \hat{H}_{o}^{-\frac{1}{2}}\right\rangle} .
$$

For tensor modes, this result was derived systematically in [19] and the generalization to the scalar modes follows the same steps. The essence of the result is that while the quantum perturbations $\hat{\mathcal{T}}(\vec{x}, \phi)$ and $\hat{\mathcal{Q}}(\vec{x}, \phi)$ propagate on the quantum geometry of $\Psi_{o}$, they do not experience all the details of quantum fluctuations encoded in this state. The net effect of the quantum geometry on their dynamics is neatly encoded in just three time-dependent quantities constructed from $\Psi_{o}: \tilde{p}_{(\phi)}, \tilde{a}, \tilde{\mathfrak{A}}$. This is similar to what happens when light propagates in a medium. While it interacts with the atoms of the medium, the net effect can be encoded in just a few parameters such as the refractive index. Quantum geometry can be thought of as the medium through which the quantum fields $\hat{\mathcal{Q}}, \hat{\mathcal{T}}$ propagate and the net effect of the medium is to provide quantum corrections (2.13) and (2.14) to $\eta, a$ and $\mathfrak{A}$.

Next, note that in this exact result the effective quantities that enter are not just expectation values of the corresponding operators; they are much more sophisticated and, in particular, they are sensitive also the quantum fluctuations in $\Psi_{o}$. Indeed their definitions are quite intricate and could not have been arrived at without a detailed calculation. In particular, in the classical theory $p_{(\phi)}(\phi)$ and $a(\phi)$ not only determine the metric $g_{a b}$ but also the external, time dependent potential $\mathfrak{A}$ experienced by the scalar perturbation $\mathcal{Q}_{\vec{k}}$. In the quantum corrected description, by contrast, the potential $\tilde{\mathfrak{A}}$ is not determined by $\tilde{p}_{(\phi)}$ and $\tilde{a}$; it has to be calculated independently. Conceptually this is an important implication of the underlying quantum geometry.

We will refer to $\tilde{g}_{a b}$ as the dressed, effective geometry to distinguish it from the metric $\bar{g}_{a b}$ defined by the effective trajectories, discussed in section II B, that track just the peak of the wave function $\Psi_{o}$. Similarly, we will refer to $\tilde{\mathfrak{A}}$ as the dressed, effective, external potential. The result on exact equivalence of dynamics holds only for the dressed effective metric $\tilde{g}_{a b}$. However, in practice, from the bounce until the effective trajectory enters the general relativistic regime, the wave function $\Psi_{o}$ is so sharply peaked on the effective trajectory that the difference between $\bar{g}_{a b}$ and $\tilde{g}_{a b}$ would be too small to track accurately in numerical simulations (since even in sophisticated simulations the numerical errors are much larger). Therefore in numerical simulations in section V we will not distinguish between $\tilde{g}_{a b}$ and $\bar{g}_{a b}$.

Finally, although its coefficients (in the $\phi, \vec{x}$ chart) depend on $\hbar, \tilde{g}_{a b}$ is a smooth tensor field. Therefore, it is now straightforward to pass to the conformal time $\tilde{\eta}$ it defines. We have:

$$
\tilde{g}_{a b} d x^{a} d x^{b} \equiv d \tilde{s}^{2}=\tilde{a}\left(-d \tilde{\eta}^{2}+d \vec{x}^{2}\right),
$$

where

$$
d \tilde{\eta}=\left[\ell^{3} \tilde{a}^{2}\right] \tilde{p}_{(\phi)}^{-1} d \phi .
$$

This description is especially convenient in the general relativistic regime to make direct contact with the cosmology literature.

To summarize, the dynamics of quantum fields $\hat{\mathcal{T}}_{\vec{k}}$ on a quantum geometry $\Psi_{o}$ is mathematically the same as that of their dynamics on curved background geometry (2.15). For 
the scalar mode $\hat{\mathcal{Q}}_{\vec{k}}$, the classical 'external potential' $\mathfrak{A}$ has also to be replaced by a quantum corrected external potential given by (2.14). This equivalence greatly simplifies our task of defining the appropriate Hilbert space and operators for $\hat{\mathcal{Q}}_{\vec{k}}, \hat{\mathcal{T}}_{\vec{k}}$ because we can import into quantum field theory on quantum FLRW space-times of [48] the rich set of techniques that have been developed in the QFT on classical FLRW space-times. We will summarize the main results in section II C 2 . However, we emphasize that the equivalence holds only if the perturbations can be regarded as test fields; i.e., their back reaction can be neglected. For now we will assume that this is the case. At the end, in section VI, we will carry out a self-consistency test by verifying that the assumption is in fact met.

\section{Hilbert space $\mathcal{H}_{1}$ of perturbations and operators thereon}

In this subsection we briefly summarize the characterization of the physical Hilbert $\mathcal{H}_{1}$ of perturbations and the appropriate regularization of relevant composite operators, such as energy density. We will work with the adiabatic regularization approach [68, 69], which is particularly convenient to perform explicit computations in the cosmological context. For simplicity of presentation we will first discuss the tensor modes and then summarize the modifications needed to incorporate the scalar mode.

We can follow the standard procedure used in the cosmology literature since the perturbations can be regarded as propagating on a FLRW metric $\tilde{g}_{a b} .{ }^{6}$ The symmetries of this dressed, effective background $\tilde{g}_{a b}$ allow us to expand the field operator $\hat{\mathcal{T}}(\vec{x}, \tilde{\eta})$ in Fourier modes

$$
\hat{\mathcal{T}}(\vec{x}, \tilde{\eta})=\int \frac{d^{3} k}{(2 \pi)^{3}}\left(\hat{A}_{\vec{k}} e_{k}(\tilde{\eta})+\hat{A}_{-\vec{k}}^{\dagger} e_{k}^{\star}(\tilde{\eta})\right) e^{i \vec{k} \cdot \vec{x}}
$$

where, as usual, $k=|\vec{k}| .{ }^{7}$ The operators $\hat{\mathcal{T}}(\vec{x}, \tilde{\eta})$ satisfy the Heisenberg equation of motion if and only if the mode functions $e_{k}(\tilde{\eta})$ are solutions of the wave equation

$$
e_{k}^{\prime \prime}(\tilde{\eta})+2 \frac{\tilde{a}^{\prime}}{\tilde{a}} e_{k}^{\prime}(\tilde{\eta})+k^{2} e_{k}(\tilde{\eta})=0
$$

were the 'prime' denotes the derivative with respect to $\tilde{\eta}$. These solutions $e_{k}(\tilde{\eta})$ are to provide a generalization of the positive frequency basis $e^{-i k t} / \sqrt{2 k}$ used in Minkowski spacetime. The canonical commutation relations for the field operator $\hat{\mathcal{T}}(\vec{x}, \tilde{\eta})$ and its conjugate momentum imply

$$
\left[\hat{A}_{\vec{k}}, \hat{A}_{\vec{k}^{\prime}}^{\dagger}\right]=i \hbar(2 \pi)^{3} \delta\left(\vec{k}-\vec{k}^{\prime}\right)\left\langle e_{k}(\tilde{\eta}), e_{k^{\prime}}(\tilde{\eta})\right\rangle^{-1} \quad \text { and } \quad\left[\hat{A}_{\vec{k}}, \hat{A}_{\vec{k}^{\prime}}\right]=0
$$

where

$$
\left\langle e_{k}(\tilde{\eta}), e_{k^{\prime}}(\tilde{\eta})\right\rangle:=\frac{\tilde{a}^{2}}{4 \kappa}\left(e_{k}(\tilde{\eta}) e_{k^{\prime}}^{\star \prime}(\tilde{\eta})-e_{k}^{\prime}(\tilde{\eta}) e_{k^{\prime}}^{\star}(\tilde{\eta})\right)
$$

Therefore, if we require that the basis functions $e_{k}(\tilde{\eta})$ in $(2.17)$ to satisfy the normalization condition $\left\langle e_{k}(\tilde{\eta}), e_{k}(\tilde{\eta})\right\rangle=i$, then $\hat{A}_{\vec{k}}$ and $\hat{A}_{\vec{k}}^{\dagger}$ satisfy the familiar commutation relations of

\footnotetext{
${ }^{6}$ For a more mathematical treatment involving representations of Weyl algebras, see [19].

${ }^{7}$ Since $\mathcal{T}(\vec{x}, \tilde{\eta})$ is purely inhomogeneous in the classical theory, it is natural to require the same property for $\hat{\mathcal{T}}(\vec{x}, \tilde{\eta})$. Then, the integral excludes the point $k=0$. But we will require that $e_{k}(\tilde{\eta})$ is continuous in $k$ and $k e_{k}(\tilde{\eta})$ admits a limit as $k \rightarrow 0$.
} 
creation and annihilation operators. Note that, because $e_{k}(\tilde{\eta})$ and $e_{k^{\prime}}(\tilde{\eta})$ are solutions of (2.18), the scalar product $\left\langle e_{k}(\tilde{\eta}), e_{k^{\prime}}(\tilde{\eta})\right\rangle$ is constant in time. Therefore it suffices to impose it at some initial instant.

The first steps in the construction of the Hilbert space $\mathcal{H}_{1}$ of tensor perturbations can be summarized as follows: i) Choose a family of normalized solutions $e_{k}(\tilde{\eta})$ to $(2.18)$; ii) Define the associated vacuum state $|0\rangle$ as the state annihilated by all $\hat{A}_{\vec{k}}$; and, iii) Construct the Fock space generated by a repeated action of creation operators $\hat{A}_{\vec{k}}^{\dagger}$ on the vacuum. With further important qualifications discussed below, this Fock space will be the required $\mathcal{H}_{1}$.

The vacuum state constructed in this way is invariant under the translational and rotational isometries of $\tilde{g}_{a b}$. This property will be will be important in our later discussion and follows from the symmetries of the 2-point function

$$
\left\langle 0\left|\hat{\mathcal{T}}\left(\vec{x}_{1}, \tilde{\eta}_{1}\right) \hat{\mathcal{T}}\left(\vec{x}_{2}, \tilde{\eta}_{2}\right)\right| 0\right\rangle=\hbar \int \frac{d^{3} k}{(2 \pi)^{3}}\left[e^{i \vec{k} \cdot\left(\vec{x}_{1}-\vec{x}_{2}\right)} e_{k}\left(\tilde{\eta}_{1}\right) e_{k}^{\star}\left(\tilde{\eta}_{2}\right)\right]
$$

which suffices to completely characterize this Fock representation. However, it is clear from the above construction that the vacuum state is far from being unique: Different choices for the bases $e_{k}$ lead to different definitions of operators $\hat{A}_{\vec{k}}$ and $\hat{A}_{\vec{k}}^{\dagger}$, and therefore different vacua. In the absence of additional physical inputs - such as invariance under the much larger isometry groups available in Minkowski or de Sitter space-times - one cannot single out a preferred vacuum. More importantly, there is no a priori guarantee that the vacuum chosen by a basis $e_{k}$ would belong to the Fock space determined by a different basis $\underline{\mathrm{e}}_{k}$. There are two sets of potential problems: ultra-violet and infra-red.

In cosmological backgrounds the ultra-violet issues can be faced by imposing appropriate regularity condition on the basis $e_{k}$ that select the Fock space. In the FLRW backgrounds, one can impose the adiabatic condition [68-70], i.e. require that the $e_{k}$ must approach the Minkowski space-time positive frequency modes, $e^{-i k t} / \sqrt{2 k}$, at an appropriate rate in the limit in which the physical momentum $k / \tilde{a}$ is much larger than the energy scale $E_{\text {curv }}$ provided by the space-time curvature. (For a succinct discussion of the technical statements, see the companion paper [19].) The rate of approach determines the adiabatic order of the basis $e_{k}$ and controls the ultraviolet behavior of states in the Fock space it selects. Since the stress-energy tensor is a dimension 4 operator, for it to be well-defined one needs the basis $e_{k}$ to be of 4 th adiabatic order. We impose this restriction.

Had the spatial hypersurfaces been compact — with, say, $\mathbb{T}^{3}$ topology rather than $\mathbb{R}^{3}$ the Fock representations arising from any two 4 th order adiabatic bases $e_{k}$ and $\underline{\mathrm{e}}_{k}$ would have been unitarily equivalent. In particular, all the 4 th order adiabatic vacua would lie in the same Hilbert space, which we could take as our $\mathcal{H}_{1}$. However even in this case there would be no 'preferred' vacuum state: each regular basis would define one and, without additional physical input, there is a no preferred basis. However, the total number of particles $\langle 0|\underline{\hat{N}}| 0\rangle$ defined by (the creation and annihilation operators associated with) a regular basis $\underline{\mathrm{e}}_{k}$ in the vacuum state $|0\rangle$ defined by another regular basis $e_{k}$ would be finite.

In this paper, the spatial topology is $\mathbb{R}^{3}$ and there is a further, infrared difficulty. Now, the adiabatic regularity only ensures that the spatial number density of the 'under-barred' particles in the vacuum $|0\rangle$ is finite. Furthermore, since each vacuum is translationally invariant, this number density is constant in space. Therefore, the total number is infinite for the trivial reason that the spatial volume is infinite! This infrared infinity is generally regarded as physically spurious. However, mathematically it implies that, generically, the vacuum $|\underline{0}\rangle$ defined by a regular basis $\underline{\mathrm{e}}_{k}$ would have infinite norm in the Fock space defined 
by another regular basis $e_{k}$, whence the two Fock representations are unitarily inequivalent. However, they can be regarded as physically equivalent in the sense that the expectation values of any 'under-barred' observable that refers to a compact region is well-defined in $|0\rangle$ (and hence on a dense subspace of the corresponding Fock space). This region could be $\mathbb{R}_{\mathrm{LS}}$, the portion of the (infinite) surface of last scattering that is accessible to observations (physically, $\mathbb{R}_{\mathrm{LS}}$ is intertwined with the background quantum geometry $\Psi_{o}$ ). For example, for the number operator $\hat{N}\left(\mathbb{R}_{\mathrm{LS}}\right)$, corresponding to the number of 'under-barred particles' in the spatial region $\mathfrak{R}_{\mathrm{LS}},\left\langle 0\left|\underline{\hat{\mathrm{N}}}\left(\mathfrak{R}_{\mathrm{LS}}\right)\right| 0\right\rangle$ would be finite for any regular bases $e_{k}$ and $\underline{\mathrm{e}}_{k}$.

Thus, in the $\mathbb{R}^{3}$ topology under consideration, for definiteness, we will fix a basis $e_{k}$ which is regular up to the 4 th adiabatic order and take $\mathcal{H}_{1}$ to be the Fock space it defines (specific examples of convenient bases will be discussed in section IV). All operators of physical interest to us will be well-defined on $\mathcal{H}_{1}$. Furthermore, observables such as energy density, or particle number and energy in a compact region, so constructed, will have well-defined expectation values on Fock spaces constructed from any other basis $\underline{\mathrm{e}}_{k}$ which is also regular up to 4 th adiabatic order.

The expansion $(2.17)$ of $\hat{\mathcal{T}}(\vec{x}, \tilde{\eta})$ immediately implies that it is a well-defined operator valued distribution on $\mathcal{H}_{1}$, i.e. that $\int d^{3} x \hat{\mathcal{T}}(\vec{x}, \tilde{\eta}) f(\vec{x})$ is a well-defined (self-adjoint) operator on $\mathcal{H}_{1}$ for every smooth function $f(\vec{x})$ of compact support. However, since observables such as energy density involve a product of these operator valued distributions, they have to be regularized. It is here that the 4th order adiabatic regularity plays a crucial role. It provides a natural, mode by mode subtraction scheme that removes the ultra-violet divergences in a local and state independent manner, while respecting the covariance of the underlying theory.

Let us begin with the energy density operator for tensor modes. Recall first that in the classical theory the stress-energy tensor is given by

$$
T_{a b}=\frac{1}{4 \kappa}\left[\tilde{\nabla}_{a} \mathcal{T} \tilde{\nabla}_{b} \mathcal{T}-\frac{1}{2} \tilde{g}_{a b} \tilde{g}^{c d} \tilde{\nabla}_{c} \mathcal{T} \tilde{\nabla}_{d} \mathcal{T}\right]
$$

Therefore, (2.17) implies that the expectation value of the energy density operator $\hat{\rho}^{(\mathcal{T})}(\vec{x}, \tilde{\eta})$ in the vacuum state associated with a regular basis $e_{k}$ is formally given by

$$
\left\langle 0\left|\hat{\rho}^{(\mathcal{T})}\right| 0\right\rangle_{\text {formal }}:=\left\langle 0\left|\hat{T}_{a b} \tilde{t}^{a} \tilde{t}^{b}\right| 0\right\rangle_{\text {formal }}=\frac{\hbar}{8 \kappa \tilde{a}^{2}} \int \frac{d^{3} k}{(2 \pi)^{3}}\left[\left|e_{k}^{\prime}\right|^{2}+k^{2}\left|e_{k}\right|^{2}\right],
$$

where $\tilde{t}^{a}$ is the unit normal to the homogeneous slices w.r.t. $\tilde{g}_{a b}$. The formal expression is ultraviolet divergent. The adiabatic regularization scheme provides a specific mode by mode substraction, yielding

$$
\left\langle 0\left|\hat{\rho}^{(\mathcal{T})}(\vec{x}, \tilde{\eta})\right| 0\right\rangle=\frac{\hbar}{8 \kappa \tilde{a}^{2}} \int \frac{d^{3} k}{(2 \pi)^{3}}\left[\left|e_{k}^{\prime}\right|^{2}+k^{2}\left|e_{k}\right|^{2}-4 \kappa C^{(\mathcal{T})}(k, \tilde{\eta})\right],
$$

where the subtraction term $C^{(\mathcal{T})}(k, \tilde{\eta})$ is given by

$$
C^{(\mathcal{T})}(k, \tilde{\eta})=\frac{k}{\tilde{a}^{2}}+\frac{\tilde{a}^{\prime 2}}{2 \tilde{a}^{4} k}+\frac{4 \tilde{a}^{\prime 2} \tilde{a}^{\prime \prime}+\tilde{a} \tilde{a}^{\prime \prime 2}-2 \tilde{a} \tilde{a}^{\prime} \tilde{a}^{\prime \prime \prime}}{8 \tilde{a}^{5} k^{3}} .
$$

This subtraction tames the ultraviolet divergences. But there is a further subtlety, again because the spatial topology is $\mathbb{R}^{3}$ (rather than $\mathbb{T}^{3}$ ) and $\mathcal{T}(\vec{x}, \tilde{\eta})$ is effectively a zero rest 
mass field: the right side of (2.24) has an infrared divergence, directly inherited from the $1 / k^{3}$ term in $C^{(\mathcal{T})}(k, \tilde{\eta})$. Therefore, we need to introduce an infrared cut-off. A natural strategy to handle this issue is to absorb modes with wavelengths larger than the radius $\mathfrak{R}(\tilde{\eta})$ of observable universe into the definition of the homogeneous background itself. Given an instant of time $\tilde{\eta}$, this provides us with a minimum physical frequency (or maximum physical wavelength) for the modes that are to be treated as arising from perturbations. Thus, we are led to the following strategy: Perturbations whose physical wavelength is shorter than $\mathfrak{R}(\tilde{\eta})$ will contribute to $\left\langle 0^{\text {obv }}|\hat{\rho}| 0^{\text {obv }}\right\rangle_{\text {ren }}$ while those with larger wave lengths will be treated as contributing to the background. This procedure is supported by the fact that, if we were to use a 3-torus spatial topology, the current observational limits on the radius of the torus is close to $\mathfrak{R}(\tilde{t})$ [71]. Therefore, had we used a torus with this radius, the infrared cut-off we use would have been implemented automatically.

Note that, in terms of co-moving wave numbers $k$ used in our analysis, this infrared cut-off is in fact time-independent: It is given by $k_{o}=k_{\star} / 8.58$ where $k_{\star}$ is the reference mode used by the WMAP (see section III A below). As discussed in section VI B of [19], an infrared cut-off with fixed co-moving $k_{\text {min }}$ leads to a renormalized energy density satisfying various criteria that are generally used to select viable renormalization procedures in QFT in curved space-times. Thus, the strategy is consistent with the conceptual framework of the renormalization theory.

The final expression of the expectation value of the renormalized energy density operator is given by

$$
\left\langle 0\left|\hat{\rho}^{(\mathcal{T})}(\vec{x}, \tilde{\eta})\right| 0\right\rangle_{\mathrm{ren}}=\frac{\hbar}{8 \kappa \tilde{a}^{2}} \int_{k_{\min }}^{\infty} \frac{d^{3} k}{(2 \pi)^{3}}\left[\left|e_{k}^{\prime}\right|^{2}+k^{2}\left|e_{k}\right|^{2}-4 \kappa C^{(\mathcal{T})}(k, \tilde{\eta})\right] .
$$

where $C^{(\mathcal{T})}(k, \tilde{\eta})$ is again given by $(2.25)$. Note that the right hand side is independent of $\vec{x}$ because the vacuum is translationally invariant. The same procedure yields the matrix elements of $\hat{\rho}_{\text {ren }}$ on the obvious dense subspace of $\mathcal{H}_{1}$ obtained by operating on the vacuum by finite linear combinations of products of creation operators. These of course depend on both $\vec{x}$ and $\tilde{\eta}$. The matrix elements of the entire stress-energy operator can be constructed in the same fashion. We have focused on energy density because, as noted below, it is the integrand of the Hamiltonian operator; its expectation values determine whether our truncation approximation is valid [19].

Remark: As shown in [19], the Hamiltonian operator that governs the evolution of $\hat{\mathcal{T}}_{\vec{k}}$ in conformal time $\tilde{\eta}$ has the formal expression:

$$
\hat{H}_{1, \text { formal }}^{(\mathcal{T})}=\int \frac{d^{3} k}{(2 \pi)^{3}}\left(\frac{2 \kappa}{\tilde{a}^{2}}\left|\hat{\mathfrak{p}}_{\vec{k}}^{(\mathcal{T})}\right|^{2}+\frac{\tilde{a}^{2} k^{2}}{8 \kappa}\left|\hat{\mathcal{T}}_{\vec{k}}\right|^{2}\right)
$$

(In light of (2.4), this expression can be anticipated once we know the equivalence between quantum fields $\hat{\mathcal{T}}$ propagating on the quantum geometry $\Psi_{o}$ and $\tilde{g}_{a b}$.) Now, the standard relation between the energy density and the Hamiltonian generating evolution in the conformal time implies $\hat{H}_{1, \text { formal }}^{(\mathcal{T})}=\tilde{a}^{4} \int d^{3} x \hat{\rho}_{\text {formal }}^{(\mathcal{T})}$. Therefore, even if we replace $\hat{\rho}_{\text {formal }}^{(\mathcal{T})}$ with $\hat{\rho}_{\text {ren }}^{(\mathcal{T})}$ with its infrared cut-off $k_{\text {min }}$, the vacuum expectation value of the Hamiltonian operator still has the trivial divergence simply because the integrand is constant and the total volume is infinite. However, as discussed above, we can restrict the space integral just to the spatial region $\mathbb{R}_{\text {obs }}(\tilde{\eta})$ that is observable at time $\tilde{\eta}$ and obtain a Hamiltonian 
operator $\hat{H}_{\text {obs }}(\tilde{\eta})$ tailored to $\mathbb{R}_{\text {obs }}(\tilde{\eta})$. It provides the desired evolution of operators smeared with (space-time) test functions with support anywhere in the observable universe. (For a more mathematically complete discussion, see [19].)

Finally, let us consider the scalar perturbations $\hat{\mathcal{Q}}$. Conceptually the situation is parallel. The mode functions $q_{k}$ (analogous to the $e_{k}$ for tensor perturbations) now satisfy the equation

$$
q_{k}^{\prime \prime}(\tilde{\eta})+2 \frac{\tilde{a}^{\prime \prime}}{\tilde{a}} q_{k}^{\prime}(\tilde{\eta})+\left(k^{2}+\tilde{\mathfrak{A}}\right) q_{k}(\tilde{\eta})=0 .
$$

The energy density operator (that is needed to test the validity of the truncation approximation [19]) is given by:

$$
\left\langle 0\left|\hat{\rho}^{(\mathcal{Q})}(\vec{x}, \tilde{\eta})\right| 0\right\rangle_{\mathrm{ren}}=\frac{\hbar}{2 \tilde{a}^{2}} \int_{k_{\min }}^{\infty} \frac{d^{3} k}{(2 \pi)^{3}}\left[\left|q_{k}^{\prime}\right|^{2}+\left(\tilde{\mathfrak{A}}+k^{2}\right)\left|q_{k}\right|^{2}-C^{(\mathcal{Q})}(k, \tilde{\eta})\right],
$$

where the subtraction term $C^{(\mathcal{Q})}(k, \tilde{\eta})$ is now given by

$$
C^{(\mathcal{Q})}(k, \tilde{\eta})=\frac{k}{\tilde{a}^{2}}+\frac{\tilde{a}^{\prime 2}+\tilde{a}^{2} \tilde{\mathfrak{A}}}{2 \tilde{a}^{4} k}+\frac{-\tilde{a}^{3} \tilde{\mathfrak{A}}^{2}+2 \tilde{a}^{2} \tilde{a}^{\prime} \tilde{\mathfrak{A}}^{\prime}+4 \tilde{a}^{\prime 2} \tilde{a}^{\prime \prime}+\tilde{a}\left(\tilde{a}^{\prime \prime 2}-2 \tilde{a}^{\prime}\left(\tilde{\mathfrak{A}} \tilde{a}^{\prime}+\tilde{a}^{\prime \prime \prime}\right)\right)}{8 \tilde{a}^{5} k^{3}} .
$$

In summary, we have lifted the adiabatic techniques from quantum field theory in curved space-times to quantum fields $\hat{\mathcal{T}}$ and $\hat{\mathcal{Q}}$ propagating on FLRW quantum geometries $\Psi_{o}$. These techniques led us to quantum states of perturbations that have a good behavior in the ultraviolet and to a procedure to systematically regularize products of operator valued distributions that are of direct physical interest. Because of $\mathbb{R}^{3}$ topology, there are some subtleties associated with infrared divergences. However, they can be handled by restricting attention to spatially compact regions of direct physical interest.

\section{INITIAL CONDITIONS}

Now that we have well-defined Hilbert spaces $\mathcal{H}_{o}$ and $\mathcal{H}_{1}$ and physical operators thereon, given an initial state on $\mathcal{H}_{o} \otimes \mathcal{H}_{1}$, we can evolve it all the way through the inflationary era and calculate power spectra and spectral indices. Our primary goal is to explore if there exist viable candidates of initial states that lead to: i) a slow roll inflationary phase that is compatible with the 7 year WMAP data, ii) predictions for power spectra and spectral indices that are compatible with current observations, and, iii) deviations from the $\mathrm{BD}$ vacuum at the onset of inflation. If the answer to the first two questions is in the affirmative, we will have a viable extension of the inflationary scenario to the Planck regime. If the answer to the third question is also in the affirmative, we will have means to test LQC signatures of pre-inflationary dynamics for future observations. Since the bounce replaces the big bang in LQC we will specify the initial state $\Psi_{o} \otimes \psi$ at the bounce.

The emphasis in this paper is on investigating the existence of such initial conditions rather than on their uniqueness. Nonetheless, at the end, we will provide strong motivation for our choices using symmetries, regularity and the novel 'repulsive force' in the Planck regime of LQC, created by the underlying quantum geometry. We hope that future investigations will develop these considerations into a more systematic procedure to arrive at our initial conditions from first principles. 


\section{A. Initial conditions for the background quantum state $\Psi_{o}$}

As explained in section II B, in this paper we focus on quantum states $\Psi_{o}$ of the background geometry which are sharply peaked on effective trajectories of LQC. Therefore, to specify initial conditions for $\Psi_{o}$ we have to examine the effective solutions in some detail. Our task is two-fold: First isolate the initial data at the bounce for effective solutions, and then single out the portion $\mathfrak{P}$ of the permissible initial data set that yields trajectories compatible with the 7 year WMAP data.

Let us begin by noting that in the context of inflationary models, the seven year WMAP data [72] is generally parameterized assuming that the state of perturbations at the onset of inflation is the BD vacuum. In this parametrization, a combination of theory and observations fixes the inflaton mass to be [6]

$$
m=1.21 \times 10^{-6} .
$$

It also implies that the reference co-moving mode $k_{\star}$ used by WMAP ${ }^{8}$ exits the Hubble radius during slow roll at a time $t\left(k_{\star}\right)$ at which the Hubble parameter $H$, the slow roll parameter $\epsilon=-\dot{H} / H^{2}$, the inflaton $\phi$, and its time derivatives $\dot{\phi}$ have the following values [6]:

$$
\begin{array}{rll}
H\left(t\left(k_{\star}\right)\right)=7.83 \times 10^{-6} ; & \text { and } & \epsilon\left(t\left(k_{\star}\right)\right)=8 \times 10^{-3} \\
\phi\left(t\left(k_{\star}\right)\right)= \pm 3.15 & \text { and } & \dot{\phi}\left(t\left(k_{\star}\right)\right)=1.98 \times 10^{-7} .
\end{array}
$$

where the 'dot' refers to the cosmic time. Because of observational error bars, these values are uncertain in $\mathrm{a} \approx 2 \%$ window. We will use these values in our analysis and ask at the end whether the quantum state of perturbations at the onset of the slow roll is indistinguishable from the $\mathrm{BD}$ vacuum. In most of the parameter space that dictates the initial conditions at the bounce, the answer will be in the affirmative. For a narrow window in which it is in the negative, one would have to re-calculate values of these parameters (see Section V A).

Our task then is to find the class of initial conditions for the effective equations for which dynamical trajectories enter the tiny region of the phase space defined by (3.2) and the associated error bars, some time in their future evolution. As in general relativity, the space of initial data in the effective theory is 4 dimensional: specification of $\nu, \mathrm{b} ; \phi, p_{(\phi)}$ at any time determines a unique solution to effective equations. (Recall that $\nu \sim a^{3}$ and $H \sim \sin 2 \lambda \mathrm{b}$.) At the bounce, we have $\lambda \mathrm{b}=\pi / 2$ and furthermore, as in general relativity, we have two symmetries on the space of solutions: i) a rescaling symmetry, $\nu(t) \rightarrow \alpha \nu(t), \phi(t) \rightarrow \phi(t)$, and, ii) and the time reflection symmetry $\nu(t) \rightarrow \nu(-t), \phi(t) \rightarrow \phi(-t)$. The first allows us to restrict ourselves to solutions with $\left.\nu\right|_{\mathrm{B}}=\nu^{o}$ for some fixed constant $\nu^{o}$, while the second lets us focus just on solutions with $\left.\dot{\phi}\right|_{\mathrm{B}}>0$; solutions with arbitrary $\left.\nu\right|_{\mathrm{B}}$ and also those with $\left.\dot{\phi}\right|_{\mathrm{B}}<0$ can be obtained using these symmetries. Finally, the LQC Friedmann equation (2.10) implies that the value $\phi_{\mathrm{B}}$ of $\phi$ at the bounce point determines $|\dot{\phi}|_{\mathrm{B}}$ there. Thus, the free data at the bounce is just the value $\phi_{\mathrm{B}}$ of the background inflaton. Next, since the total

\footnotetext{
${ }^{8} k_{\star}$ is given by $k_{\star} / a_{o}=2 \times 10^{-3} \mathrm{Mpc}^{-1}$, or, $k_{\star}=8.58 k_{o}$, where $a_{o}$ refers to the scale factor today and, as before, $k_{o}$ refers to the wave number that has just re-entered the Hubble radius today. It is only the combination $k_{\star} / a_{o}$ that has direct physical meaning; $2 \pi a_{o} / k_{\star}$ is the physical wave length of this reference mode today.
} 
energy density at the bounce is fixed, $\rho=\rho_{\max }$ on all solutions, it follows that this free data $\phi_{B}$ is restricted to lie in a finite interval:

$$
\left|\phi_{\mathrm{B}}\right| \in\left(0, \phi_{\max }\right), \quad \text { where } \quad \phi_{\max }=\frac{0.90}{m} \approx 7.47 \times 10^{5} .
$$

This interval provides us with the relevant parameter space in this paper.

For definiteness, we will assume $\phi_{\mathrm{B}}>0$ since the sign does not make a qualitative difference to the analysis. In this case, it was shown in [6] that a sufficient condition for the trajectory to enter the tiny phase space region compatible with the 'WMAP slow roll' is that $\phi_{B} \geq 0.93$. This excludes only a tiny part of the full range of allowed values. In this sense, in the effective theory, 'almost all' initial conditions at the bounce point are compatible with the 7 year WMAP data. We would like to emphasize that this behavior is very nontrivial. At first sight it may seem that the statement just says that, as in general relativity, inflationary trajectories are attractors in effective LQC. However, the implication is much stronger because compatibility with the seven year WMAP data is a sharp quantitative requirement: We need the trajectory to achieve $\phi=3.15$ and $\epsilon=8 \times 10^{-3}$ at the time when $H=7.83 \times 10^{-6}$ within the WMAP error bars. Second, as we saw in section II B 2, preinflationary dynamics of effective LQC have several features that are very distinct from what occurs in general relativity. Therefore, a priori one cannot assume that even the attractor property of general relativity must carry over. Finally the pre-inflationary dynamics covers some 11 orders of magnitude in matter density and curvature. Therefore it is rather striking that 'almost all' effective trajectories starting at the bounce would enter the tiny region of phase space compatible with the onset of the desired slow roll.

From now on we will restrict ourselves to this part, $\phi_{\mathrm{B}} \geq 0.93$, of the parameter space. The restriction involved is tiny. However, this does not imply that the initial conditions for the quantum state $\Psi_{o}$ at the bounce are generic. The permissible quantum states are very special because we have asked that $\Psi_{o}$ be sharply peaked at a point on the constraint surface of the phase space of the effective theory at the bounce time. What is generic is only the point at which they are peaked.

We will conclude by clarifying a subtlety about what it means to have the initial state $\Psi_{o}\left(\nu, \phi_{\mathrm{B}}\right)$ at the bounce to be peaked at a point $\left(\nu^{o}, \mathrm{~b}=\pi / 2 \lambda ; \phi_{\mathrm{B}}^{o}, p_{(\phi)}^{o}\right)$ on the constraint surface. Note first that for any given $\phi_{\mathrm{B}}^{o}$ and $\nu^{o}$, the Hamiltonian constraint (2.10) in the effective theory determines $p_{(\phi)}^{o}$. The subtlety is that, as noted in section IIB, the Hamiltonian constraint is de-parameterized on the physical sector: Since $\phi$ serves as 'internal time', it is a parameter rather than an operator on the physical Hilbert space $\mathcal{H}_{o}$. However, because of the quantum Hamiltonian constraint, $\hat{p}_{(\phi)} \Psi_{o}=-\hat{H}_{o} \Psi_{o}, \hat{p}_{(\phi)}$ is a well-defined operator on $\mathcal{H}_{o}$. Therefore, the initial state $\Psi_{o}\left(\nu, \phi_{\mathrm{B}}\right)$ is to be chosen so that the expectation

value of $\hat{\nu}$ is $\nu^{o}$, that of $\hat{H}_{o}$ is the $p_{(\phi)}^{o}$ determined by the chosen $\phi_{\mathrm{B}}^{o}$, and fluctuations in both quantities are very small. For further details on construction of such states, see [22].

\section{B. Initial conditions for the quantum state $\psi$ of perturbations}

For linear test fields in de Sitter space-time, one can single out a unique state by demanding that it be regular and invariant under the full de Sitter group. This is the BD vacuum. Since in the slow roll inflationary phase the Hubble parameter is approximately constant, space-time geometry is well approximated by the de Sitter metric. This motivates the usual 
choice of the BD vacuum as the quantum state of perturbations at the onset of inflation. However, in the pre-inflationary phase, especially near the bounce, space-time geometry is very far from the de Sitter geometry. As we noted in section II B 2, the Hubble parameter vanishes at the bounce. It then increases very quickly to attain a Planck scale value (in less than a Planck second for kinematic energy dominated bounces!) [6]. Therefore, it is not possible to even formulate the notion of an approximate BD vacuum at or near the bounce.

However, we can still carry over the central idea that led to the choice of the BD vacuum in the standard inflationary scenario and ask that the initial state $\psi\left(\mathcal{T}_{\vec{k}}, \mathcal{Q}_{\vec{k}}\right)$ of perturbations be regular and maximally symmetric. Since the state $\Psi_{o}$ and hence the dressed effective metric $\tilde{g}_{a b}$ is invariant under the FLRW isometries, the symmetry requirement is precisely that $\psi\left(\mathcal{T}_{\vec{k}}, \mathcal{Q}_{\vec{k}}\right)$ be invariant under spatial translations and rotations. We are thus led to ask that $\psi\left(\mathcal{T}_{\vec{k}}, \mathcal{Q}_{\vec{k}}\right)$ be a 4 th order adiabatic vacuum at the bounce time $\tilde{\eta}=\tilde{\eta}_{\mathrm{B}}$. In the Schrödinger picture, the state will evolve but retain its 4 th order regularity and invariance under rotations and translations (see Eq (2.21)). In the Heisenberg picture, of course, it is manifest that the conditions are not tied to any specific time.

Although we have imposed maximal possible symmetry requirements that are compatible with pre-inflationary physics, as our discussion of section II C shows, the space of permissible states is still infinite dimensional. A second requirement on the choice of the initial $\psi$ is motivated by our truncation approximation. Clearly, for the approximation to be meaningful, the initial perturbed state $\psi$ should be such that its contribution $\left\langle\psi\left|\hat{\rho}\left(\vec{x}, \tilde{\eta}_{\mathrm{B}}\right)\right| \psi\right\rangle_{\text {ren }}$ to the energy density at the bounce time $\tilde{\eta}_{\mathrm{B}}$ should be negligible compared to $\rho_{\max }$, the energy in the background quantum geometry $\Psi_{o}$. In contrast to the regularity and symmetry, this last requirement is tied to a specific time, $\tilde{\eta}=\tilde{\eta}_{\mathrm{B}}$.

A key question is whether states satisfying both these conditions exist. Detailed calculations using 4th order adiabatic regularity show that they do. A particularly convenient choice for numerical simulations can be arrived at as follows. For definiteness, let us consider tensor modes. Then, in the large $k$ limit, any 4 th adiabatic order basis $e_{k}(\tilde{\eta})$ has to approach the explicitly known approximate (WKB) solutions $f_{k}^{(4)}(\tilde{\eta})$ to $(2.18)$ faster than $(\tilde{a} / k)^{9 / 2}$. The $f_{k}^{(4)}(\tilde{\eta})$ are given by $[19]$

$$
f_{k}^{(4)}(\tilde{\eta})=\frac{1}{\tilde{a}(\tilde{\eta}) \sqrt{2 W_{k}^{(4)}(\tilde{\eta})}} e^{-i \int^{\tilde{\eta}} W_{k}^{(4)}(\tau) d \tau}
$$

where $W_{k}^{(4)}(\tilde{\eta})=\sum_{i=1}^{4} W_{i}$, with

$$
\begin{aligned}
& W_{0}=k ; \quad W_{1}=0 ; \quad W_{2}=-\frac{1}{2 k} \frac{\tilde{a}^{\prime \prime}}{\tilde{a}} \\
& W_{3}=0 ; \quad W_{4}=\frac{2 \tilde{a}^{\prime \prime} \tilde{a}^{\prime 2}-2 \tilde{a}^{\prime \prime 2} \tilde{a}-2 \tilde{a} \tilde{a}^{\prime} \tilde{a}^{\prime \prime \prime}+\tilde{a}^{2} \tilde{a}^{\prime \prime \prime \prime}}{8 k^{3} \tilde{a}^{3}}
\end{aligned}
$$

The leading order term of (3.4) corresponds to the positive frequency solution in Minkowski space and the rest of the terms are higher order contributions that vanish at different rates when $(\tilde{a} / k) \rightarrow 0$. Therefore, a natural strategy is to construct an 'obvious' 4th order regular basis $e_{k}^{\text {obv }}(\tilde{\eta})$, tailored to the bounce time $\tilde{\eta}_{\mathrm{B}}$, as follows. Although the definition of 4 th order adiabaticity is only an asymptotic one, we can ask that $e_{k}^{\text {obv }}(\tilde{\eta})$ and $f_{k}^{(4)}(\tilde{\eta})$ share the same initial data at the bounce time $\tilde{\eta}=\tilde{\eta}_{\mathrm{B}}$ :

$$
e_{k}^{\text {obv }}\left(\tilde{\eta}_{\mathrm{B}}\right)=f_{k}^{(4)}\left(\tilde{\eta}_{\mathrm{B}}\right) ; \quad \text { and } \quad \partial_{\tilde{\eta}} e_{k}^{\mathrm{obv}}\left(\tilde{\eta}_{\mathrm{B}}\right)=\partial_{\tilde{\eta}} f_{k}^{(4)}\left(\tilde{\eta}_{\mathrm{B}}\right) .
$$


(This relation will not hold at future times because $e_{k}^{\text {obv }}$ satisfies (2.18) exactly while $f_{k}^{(4)}$ satisfies it only approximately.) The vacuum state $\left|0^{\text {obv }}\right\rangle$ determined by $e_{k}^{\text {obv }}$ will be referred to as the 'obvious 4th adiabatic order vacuum'.

There is one subtlety: For this construction to work, $W_{k}^{(4)}\left(\tilde{\eta}_{\mathrm{B}}\right)$ in $(3.4)$ must be nonnegative since it appears under a square-root in the expression of $f_{k}^{(4)}\left(\tilde{\eta}_{\mathrm{B}}\right)$. This is the case when the infra-red cut-off is not too low. In this case, detailed numerical calculations have shown that $\left\langle 0^{\text {obv }}\left|\hat{\rho}\left(\vec{x}, \tilde{\eta}_{\mathrm{B}}\right)\right| 0^{\text {obv }}\right\rangle_{\text {ren }}$ is less than a few percent of the background energy density $\rho_{\max }$. If the infrared cut-off is lower, on the other hand, one has to modify the procedure to obtain the initial data for $e_{k}^{\text {obv }}$ : It is to be given by a suitable smooth extension of $f_{k}^{(4)}$ and its time derivative from the high $k$ values where $W_{k}^{(4)}\left(\tilde{\eta}_{\mathrm{B}}\right)$ is non-negative to the lower $k$ values where it becomes negative. Consequently, $\left|0^{\text {obv }}\right\rangle$ is not as 'canonical'. However, the required extension can again be carried out keeping the renormalized energy density in perturbations only a few percent of that in the background.

The non-trivial question is whether the energy density in the perturbation continues to remain negligible for $\tilde{\eta}>\tilde{\eta}_{\mathrm{B}}$. The issue is especially non-trivial in the Planck regime. However, using numerical evolution we will show in section $\mathrm{V}$ that the answer is in the affirmative for the state $\left|0^{\text {obv }}\right\rangle$ at least when $\phi_{\mathrm{B}}>1.23$. Furthermore, we will argue analytically that the same is true for states in an open (infinite dimensional) neighborhood of $\left|0^{\text {obv }}\right\rangle$. Thus, states satisfying all our initial conditions at the bounce do exist.

Remark: Physically, the IR cut-off is dictated by the physical radius $\mathfrak{R}_{\mathrm{LS}}$ of the observable universe at the surface of last scattering (see section II C2). More precisely it is the comoving radius $\mathfrak{R}_{\mathrm{IR}}$ such that $\mathfrak{R}_{\mathrm{LS}}=\left(\tilde{a}\left(\tilde{\eta}_{\mathrm{LS}}\right)\right) \mathfrak{R}_{\mathrm{IR}}$. Since $\mathfrak{R}_{\mathrm{LS}}$ is observationally fixed, the value of $\mathfrak{R}_{\mathrm{IR}}$ is sensitive to the number of e-foldings between the bounce and the surface of last scattering. Now, since the background states $\Psi_{o}$ under consideration all meet conditions that are compatible with the 7 year WMAP data at the onset of slow roll, the number of e-foldings between this onset and the surface of last scattering is the same for them. What differs from one $\Psi_{o}$ to another is the number of e-foldings between the bounce and the onset of slow roll; they grow rapidly as $\phi_{\mathrm{B}}$ increases. Therefore the subtlety in the choice of mode functions mentioned above arises only for low values, $\phi_{\mathrm{B}} \leq 1.18$, for which the number of e-foldings between the bounce and the onset of inflation is low.

\section{Physical consideration}

In this paper, our primary goal is to explore the extent to which the inflationary scenario can be extended to the Planck regime. We saw that, in effective LQC, a very large portion $\mathfrak{P}$ of the allowed initial data at the bounce leads to dynamical trajectories that encounter a slow roll phase compatible with the 7 year WMAP data. This led us to choose an initial state $\Psi_{o}$ of the background geometry to be sharply peaked at a point of $\mathfrak{P}$. The initial conditions on the states $\psi$ of perturbations were motivated by three considerations: i) Regularity; ii) Symmetry; and iii) Compatibility with our truncation approximation. The first two conditions led us to restrict $\psi$ to be a 4th adiabatic order 'vacuum' state and the third led us require that the energy density in $\psi$ be negligible compared to that in background quantum geometry $\Psi_{o}$ at the bounce. This set of conditions is not overly restrictive in the sense that they allow an infinite set of initial states. At the same time, because $\Psi_{o}$ is required to be sharply peaked at a point in $\mathfrak{P}$, and because $\psi$ has to satisfy the three conditions at 
the bounce, the allowed initial states $\Psi_{o} \otimes \psi$ constitute a very tiny subset of all states in $\mathcal{H}_{o} \otimes \mathcal{H}_{1}$. In this sense they are very special. We will now provide heuristic considerations to help clarify the physical motivation that underlies our choices. This discussion could also serve to further narrow down the initial conditions and opens some avenues for further work.

A common strategy, articulated by Penrose [73] in particular, is to use known physics and our knowledge of the current universe to draw conclusions about the special nature of initial conditions. In LQC this general idea has been used to narrow down the choice of the initial quantum state $\Psi_{o}$ of the background geometry [7]. As noted in section III A, one begins with the observation that, away from the deep Planck regime, the universe is extremely well-described by a FLRW solution of Einstein's equation. One therefore takes states in $\mathcal{H}_{o}$ which are sharply peaked on a FLRW solution and evolves them back in time. One finds that the states continue to be sharply peaked with small uncertainties in both 'conjugate' variables $\hat{H}_{o}$ and $\hat{\nu}$, all the way to the bounce. It is this property that motivates our initial conditions on $\Psi_{o}$ at the bounce.

The choice of $\psi$ can be further narrowed down by similar considerations. In any of our vacua $|0\rangle$ for perturbations, the expectation values of $\hat{\mathcal{T}}_{\vec{k}}, \hat{\mathcal{Q}}_{\vec{k}}$ and their conjugate momenta vanish, encoding the idea that departures from homogeneity and isotropy are small. But we could also ask that the states be 'as peaked on the zero values as is allowed by the uncertainty principle'. There is a precise sense in which the state $\left|0^{\text {obv }}\right\rangle$ we discussed in some detail in section III B satisfies this condition. Heuristically, this condition would say that the initial state $\Psi_{o} \otimes \psi$ is as homogeneous and isotropic as is permissible in the quantum physics of the truncated theory. Therefore this condition will be referred to as quantum homogeneity and isotropy at the bounce. A precise formulation and implications of this requirement will be discussed in detail in [74].

As we explained in the beginning of this section, our goal is only to show the existence of initial conditions that lead to a self-consistent completion of the standard inflationary paradigm and we will see in the next two sections that quantum homogeneity and isotropy at the bounce meets this goal. In future and more comprehensive investigations one could inquire if one can arrive at this condition from deeper considerations. Specifically, since the background quantum geometry $\Psi_{o}$ has a pre big bang branch, one can ask if the initial conditions at the bounce naturally arise from the prior evolution. We will conclude by providing some heuristics that suggest a promising direction to address this issue.

First, if $\phi_{\mathrm{B}} \geq 1.15$ (the value used in most numerical plots in this paper), the universe that is observable at the surface of last scattering expanded from a ball of radius $r_{\mathrm{B}}<10 \ell_{\mathrm{Pl}}$ at the bounce. Therefore, to account for the CMB observations, one needs quantum homogeneity and isotropy at the bounce only at this small scale. At first, this smallness of scale may appear to make the requirement quite weak. Indeed, such an argument is sometimes made in the standard inflationary scenario where one assumes the validity of general relativity all the way to the Planck scale and argues that a similar small region expands out to fill the universe that is observable at the surface of last scattering. However, it has been pointed out by many authors, on the initial ball the required homogeneity and isotropy has to be extraordinarily high and a natural mechanism to achieve this does not appear in the standard scenario. We will now give qualitative arguments to the effect that the underlying quantum geometry of LQG may provide the missing element.

Prior to the bounce, the universe would be collapsing thereby producing strong inhomogeneities that lead to complicated growth of curvature. However, the LQC models that have been analyzed in detail suggest the following overall picture. Quantum geometry effects 
lead to a new repulsive force. This force is completely negligible until a curvature scalar approaches the Planck regime. But then it grows very quickly, overwhelms the classical gravitational attraction that would have made the curvature scalar singular, and dilutes its value. Thus, in addition to the 'global' density bounce we have focused on, there would be many 'local' bounces associated with local growth of curvature. The quantum geometry corrections start to be significant when the curvature is $\sim 10^{-2} \ell_{\mathrm{Pl}}^{-2}$, (i.e. about a thousandth of the maximum value) which corresponds to a radius of curvature of about $10 \ell_{\mathrm{Pl}}$. Therefore, one may expect the 'dilution' mechanism to be effective in ironing out the 'wrinkles' in the curvature also on that scale. Thus, the missing element in achieving the required quantum homogeneity and isotropy on the $10 \ell_{\mathrm{Pl}}$ scale could well be provided by the repulsive forces that originate in quantum geometry. (See [64, 65] for a semi-classical mechanism leading to desired initial conditions in a different context.) This mechanism would not preclude inhomogeneities and anisotropies on larger scales at the bounce. But these would correspond to modes whose wavelength at the surface of last scattering is much greater than the radius of the observable universe and would not be observable. If these considerations are borne out, the pre-bounce history of the background would not have a direct relevance to the post-bounce evolution of the portion of the universe that is observable.

Of course a much more detailed investigation of this 'dilution effect' is needed to determine if these qualitative ideas are viable and can be developed into a detailed quantitative argument.

Remark: If we just focus on the tensor perturbations, then the requirement of quantum homogeneity and isotropy at the bounce implies that the state $\Psi_{o}(\nu) \otimes \psi\left(\mathcal{T}_{\vec{k}}\right)$ satisfies a quantum version of Penrose's Weyl curvature hypothesis [73] in the following sense. In its original formulation, space-time geometry was assumed to be classical, and the condition was that the initial singularity was very special in that the Weyl curvature vanishes there. In LQC of course there is no singularity and hence it is natural to impose a condition at the bounce surface at which the matter density achieves its maximum value. Furthermore, since the electric and the magnetic parts of the Weyl tensor, $\hat{E}_{a b}, \hat{B}_{a b}$, do not commute in the truncated theory, and their commutator is a c-number, there is no state on which they can both vanish. Thus, the Heisenberg uncertainty principle tells us that it is not meaningful to ask that the Weyl tensor operator be zero even at an instant of time. This is completely analogous to what happens in the Maxwell theory in Minkowski space, where there is no state at all in the Fock space that is annihilated by the Maxwell field operator $\hat{F}_{a b}$, or equivalently, the electric and magnetic field operators $\hat{E}^{a}, \hat{B}^{a}$. The expectation values of both these operators can vanish but this can be achieved on a very large class of states. But we can impose, in addition, the next natural requirement: i) the product of uncertainties in the electric and magnetic fields be saturated; and, ii) the uncertainties be divided equally between the two fields. Some care is required in formulating these conditions in a precise manner. When this is done, one finds that there is exactly one state that satisfies the condition: The standard Maxwell vacuum! In the truncated theory now under considerations, it is natural to elevate Penrose's Weyl curvature hypothesis to the quantum theory by asking for states $\psi$ in which the expectation values of $\hat{E}_{a b}, \hat{B}_{a b}$ vanish, the product of uncertainties is minimized and the uncertainties are equally divided between the electric and magnetic parts. This quantum version of the Weyl curvature hypothesis is satisfied in a weaker sense (i.e., only in the adiabatic limit) by states $\Psi_{o} \otimes \psi$ which meet our quantum homogeneity and isotropy requirement. (For details, see [74].) 


\section{PRE-INFLATIONARY DYNAMICS OF THE QUANTUM CORRECTED BACKGROUND}

This section is divided into two parts. In the first we discuss the interplay between the dynamics of the background curvature and that of modes of perturbations in physical terms. In the second we describe results of numerical simulations of the evolution of the effective background geometry.

To discuss dynamics in detail, one has to choose a time parameter defined by the effective background metric. In the cosmology literature one generally uses conformal time $\eta$ which moves the big bang to $\eta=-\infty$. In cosmic time $t$, it occurs at $t=0$. Now, we would like to start our evolution at the LQC big bounce which replaces the big bang of general relativity. To compare the results with those of general relativity, it is technically more convenient to use the cosmic time $\tilde{t}$ defined by the $\tilde{g}_{a b}$ and set the bounce time to be $\tilde{t}_{\mathrm{B}}=0$. Therefore, while our analytical considerations use conformal time $\tilde{\eta}$, our numerical evolutions are all carried out in cosmic time $\tilde{t}$.

\section{A. Why pre-inflationary dynamics matter}
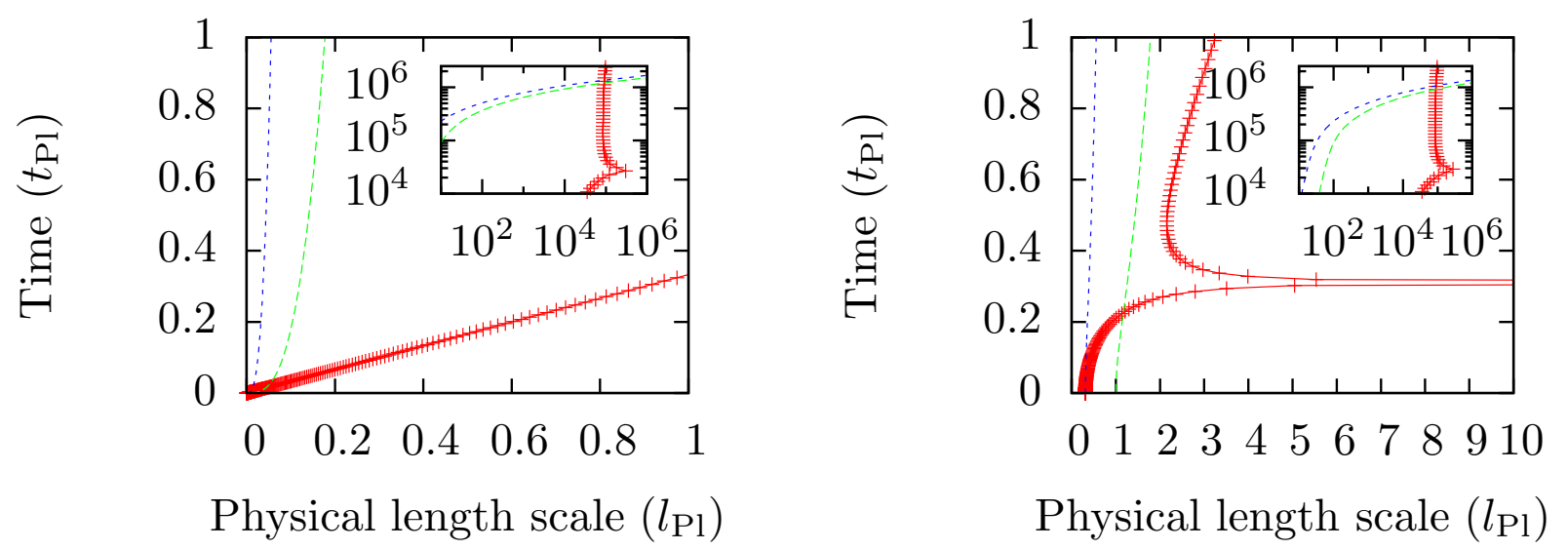

FIG. 1: Time evolution of the curvature radius (red solid line with dashes) and of a wave length of interest to observations (green dashed line). Left Panel: General relativity. The modes of interest have wave lengths less than the curvature radius all the way from the big bang $(\tilde{t}=0)$ until after the onset of slow roll, shown in the inset $\left(\tilde{t} \sim 10^{5} t_{\mathrm{Pl}}\right)$. Right panel: LQC. The bounce occurs at time $t=0$ and we have set $\left.\tilde{a}\right|_{\tilde{t}=0}=1$. The blue dotted line (extreme left) shows the evolution of the mode whose wave length $\left.\lambda_{\text {phy }}\right|_{\tilde{t}=0}$ at the big bounce equals the curvature radius. This mode and modes with smaller wavelengths remain within the curvature radius until the onset of inflation. On the other hand, modes with physical wave length greater than the curvature radius at the bounce (for example green dashed line) enters the curvature radius soon after the bounce and remain within the curvature radius until after the onset of inflation, shown in the inset $\left(\tilde{t} \sim 10^{5} t_{\mathrm{Pl}}\right)$. These modes can be excited due to curvature while their wave length is greater than the curvature radius and will not be in the $\mathrm{BD}$ vacuum at the onset of inflation. (There are two points $\left(\right.$ at $\tilde{t} \approx 0.3 t_{\mathrm{Pl}}$ and $\left.\tilde{t} \approx 5 \times 10^{4} t_{\mathrm{Pl}}\right)$ in the LQC evolution at which the $w=1 / 3$ in the effective equation of state, whence the scalar curvature vanishes and the radius of curvature goes to infinity.) 
There is a common lore that inflation would simply dilute away all the effects of preinflationary dynamics. This belief stems from the following physical considerations. For definiteness let us consider tensor perturbations. To bring out the physics that governs evolution, it is convenient to simplify the dynamical equation by noting the following mathematical fact. Under a general conformal transformation $\stackrel{\circ}{g}_{a b}=\Omega^{-2} \tilde{g}_{a b}$ we obtain $(\widetilde{\square}-(1 / 6) \tilde{R}) \tilde{\phi}=\Omega^{-3}(\stackrel{\circ}{\square}-(1 / 6) \stackrel{\circ}{R}) \phi$ where $\tilde{\phi}=\Omega^{-1} \phi$. Now, if we let $\Omega=\tilde{a}$, then $\stackrel{\circ}{g}_{a b}$ is the flat metric defined by the coordinates $\tilde{\eta}, \vec{x}$ and we have $\widetilde{\square} e_{k}=\tilde{a}^{-3}\left[\square^{\circ}+(1 / 6) a^{2} \tilde{R}\right]\left(a e_{k}\right)=0$. Therefore, the fact that $e_{k}$ satisfies the wave equation $\widetilde{\square} e_{k}=0$ w.r.t. $\tilde{g}_{a b}$ (see (2.18)) implies that the rescaled function $\chi_{k}(\tilde{\eta})=\tilde{a}(\tilde{\eta}) e_{k}(\tilde{\eta})$, satisfies a wave equation w.r.t. the flat metric $\stackrel{\circ}{g}_{a b}$ in presence of a time dependent potential $(1 / 6) \tilde{a}^{2} \tilde{R}$ :

$$
\partial_{\tilde{\eta}}^{2} \chi_{k}(\tilde{\eta})+\tilde{a}^{2}(\tilde{\eta})\left(\frac{k^{2}}{\tilde{a}(\tilde{\eta})^{2}}-\frac{\tilde{R}(\tilde{\eta})}{6}\right) \chi_{k}(\tilde{\eta})=0,
$$

where $\tilde{R}(\tilde{\eta})$ is the scalar curvature. Eq. (4.1) brings out the fact that for modes with physical wave numbers $k / \tilde{a}$ much larger than the curvature energy-scale $k_{R}=(\tilde{R} / 6)^{1 / 2}$, the effect of curvature on their evolution can be ignored. Put differently, if the physical wave length $\lambda_{\text {phy }}=2 \pi \tilde{a} / k$ of the mode is much smaller than the radius of curvature $(6 / \tilde{R})^{1 / 2}$, the mode propagates as if it is in flat space-time (defined by $\tilde{\eta}_{a b}$ ); its dynamics is trivial. In the standard inflationary scenario, modes that can be observed in the CMB have physical wave length smaller than the curvature radius at the onset of inflation. ${ }^{9}$ If one were to use general relativity to evolve back in time, then this inequality can continue to hold all the way to the big bang (see the left panel in Fig. 1). Therefore, the modes would remain in the same quantum state throughout the pre-inflationary era, whence one would conclude that the pre-inflationary dynamics does not have any effect on modes that are observable in the CMB.

What is the situation in LQC? Because of the exact equivalence between the evolution of perturbations $\hat{\mathcal{T}}$ on the quantum geometry $\Psi_{o}$ and on the dressed-effective metric $\tilde{g}_{a b}$ given by Eq. (2.12), these general considerations continue to hold all the way back to the bounce. However, the pre-inflationary dynamics of the scale factor $\tilde{a}(\tilde{\eta})$ and the curvature $\tilde{R}(\tilde{\eta})$ is now qualitatively different. The key question then is: Do the physical wavelengths $\lambda_{\text {phy }}$ of the relevant modes ever become comparable to $(6 / \tilde{R})^{1 / 2}$ during the pre-inflationary evolution? If they do, their dynamics would be non-trivial.

A detailed analysis shows that this is the case for modes whose physical wave length $\lambda_{\text {phy }}$ exceeds the curvature radius at the bounce time (see the right panel of Fig. 1). As Parker $[68,70]$ showed already in the sixties, modes that experience curvature are excited. Indeed, during slow roll, this is the phenomenon that leads to the inhomogeneities that seed the large scale structure. Qualitatively the situation is similar in the pre-inflationary epoch as well: modes with $\left.\lambda_{\text {phy }}\right|_{t=0}>\left.(6 / \tilde{R})^{1 / 2}\right|_{t=0}$ are excited in the Planck regime that immediately follows the bounce. As a consequence, at the onset of inflation, the quantum state of perturbations is populated by excitations of these modes over the BD vacuum. Thus, pre-inflationary dynamics can change the initial conditions for perturbations at the

\footnotetext{
${ }^{9}$ For (quasi-) de Sitter space-times this is equivalent to requiring $\lambda \ll \mathfrak{R}_{H}$ where $\mathfrak{R}_{H}=1 / H$ is the Hubble radius. This is the condition generally discussed in the inflationary literature. However, away from slow roll, $\mathfrak{R}_{H}$ is not so simply related to the radius defined by the curvature scale.
} 
onset of the slow roll. Can this lead to physically distinct consequences? The answer is in the affirmative. It has been shown that the predictions for the CMB and for the distribution of galaxies are sensitive to the quantum state of perturbations at the beginning of inflation [10-14]. Furthermore, concrete observational tests to probe these consequences have recently been proposed [15-17].

To summarize, pre-inflationary dynamics can lead to predictions that are distinct from that of the standard inflation. In principle, there may be excitations over the BD vacuum at the onset of inflation which are so large as to be in conflict with the observed power spectrum and spectral index of the scalar perturbations. If this occurs, the corresponding initial conditions at the bounce would be ruled out observationally. If the predictions are compatible with current observations, the departure from the BD vacuum can still lead to effects that could be observed in the future. This possibility is of special interest because it relates the Planck scale dynamics to observations. A priori we do not know if any or all of these possibilities would be realized for the permissible values of the free parameter of the theory, $\phi_{B}$. These questions can be answered only by a detailed analysis of the pre-inflationary dynamics.

\section{B. Quantum corrected background geometry}

Recall from section II C 1 that, because $\Psi_{o}$ is so sharply peaked, within numerical errors, one can replace the dressed effective metric $\tilde{g}_{a b}$ with the $\bar{g}_{a b}$ that traces the evolution of the peak of $\Psi_{o}$ and satisfies effective equations. This is a set of three equations. The first is the Hamiltonian constraint (2.11) that must be satisfied at any instant of time and the other two provide evolution:

$$
\begin{aligned}
& \ddot{\nu}=\frac{24 \pi \nu}{\rho_{\max }}\left[(\rho-V(\phi))^{2}+V(\phi)\left(\rho_{\max }-V(\phi)\right)\right] \\
& \ddot{\phi}+\frac{\dot{\nu}}{\nu} \dot{\phi}+V_{, \phi}=0,
\end{aligned}
$$

where as usual the 'dot' denotes derivative with respect to comic time $\tilde{t}$. As discussed in section III A, at the bounce time the only free parameter is the value $\phi_{\mathrm{B}}$ of the background inflaton which is constrained to lie in the interval $\phi_{\mathrm{B}} \in\left(0.93,7.41 \times 10^{5}\right)$. For each choice of $\phi_{\mathrm{B}}$ in this interval, we obtain a solution to the effective equations which enters the slow roll compatible with the 7 year WMAP data some time in its future evolution.

The dynamical equations (4.2) constitute a set of coupled ordinary differential equations. They were solved using Mathematica. As an accuracy check, the satisfaction of the Hamiltonian constraint (2.11) was monitored throughout the evolution. For reasons mentioned in section III A and will become clearer in this subsection, in this discussion we will pay special attention to the case when the bounce is kinetic energy dominated.

The bounce occurs at $\tilde{t}=0$ and we use the convention $\left.\tilde{a}\right|_{\tilde{t}=0}=1$ (rather than $\tilde{a}_{\text {today }}=1$ ). Immediately after the bounce there is a phase of super-inflation -i.e. faster than exponential expansion - because $\dot{H}>0$ at the bounce and $H>0$ immediately after the bounce in all solutions. At the end of this super-inflation phase, the Hubble parameter achieves its maximum value $H_{\max } \approx 0.93$. For bounces in which the kinetic energy dominates, the superinflation phase is dominated by the Planck scale dynamics because the Hubble parameter grows from zero to its maximum value in a fraction of a Planck second. This extremely 


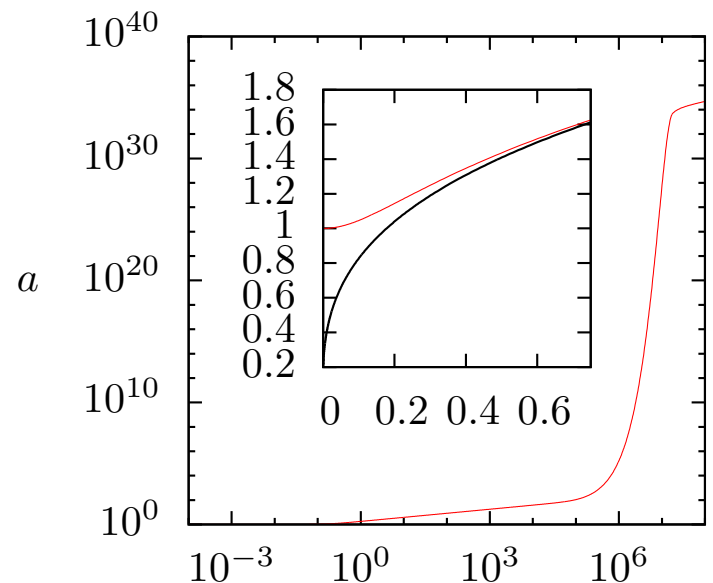

Cosmic time (Planck seconds)

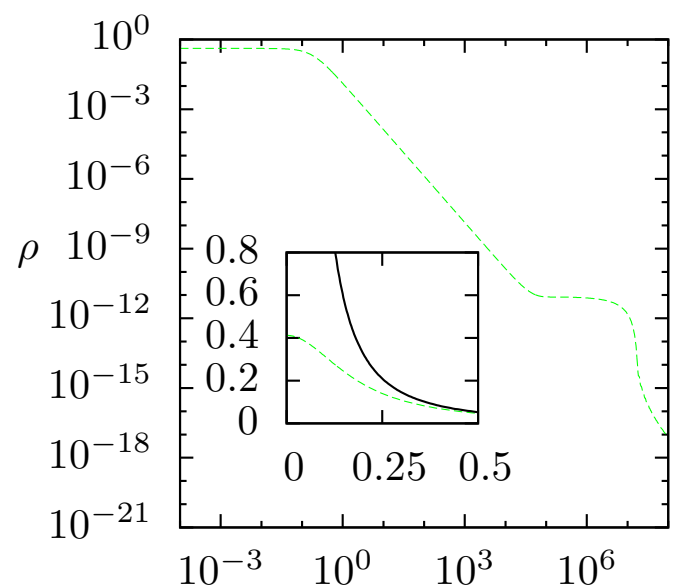

Cosmic time (Planck seconds)

FIG. 2: Evolution of the scale factor $\tilde{a}$ (left) and the energy density $\rho$ (right) as a function of cosmic time for $\phi_{\mathrm{B}}=1.15$. Slow roll occurs around $\tilde{t} \sim 10^{6} t_{\mathrm{Pl}}$ and during this phase the scale factor increases exponentially and the density remains nearly constant. The insets in both plots highlight the behavior near $\tilde{t}=0$. The lower curve in the left inset and the upper in the right inset show the behavior in general relativity in which the scale factor goes to zero and the energy density diverges. By contrast in LQC (upper curve in the left inset and the lower curve in the right inset) both quantities remain finite at the bounce.

short lived phase is followed by a much longer phase during which the LQC corrections to dynamics become progressively weaker as the inflaton loses kinetic energy because the friction term (due to the Hubble parameter) is large; it lasts till $\tilde{t} \sim 10^{4} t_{\mathrm{Pl}}$. At the end of this phase, the kinetic energy in the inflaton is equal to the potential energy and the total energy density is about $10^{-10} \rho_{\mathrm{Pl}}$ (and hence the curvature has also fallen by ten orders of magnitude). The inflaton is still going up the potential at the end of this phase. It continues to lose kinetic energy and at $\tilde{t} \sim 10^{5} t_{\mathrm{Pl}}$, it stops climbing, turns around and starts descending the potential. Soon there after, when $\tilde{t} \sim 10^{5}-10^{6} t_{\mathrm{Pl}}$, it enters the slow roll inflation compatible with the 7 year WMAP data. The slow roll phase lasts for about the same time interval as it takes to reach that phase starting from the bounce through pre-inflationary dynamics. Fig. 2 illustrates the dynamical evolution of the scale factor and energy density using $\phi_{\mathrm{B}}=1.15$. (See also [6].)

To make contact with the WMAP phenomenology, we need to locate the reference mode $k_{\star}$. We fix a value of $\phi_{\mathrm{B}}$ and obtain the corresponding solution $\tilde{a}(\tilde{t}), \phi(\tilde{t})$. We then monitor values of the Hubble parameter $H(\tilde{t})$ and locate the time $\tilde{t}\left(k_{\star}\right)$ at which it assumes the reference value $H\left(\tilde{t}\left(k_{\star}\right)\right)=7.83 \times 10^{-6}$ determined by the WMAP data (see $\left.(3.2)\right)$. Then $k_{\star}$ is the co-moving wave number of the mode whose physical wave number equals the Hubble parameter at this time: $k_{\star} / \tilde{a}\left(\tilde{t}\left(k_{\star}\right)\right)=7.83 \times 10^{-6}$. This procedure determines both $k_{\star}$ and the time $\tilde{t}\left(k_{\star}\right)$ at which the reference mode $k_{\star}$ exits the Hubble radius for any given value of $\phi_{B}$. As Fig. 3 shows, $k_{\star}$ increases very rapidly with $\phi_{\mathrm{B}}$. We also calculate $\epsilon\left(\tilde{t}\left(k_{\star}\right)\right)$ and verify that it is in a small window around $8 \times 10^{-3}$ in order to ensure that the slow roll phase is compatible with the 7 year WMAP data within its error bars. As a check on numerics, we also verify that $\phi\left(\tilde{t}\left(k_{\star}\right)\right)$ and $\dot{\phi}\left(\tilde{t}\left(k_{\star}\right)\right)$ are in agreement with (3.2). Finally, we calculate the 


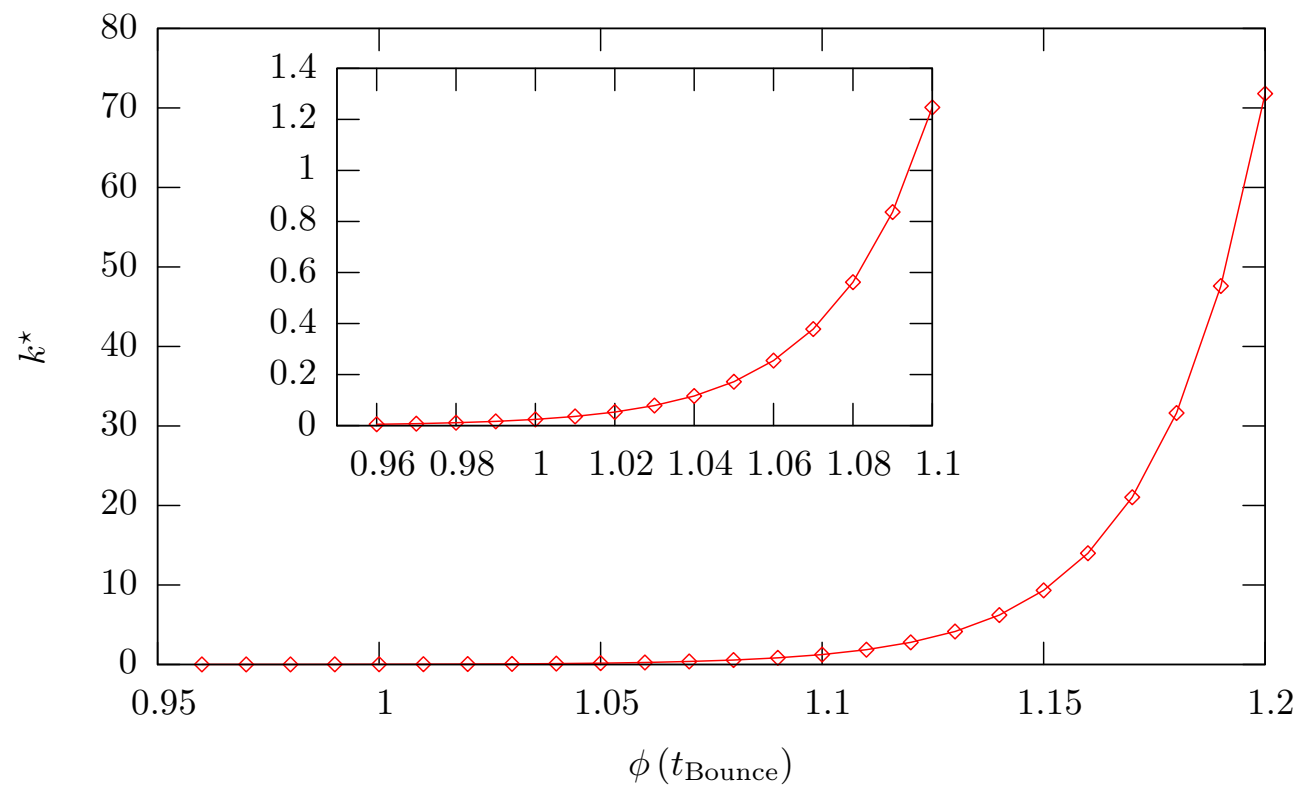

FIG. 3: The value of $k_{\star}$ as a function of $\phi_{\mathrm{B}}$. As the inset shows, $k_{\star}$ always increases with $\phi_{\mathrm{B}}$ but the increase is especially pronounced for $\phi_{\mathrm{B}}>1.1$.

\begin{tabular}{|c|c|c|c|c|}
\hline$\phi\left(\tilde{t}_{B}\right)$ & $k_{\star}$ & $\lambda_{\star}\left(\tilde{t}_{B}\right)$ & $\tilde{t}_{k_{\star}}$ & $\ln \left[a\left(\tilde{t}_{k_{\star}}\right) / a\left(\tilde{t}_{B}\right)\right]$ \\
\hline \hline 0.934 & 0.0016 & 4008 & $1.8 \times 10^{5}$ & 5.2 \\
\hline 1 & 0.024 & 261 & $5.2 \times 10^{5}$ & 8.0 \\
\hline 1.025 & 0.063 & 98.8 & $6.4 \times 10^{5}$ & 9 \\
\hline 1.05 & 0.17 & 37.1 & $7.6 \times 10^{5}$ & 10 \\
\hline 1.075 & 0.45 & 13.8 & $8.8 \times 10^{5}$ & 11 \\
\hline 1.1 & 1.2 & 5.1 & $1.0 \times 10^{6}$ & 12 \\
\hline 1.15 & 9.17 & 0.63 & $1.25 \times 10^{6}$ & 13.9 \\
\hline 1.2 & 70.7 & 0.09 & $1.48 \times 10^{6}$ & 16 \\
\hline 1.3 & $4.58 \times 10^{3}$ & $1.36 \times 10^{-3}$ & $1.97 \times 10^{6}$ & 20.2 \\
\hline 1.5 & $2.7 \times 10^{7}$ & $2.3 \times 10^{-7}$ & $2.9 \times 10^{6}$ & 28.9 \\
\hline
\end{tabular}

TABLE I: This table shows the value of the reference co-moving momentum $k_{\star}$ used in the WMAP data, the corresponding physical wavelength $\lambda_{\star}\left(\tilde{t}_{\mathrm{B}}\right)$ at the bounce, the time $\tilde{t}\left(k_{\star}\right)$ at which the mode $k_{\star}$ exits the Hubble radius during inflation, and $\ln \left[\tilde{a}\left(\tilde{t}\left(k_{\star}\right) / \tilde{a}\left(\tilde{t}_{\mathrm{B}}\right)\right]\right.$, the number of e-folds of expansion between the bounce and $\tilde{t}\left(k_{\star}\right)$. We focus on the range for $\phi_{\mathrm{B}}$ that is relevant to explore whether pre-inflationary dynamics can lead to deviations from the BD vacuum at the onset of the slow roll.

physical wave length $\lambda_{\star}$ of the mode $k_{\star}$ at the bounce point and the number of e-foldings between the bounce, $\tilde{t}=0$ and $\tilde{t}=\tilde{t}\left(k_{\star}\right)$. Results for an interesting range of values of $\phi_{\mathrm{B}}$ are collected in Table 1 .

Finally, we can use Table 1 to analyze the effect of the pre-inflationary dynamics on the initial state of perturbations at the onset of inflation. The argument is semi-heuristic but 
its main conclusions provide reliable guidelines because it is based on rather simple physical considerations. At the bounce, the value of the background scalar curvature is universal, $\tilde{R}_{\mathrm{B}}=62$. This provides a new energy scale $k_{\mathrm{LQC}}$ and the associated wave-length $\lambda_{\mathrm{LQC}}$ :

$$
k_{\mathrm{LQC}}=\left(\frac{62}{6}\right)^{\frac{1}{2}} \approx 3.21, \quad \text { and } \quad \lambda_{\mathrm{LQC}}=\frac{2 \pi}{k_{\mathrm{LQC}}} \approx 0.977,
$$

(see Eq. (4.1) for the origin of the factor 6 and e.g. [75] for a discussion of the observational consequences of states with preferred scales). Since $\left.\tilde{a}\right|_{\mathrm{B}}=1, \quad k_{\mathrm{LQC}}$ and $\lambda_{\mathrm{LQC}}$ are physical energy and length scales at the bounce time. Thus, modes whose physical wave-length is larger than $\lambda_{\mathrm{LQC}}$ at the bounce will experience curvature sometime during their evolution and, as Fig. 1 shows, this will occur in a short time interval soon after the bounce. Will any of these modes be in the observable range for CMB? Recall that, at the surface of last scattering, the physical wave length $\lambda_{\star}$ of the reference mode $k_{\star}$ is about $(1 / 8.58) \times \mathfrak{R}_{\mathrm{LS}}$ where $\mathfrak{R}_{\mathrm{LS}}$ is the radius of the observable universe at that time. Therefore, we will have modes which are both observable in the $\mathrm{CMB}$, and which will experience curvature during their pre-inflationary evolution provided the value of $\phi_{\mathrm{B}}$ is such that

$$
\lambda_{\star} \geq \frac{\lambda_{\mathrm{LQC}}}{8.58} \approx 0.11 .
$$

Table 1 shows that this is possible only if $\phi_{\mathrm{B}}<1.2$. If $\phi_{\mathrm{B}} \gg 1.2$, none of the modes that are in the observational range will encounter significant curvature during their pre-inflationary evolution. Therefore, the initial 4 th order adiabatic vacuum - say, $\left|0^{\text {obv }}\right\rangle$ discussed in section III B, for definiteness - we start out with, will not be excited in these modes. Therefore the state $\left|0^{\text {obv }}\right\rangle$ we constructed at the bounce will be indistinguishable from the obvious 4th adiabatic order vacuum constructed at the onset of inflation. But since the physical wave lengths of the observable modes are all within the Hubble radius at the onset of slow roll, it turns out that the $\mathrm{BD}$ vacuum is observationally indistinguishable from the obvious 4 th adiabatic order vacuum defined at the onset of inflation. Thus, the net result is that $\left|0^{\text {obv }}\right\rangle$ we started out at the bounce will have negligible excitations over the $\mathrm{BD}$ vacuum $|\mathrm{BD}\rangle$ at the onset of inflation in the observable modes. If, on the other hand, $\phi_{B}<1.2$, the state at the onset of inflation would carry excitation over $|\mathrm{BD}\rangle$ in modes that are observable in the CMB and this can serve as a source of new effects $[12,15-17]$. This is why we have focused on kinetic energy dominated bounces.

Of course this conclusion can only be taken as a general guideline. Detailed calculations are necessary to verify that they did not miss a subtle but important point and to sharpen the conclusions through precise quantitative results. In particular, there is no a priori guarantee that the power spectrum will be compatible with the WMAP observations for any value of $\phi_{\mathrm{B}}$. Nor is it clear that the underlying truncation scheme is self-consistent, i.e., that the back reaction of the quantum perturbations can indeed be neglected all the way from the bounce till the onset of slow roll. Section V addresses the first issue, and section VI, the second.

\section{PRE-INFLATIONARY DYNAMICS OF PERTURBATIONS}

In this section we will summarize the results of numerical evolution of quantum fields representing scalar and tensor perturbations all the way from the bounce to the end of slow 
roll inflation. We will then calculate the scalar and tensor power spectra and compare them with those of the standard inflationary scenario. This discussion will provide a conceptual completion of the scenario to include the Planck regime. In addition, we will find that the pre-inflationary dynamics can provide corrections to the standard inflationary scenario with potentially observable consequences.

This section is organized as follows. In $\mathrm{VA}$ we first recall the WMAP phenomenology and then summarize the modifications that arise if the state is not the BD vacuum. In $\mathrm{VB}$ we sketch the plan of the calculations. Illustrative choices of initial conditions are discussed in VC and the results of numerical evolution are presented in VD. Section VE discusses a sample of checks that were performed on the numerics and VF summarizes the overall picture. In broad terms, detailed numerical calculations bear out the physical scenario developed in section IV B and provide detailed quantitative information on the scalar and tensor power spectra in LQC and their relation to those in the standard inflation.

\section{A. WMAP: interplay between theory and observations}

As noted in section III A, the parametrization of the WMAP data is normally carried out assuming that the state of perturbations at the onset of inflation is the BD vacuum. We will start with this parametrization. For values of $\phi_{\mathrm{B}}$ that lead to the BD vacuum at the onset, as in the standard theory, we will have a self-consistent analysis. When there are significant departures from the BD vacuum, one has to revisit the issue and find a parametrization that is consistent with the new quantum state at the onset.

Let us begin with the scalar perturbations. To compare with observations, one generally calculates the power spectrum $\mathcal{P}_{\mathcal{R}}(k)$ of co-moving curvature perturbations $\mathcal{R}_{\vec{k}}$ (see Appendix A). This is because they are conserved to an excellent approximation once the mode exits the Hubble radius during the slow roll: $\mathcal{R}_{\vec{k}}^{\prime} \rightarrow 0$ for $k / a<H$. This insensitivity to the details of the post-inflation dynamics of the background geometry greatly facilitates the task of relating the spectrum of perturbations at the end of inflation with the CMB temperature fluctuations. It is therefore tempting to carry out the entire analysis starting from the bounce using $\mathcal{R}_{\vec{k}}$. Unfortunately, these first order gauge invariant perturbations fail to be well-defined at the 'turn around' during the inflaton evolution where $p_{(\phi)}=0$. On the other hand, the Mukhanov-Sasaki variables $\mathcal{Q}_{\vec{k}}$ introduced in section II are well defined throughout the evolution from the bounce to the end of inflation. Therefore, we numerically evolve $\mathcal{Q}_{\vec{k}}$ starting from the bounce, calculate the power spectrum $\mathcal{P}_{\mathcal{Q}}(k)$, and convert it to the power spectrum $\mathcal{P}_{\mathcal{R}}(k)$ at the end of inflation via

$$
\mathcal{P}_{\mathcal{Q}}(k)=\left|\frac{z}{a}\right|^{2} \mathcal{P}_{\mathcal{R}}(k), \quad \text { where } \quad z(\tilde{\eta})=-\frac{6}{\kappa} \frac{p_{(\phi)}}{\pi_{(a)}} \equiv \frac{\gamma}{a^{2} \ell^{3}} \frac{p_{(\phi)}}{\mathrm{b}} .
$$

Given a 4th adiabatic order vacuum $|\Omega\rangle$ of the scalar perturbation, the power spectrum $\mathcal{P}_{\mathcal{Q}}(k)$ for the Mukhanov-Sasaki variables is defined in terms of the associated two point function in the momentum space:

$$
\left\langle\Omega\left|\hat{\mathcal{Q}}_{\vec{k}} \hat{\mathcal{Q}}_{\vec{k}^{\prime}}\right| \Omega\right\rangle:=(2 \pi)^{3} \delta\left(\vec{k}+\vec{k}^{\prime}\right) \frac{2 \pi^{2}}{k^{3}} \mathcal{P}_{\mathcal{Q}}^{\Omega}(k)
$$

where all quantities are evaluated a few e-folds after the time $\tilde{\eta}_{k}$, defined by $k / \tilde{a}\left(\tilde{\eta}_{k}\right)=H\left(\tilde{\eta}_{k}\right)$, at which the mode $k$ 'exits the Hubble radius'. As discussed above, at the onset of the slow 
roll we will first set $|\Omega\rangle=|\mathrm{BD}\rangle$, the $\mathrm{BD}$ vacuum, and introduce the modifications that are needed for a general state $|\Omega\rangle$ at the end. As is well-known, a basis $q_{k}^{\mathrm{BD}}(\tilde{\eta})$ that defines $|\mathrm{BD}\rangle$ is given by

$$
q_{k}^{\mathrm{BD}}(\tilde{\eta})=\frac{1}{\tilde{a}} \sqrt{\frac{-\tilde{\eta} \pi}{4}} H_{\mu}^{(1)}(-k \tilde{\eta}),
$$

where $H_{\mu}^{(1)}(-k \tilde{\eta})$ is a Hankel function (of the first kind). The index $\mu$ is determined by the slow roll parameters, $\mu=3 / 2+2 \epsilon+\delta$, with

$$
\epsilon:=-\frac{\dot{H}}{H^{2}} \approx \frac{1}{2 \kappa}\left(\frac{V_{, \phi}}{V}\right)^{2} \quad \text { and } \quad \delta:=\frac{\ddot{H}}{2 \dot{H} H} \approx \frac{1}{\kappa}\left[\left(\frac{1}{2} \frac{V_{, \phi}}{V}\right)^{2}-\left(\frac{V_{, \phi \phi}}{V}\right)\right] .
$$

Here the 'dot' refers to the derivative with respect to the cosmic time $\tilde{t}$ and we have ignored quantities quadratic in slow roll parameters to approximate them by functions of the potential $V(\phi)$. The mode functions $q_{k}(\tilde{\eta})$ determine the power spectrum via $\mathcal{P}_{\mathcal{Q}}^{\mathrm{BD}}(k)=\hbar\left(k^{3} / 2 \pi^{2}\right)\left|q_{k}^{\mathrm{BD}}\right|^{2}$, with the modes evaluated a few e-folds after $\tilde{\eta}_{k}$. Using the asymptotic properties of Hankel functions, the background equations and the relation between $\mathcal{P}_{\mathcal{Q}}^{\mathrm{BD}}(k)$ and $\mathcal{P}_{\mathcal{R}}^{\mathrm{BD}}(k)$, one arrives at well-known expression ${ }^{10}$,

$$
\mathcal{P}_{\mathcal{R}}^{\mathrm{BD}}(k)=\frac{\hbar \kappa}{2 \epsilon\left(\tilde{\eta}_{k}\right)}\left(\frac{H\left(\tilde{\eta}_{k}\right)}{2 \pi}\right)^{2},
$$

where, on the right side, the $k$-dependence is encoded in the time $\tilde{\eta}_{k}$ at which $H$ and $\epsilon$ are evaluated. Since $H$ and $\epsilon$ change very slowly during slow roll, $\mathcal{P}_{\mathcal{R}}^{\mathrm{BD}}(k)$ depends very weakly on $k$; it is almost scale-invariant. The weak $k$ dependence is parameterized by the scalar spectral index $n_{s}(k)$,

$$
n_{s}^{\mathrm{BD}}(k)-1:=\frac{d \ln \mathcal{P}_{s}^{\mathrm{BD}}(k)}{d \ln k}=-2\left(2 \epsilon\left(\tilde{\eta}_{k}\right)+\delta\left(\tilde{\eta}_{k}\right)\right) .
$$

(Note that in Eqs. (5.3), (5.5) and (5.6), one ignores terms which are quadratic and higher order in slow roll parameters.) The WMAP observations [72] provide

$$
\mathcal{P}_{\mathcal{R}}\left(k_{\star}\right)=(2.430 \pm 0.091) \times 10^{-9} \quad \text { and } \quad n_{s}\left(k_{\star}\right)=0.968 \pm 0.012 ，
$$

where $k_{\star}$ is the WMAP reference mode introduced before. For the potentials $V(\phi)$ commonly employed in inflation (quadratic, quartic, exponential, etc.), the parameters $\epsilon$ and $\delta$ are not independent. For the case of a quadratic potential considered here, $\delta=0$, and observational data (5.7) gives the values reported in (3.2)

$$
H\left(\tilde{\eta}\left(k_{\star}\right)\right)=7.83 \times 10^{-6} \text { and } \epsilon\left(\tilde{\eta}\left(k_{*}\right)\right)=8 \times 10^{-3} .
$$

To conclude this sub-section, let us discuss the modifications that occur if the quantum state of the scalar perturbation is $|\Omega\rangle \neq|\mathrm{BD}\rangle$. In view of the initial conditions discussed in section III, $|\Omega\rangle$ can be taken to be a 'vacuum' which is determined by a 4 th adiabatic order basis $q_{k}(\tilde{\eta})$ which differs from $q_{k}^{\mathrm{BD}}(\tilde{\eta})$ :

$$
q_{k}(\tilde{\eta})=\alpha_{k} q_{k}^{B D}(\tilde{\eta})+\beta_{k} q_{k}^{B D \star}(\tilde{\eta}) .
$$

${ }^{10}$ In the cosmological literature it is common to set $\hbar=1$ but retain and express $\kappa$ in terms of the 'reduced' Planck mass $M_{P l}^{2}=\hbar / \kappa$. 
Here, the Bogoliubov coefficients $\alpha_{k}$ and $\beta_{k}$ are time independent and are functions only of $k:=|\vec{k}|$. Since the basis $q_{k}(\tilde{\eta})$ is also normalized, we have $\left|\alpha_{k}\right|^{2}-\left|\beta_{k}\right|^{2}=1$. Physically, $\left|\beta_{k}\right|^{2}$ represents the number density of the BD 'excitations' with momentum $\vec{k}$, per unit co-moving volume in position as well as momentum space, contained in the state $|\Omega\rangle$. The power spectrum and the spectral index determined by $|\Omega\rangle$ are given by

$$
\begin{aligned}
\mathcal{P}_{\mathcal{R}}^{\Omega}(k) & =\mathcal{P}_{\mathcal{R}}^{\mathrm{BD}}(k)\left|\alpha_{k}+\beta_{k}\right|^{2}, \\
n_{s}^{\Omega}(k)-1 & =n_{s}(k)^{B D}-1+\frac{d \ln \left|\alpha_{k}+\beta_{k}\right|^{2}}{d \ln k} .
\end{aligned}
$$

If the coefficients $\alpha_{k}$ and $\beta_{k}$ are not trivial, i.e. $\beta_{k}$ differ significantly from zero, our procedure will fail to be self-consistent because the background quantities extracted from observation will differ from (3.2), the values assumed at the start of our numerical evolution. We then have to seek a new solution. In the detailed numerical simulations we have carried out so far - with $\phi_{\mathrm{B}} \geq 1.15$ - we find that even when the state $|\Omega\rangle$ at the onset of the slow roll differs from the $\mathrm{BD}$ vacuum, the difference is sufficiently small for the parametrization of the data using the $\mathrm{BD}$ vacuum to be adequate.

Remark: If $\phi_{\mathrm{B}}$ is significantly less than 1.15 , the $\mathrm{BD}$ parametrization will not be adequate. In these cases, one can use a simple 'cyclic method'. As before, one can start with the inflaton mass (3.1) obtained from the BD vacuum and calculate the quantum state $|\Omega\rangle$ at the onset of inflation. If this state differs significantly from the $\mathrm{BD}$ vacuum, i.e. if the $\beta_{k}$ coefficients are large, one can recalculate the inflaton mass using background quantities at time $\tilde{\eta}\left(k_{\star}\right)$ obtained from (5.9). One can then use this value of the inflaton mass and recalculate the state at the onset of inflation. If this state $\left|\Omega_{1}\right\rangle$ resulting from this first iteration agrees with $|\Omega\rangle$ we have a self consistent solution. If not, one has to continue the iteration procedure until there is self consistency. Of course, there is no a priori guarantee that this iterative procedure will converge. However, details of the pre-inflationary LQC dynamics summarized in section IV A suggest that not only will it converge, but the convergence may be reached just after the first iteration. Recall that: i) we are interested in only those modes which are observable in the CMB, and, ii) only the modes whose physical wave lengths exceed the curvature radius during evolution have $\mathrm{BD}$ excitations at the onset of inflation. As we saw in section IV A, this circumstance occurs only during a short interval close to the bounce (see the right panel in Fig. 1). At that time, the background is dominated by quantum-geometry effects, which are (approximately) universal, i.e., largely insensitive to the value of the mass $m$ in the potential $V(\phi)$. Therefore, after a change in the value of $m$ as a consequence of the first iteration, one does not expect a significant change to the number of created quanta. Hence, the coefficients $\alpha_{k}$ and $\beta_{k}$ should remain (approximately) unchanged. This argument is supported by a few preliminary numerical computations for $0.93 \leq \phi_{\mathrm{B}}<1.15$ where one obtains convergence after one iteration. These low $\phi_{\mathrm{B}}$ appear to exhibit a number of interesting features that are relevant to non-Gaussianities which will be discussed in detail in a separate publication.

\section{B. Plan of the calculations}

The scalar and tensor perturbations $\hat{\mathcal{Q}}_{\vec{k}}, \hat{\mathcal{T}}_{\vec{k}}$ propagate on the quantum corrected, effective solution $\tilde{a}(\tilde{t})$ and $\phi(\tilde{t})$, satisfying: 


$$
\begin{array}{r}
\ddot{\hat{\mathcal{Q}}}_{\vec{k}}+3 H \dot{\hat{\mathcal{Q}}}_{\vec{k}}+\frac{\left(k^{2}+\tilde{\mathfrak{A}}\right)}{\tilde{a}^{2}} \hat{\mathcal{Q}}_{\vec{k}}=0, \\
\ddot{\mathcal{T}}_{\vec{k}}+3 H \dot{\mathcal{T}}_{\vec{k}}+\frac{k^{2}}{\tilde{a}^{2}} \hat{\mathcal{T}}_{\vec{k}}=0,
\end{array}
$$

where, as usual, the 'dot' denotes derivative with respect to the cosmic $\tilde{t}, H=\dot{\tilde{a}} / \tilde{a}$ is the Hubble parameter and $\tilde{\mathfrak{A}}$ is defined in (2.14). As discussed in section III, we will assume that the quantum state of perturbations is a vacuum $|\Omega\rangle$ of 4 th adiabatic order. We provide concrete examples of these states in section VC. Each of these states is determined by a basis which we will denote by $q_{k}$ for scalar perturbations and by $e_{k}$ for tensor perturbations. They satisfy (5.10) and (5.11) respectively and are normalized:

$$
\begin{aligned}
q_{k}(\tilde{t}) \dot{q}_{k}^{\star}(\tilde{t})-\dot{q}_{k}(\tilde{t}) q_{k}^{\star}(\tilde{t}) & =\frac{i}{\tilde{a}^{3}}, \\
e_{k}(\tilde{t}) \dot{e}_{k}^{\star}(\tilde{t})-\dot{e}_{k}(\tilde{t}) e_{k}^{\star}(\tilde{t}) & =\frac{4 i \kappa}{\tilde{a}^{3}}
\end{aligned}
$$

Then, as discussed in section VA, the power spectra are given by

$$
\mathcal{P}_{\mathcal{T}}(k)=\hbar \frac{k^{3}}{2 \pi^{2}}\left|e_{k}\right|^{2} \quad \text { and } \quad \mathcal{P}_{\mathcal{R}}(k)=\hbar \frac{k^{3}}{2 \pi^{2}}\left(\frac{\dot{\phi}}{H}\right)^{2}\left|q_{k}\right|^{2},
$$

where the mode functions are evaluated at the end of inflation. Thus, to obtain the power spectra, we need to evolve the two sets of bases functions. Numerical calculations were carried out and we will present plots for both scalar and tensor perturbations. However, for brevity we will focus on the scalar modes in most of our narrative.

To bring out the similarities and differences between the chosen 4th adiabatic order vacuum $|\Omega\rangle$ and the $\mathrm{BD}$ vacuum $|\mathrm{BD}\rangle$ we will also compute the Bogoliubov coefficients $\alpha_{k}$ and $\beta_{k}$ relating the two vacua, defined in (5.8). Using this relation, its time derivative and the normalization condition, it is straightforward to obtain expressions for $\alpha_{k}$ and $\beta_{k}$ :

$$
\begin{aligned}
\alpha_{k} & =i \tilde{a}^{3}(\tilde{t})\left[\dot{q}_{k}(\tilde{t}) q_{k}^{\mathrm{BD} \star}(\tilde{t})-q_{k}(\tilde{t}) \dot{q}_{k}^{\mathrm{BD} \star}(\tilde{t})\right], \\
\beta_{k} & =-i \tilde{a}^{3}(\tilde{t})\left[\dot{q}_{k}(\tilde{t}) q_{k}^{\mathrm{BD}}(\tilde{t})-q_{k}(\tilde{t}) \dot{q}_{k}^{\mathrm{BD}}(\tilde{t})\right] .
\end{aligned}
$$

Note that although the right sides of (5.14) contain time-dependent terms, $\alpha_{k}, \beta_{k}$ themselves are time independent and they satisfy the identity $\left|\alpha_{k}\right|^{2}-\left|\beta_{k}\right|^{2}=1$. These properties will be used to monitor the numerical accuracy of our simulations.

The numerical evolutions were performed using the internal Mathematica numerical integrators (adaptive $4^{\text {th }}$ order Runge-Kutta).

\section{Illustrative examples of states}

As discussed in section III B, the quantum states $|\Omega\rangle$ of interest are vacua of 4 th adiabatic order which satisfy an additional initial condition: At the bounce time $\tilde{t}=0$, the renormalized energy density in the state $|\Omega\rangle$ is negligible compared to the universal energy density $\rho_{\max }$ in the background. These conditions allow an infinite class of states $|\Omega\rangle$. However, in each numerical simulation we need to work with a specific choice.

Our detailed simulations were carried out using the state $|\Omega\rangle=\left|0^{\text {obv }}\right\rangle$ which was defined in section IV B. As discussed there, the state is rather simple to construct. The definition 
is tied to an instant of time $\tilde{t}$ and is 'local' in the sense that it is sensitive only to the scale factor $\tilde{a}$ and its first four time derivative evaluated at $\tilde{t}$. Consider a mathematical example in which $\tilde{a}$ is constant during small time intervals around times $\tilde{t}_{1}$ and $\tilde{t}_{2}$. In this case, the prescription would lead to the standard vacuum in Minkowski space-time at these two times and the 'particle creation' from $\tilde{t}_{1}$ to $\tilde{t}_{2}$ would be exactly as in Parker's original work of $[68,70]$ which initiated the study of quantum fields in cosmological space-times. As a second example, consider the case in which the $\tilde{t}$ derivative of $H=\dot{a} / a$ vanishes in an small interval around a time $\tilde{t}_{o}$. Then the state would be indistinguishable from the $\mathrm{BD}$ vacuum for high frequency modes, i.e., modes for which terms of the order $\mathcal{O}\left((k / H \tilde{a})^{6}\right)$ can be neglected. Finally, as explained in section IV B, $\left|0^{\text {obv }}\right\rangle$ has the attractive feature that, in a precise sense, it minimizes the uncertainties in the fundamental canonically conjugate fields at an instant of time. These properties motivated our use of the 'obvious vacuum', tailored to the bounce time, in detailed numerical calculations.

However, to develop intuition for whether the main results are sensitive to the specific choice of state, we also carried out several simulations using three other states. We will summarize these choices both for completeness and because some of them may be useful in future investigations.

- Zero energy-state at the bounce: In the absence of a larger group of isometries to single out a preferred quantum state, we can further constrain the family of 4thorder adiabatic vacua by imposing additional physical conditions. Considering the key role played by back-reaction in our truncation scheme, one natural requirement is to ask that the initial state have vanishing expectation value of the renormalized energy density. In the context of adiabatic regularization it is possible to construct 4 th adiabatic order states with this property at any given time [76]. These states are tailored to a given time, in the sense that their energy density is not zero at later times. Although this condition does not select a unique 4th-order vacuum, it narrows down considerably the possibilities.

- Zero stress-energy state at the bounce: A stronger prescription to select a natural vacuum state is to demand that the state have vanishing expectation value of the renormalized energy-momentum tensor at a given time. When the infra-red cut-off in the momentum integrals is sufficiently large, this selects a unique state at the bounce [76]. However, for smaller cut-offs (and general times), this state does not exist. As in the case of the obvious 4th order adiabatic vacuum at the bounce (see subsection IIIB), for those cases some of the modes defining that state need to be modified. This introduces a freedom in the definition and the resulting state no longer has zero expectation value of the energy-momentum tensor. Nevertheless, this prescription is useful, in particular when the cut-off is sufficiently large (i.e. $\phi_{B}$ is sufficiently large).

- It follows from our discussion in the beginning of section IV A that, if we re-scale tensor modes via $\chi_{k}=\tilde{a} e_{k}$, then $\chi_{k}$ can be regarded as modes propagating in Minkowski space but with 'time dependent frequency' $w(\tilde{\eta})$. (This statement extends also to the scalar modes.) Following the procedure used in Minkowski space to construct the preferred vacuum state, one can imagine using 'instantaneous positive frequency modes' at a given time, with frequency $w(\tilde{\eta})$. However, this naive choice needs to be modified both for low and high $k$. For low $k$ the reason is that the 'frequency' $w(\tilde{\eta})$ becomes imaginary. For large $k$ the frequency is positive, however the modes do 
not define a 4th adiabatic order state and hence, if (for example) the energy density is to be well defined, at large $k$ the prescription needs to be changed. While there is large freedom in those modifications, if one restricts oneself to observable modes, the freedom becomes largely irrelevant. Therefore, this methods is well suited for quick calculations of power spectra and provides a useful way to analyze the effects of different initial conditions.

In all these three cases, we found that the main features of the power spectra and the energy density in the perturbations were the same as those calculated with $\left|0^{\text {obv }}\right\rangle$ and reported sections VD and VI. In this sense the results are robust and not tied to the choice $|\Omega\rangle=\left|0^{\mathrm{obc}}\right\rangle$.

\section{Numerical evolution}

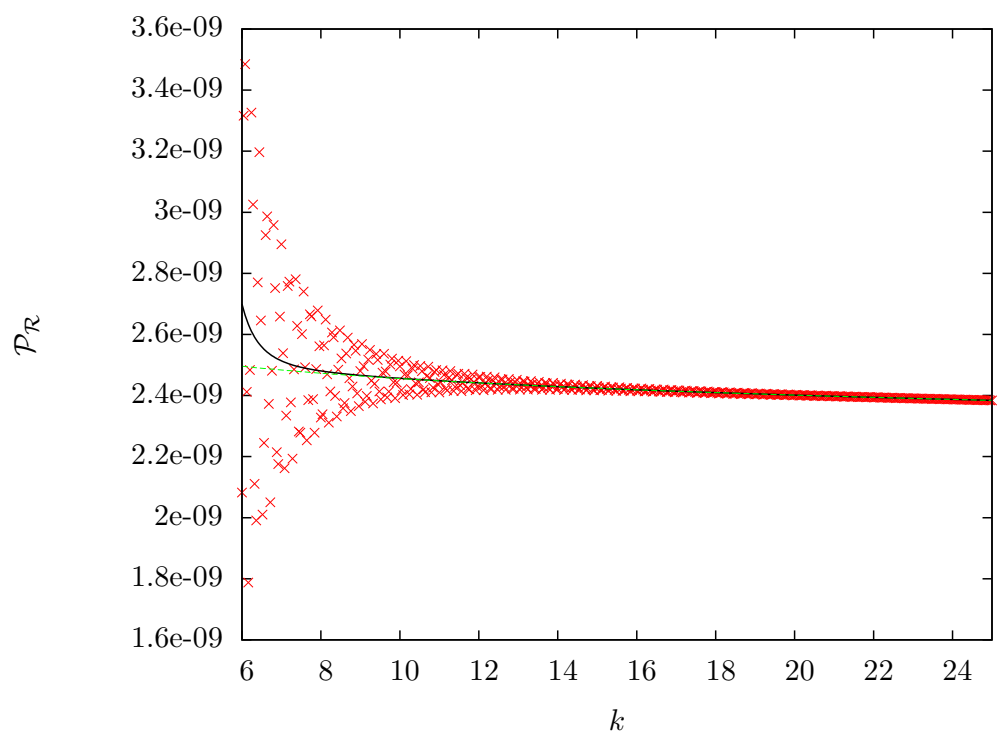

FIG. 4: Power-spectrum for the scalar perturbations (crosses) for $\phi_{\mathrm{B}}=1.15$. The solid line shows the average of the points and the (green) dashed line is the standard inflationary power-spectrum which assumes the BD vacuum at the onset of smooth roll. The deviation from the Bunch-Davies power-spectrum at low $k$ is not numerical noise. Rather, modes with these frequencies experience background curvature during their pre-inflationary evolution and are thus excited. The state $|\Omega\rangle$ contains excitations over the $\mathrm{BD}$ vacuum $|\mathrm{BD}\rangle$ with quanta of these modes. This leads to a highly oscillatory power spectrum discussed in the text.

Recall that the parameter space for the background is dictated by the value $\phi_{\mathrm{B}}$ assumed by the background inflaton at the bounce. Ideally one would like to explore as much of this parameter space as possible. However, because of the exponential relationship between $\phi_{B}$ and $\tilde{a}\left(\tilde{t}\left(k_{\star}\right)\right)$ (see Tab. I and Fig. 3), even a relatively modest value of $\phi_{B}$ results in extremely long integration times, which rapidly becomes computationally prohibitive. Therefore, we have had to restrict our attention to $\phi_{B} \lesssim 2.0$. Fortunately, as we discussed in section IV B, this interval covers the region of the parameter space that is physically most interesting. For, 
our numerical simulations bear out the expectation based on those physical considerations: the main results are essentially insensitive to the value of $\phi_{\mathrm{B}}$ once $\phi_{\mathrm{B}}$ exceeds 1.2.

From an observational point of view, the most significant result is the scalar powerspectrum, plotted in Fig. 4, for $\phi_{B}=1.15$ and the 'obvious' 4th adiabatic order vacuum, i.e. for $|\Omega\rangle=\left|0^{\text {obv }}\right\rangle$. We will now comment on various features of the plot.

First, the physical argument in section IV B suggested that modes with $k \gg k_{\mathrm{LQC}} \sim 3.21$ will not be excited because their physical wave length would be smaller than the curvature radius throughout the pre-inflationary evolution. Therefore, these modes would be in the $\mathrm{BD}$ vacuum at the onset of inflation. This reasoning is borne out because for large $k$ the LQC power spectrum for these modes with $|\Omega\rangle=\left|0^{\text {obv }}\right\rangle$ as the initial state at the bounce is essentially the same as that obtained in the standard inflationary scenario with $|\mathrm{BD}\rangle$ as the state at the onset of inflation. Second, for modes with lower $k$ values, the LQC power spectrum shows a highly oscillatory behavior. This phenomenon has been noted before (see e.g. $[8,77,78])$. Its origin can be traced back to Eq. (5.9):

$$
P_{\mathcal{R}}^{\Omega}(k)=P_{\mathcal{R}}^{\mathrm{BD}}(k)\left|\alpha_{k}+\beta_{k}\right|^{2}=P_{\mathcal{R}}^{\mathrm{BD}}(k)\left(1+2\left|\beta_{k}\right|^{2}+2 \mathbb{R} e\left(\alpha_{k} \beta_{k}^{\star}\right)\right),
$$

where in the second equality we have used the normalization condition $\left|\alpha_{k}\right|^{2}-\left|\beta_{k}\right|^{2}=1$. The oscillatory behavior of the power-spectra arises from the final interference term, due to the rapidly changing relative phase. This oscillation is so fast in $k$ that in any realistic observations -which have a finite $k$ resolution- they would be 'averaged out'. Therefore, in [18] we introduced 'bins' in the $k$ space with a width $0.5 \ell_{\mathrm{Pl}}^{-1}$ and averaged the oscillations in each bin. It turns out that the resulting plot is indistinguishable from the one obtained by simply neglecting the interference term in Eq. (5.15). If we do so, the LQC power spectrum $P_{\mathcal{R}}^{\Omega}(k)$ is given simply by rescaling $P_{\mathcal{R}}^{\mathrm{BD}}(k)$ with $\left(1+2\left|\beta_{k}\right|^{2}\right)$. Since the number density of excitations in the mode $k$ is given by $\left|\beta_{k}\right|^{2}$, this simplified version of Eq. (5.15) brings to forefront the fact that the modification of the power spectrum can be traced back directly to the creation of excitations by curvature. The number of excitations decay rapidly as $k$ increases because modes with smaller wave lengths do not experience as much curvature during their pre-inflationary evolution. Hence the $\beta_{k}$ coefficients decay rapidly with $k$, damping the amplitude of the oscillations.

The relation between the LQC and the BD power spectra is brought out more clearly in Fig. 5 where we plot the ratio $P_{\mathcal{R}}^{\Omega}(k) / P_{\mathcal{R}}^{B D}(k)$. To define the $\mathrm{BD}$ vacuum, mode functions were not approximated by Hankle functions for all times. Rather they were taken to be Hankle functions at a time when the physical frequency of the mode $k_{\star}$ was $10^{3} \mathrm{H}^{-1}$, to ensure that all the modes of interest were well inside the Hubble radius, and then evolved numerically. The circles represent the actual data points which show oscillations while the solid (red) curve is the plot without oscillations (or, obtained by binning as discussed above). Since $\phi_{B}=1.15$, it follows from Table 1 that the reference mode used in the WMAP data is $k_{\star}=9.17$. It is clear from Fig. 5 that, at this value, the LQC and the standard inflationary predictions are almost indistinguishable. Consequently, although the LQC state at the onset of inflation differs from the standard $\mathrm{BD}$ vacuum for low $k$, nonetheless the LQC prediction is in agreement with the WMAP values (5.7) of the amplitude and spectral index which are evaluated at the time $t\left(k_{\star}\right)$.

So far we focused on the background geometry with $\phi_{\mathrm{B}}=1.15$. What is the situation with other values? The physical considerations of section IV B suggest that if we examine the excitations in any one mode $\vec{k}$-ignoring for the moment the issue of whether it is observable in the CMB - then the LQC power spectrum would not be sensitive to the specific value 


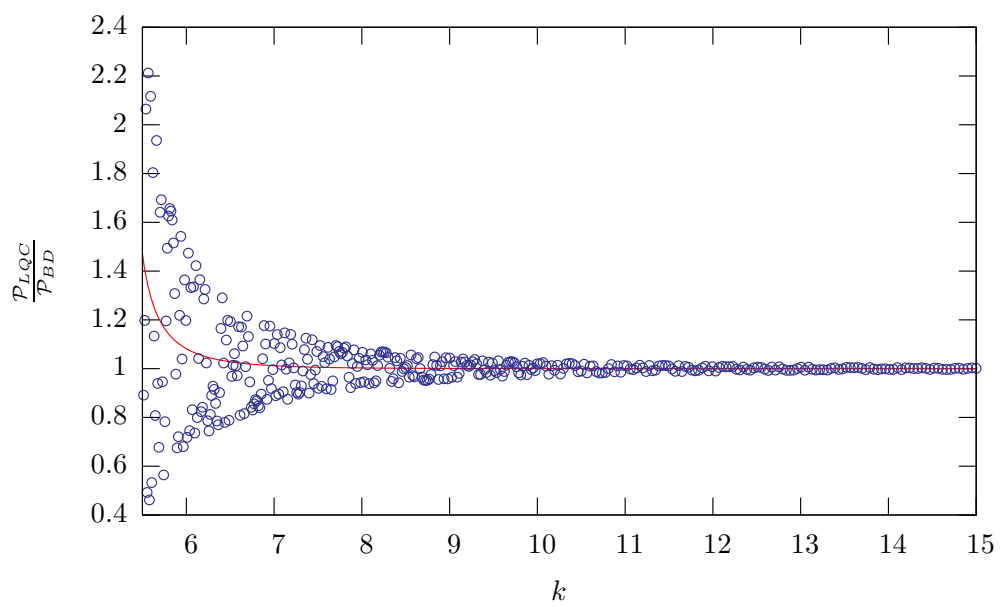

FIG. 5: Ratio of the LQC power spectrum $P_{\mathcal{R}}^{\Omega}(k)$ for scalar perturbations to the standard one, $P_{\mathcal{R}}^{\mathrm{BD}}(k)$ which assumes the BD state at the onset of slow-roll inflation. The circles represent the actual ratio which exhibits rapid oscillations at low $k$ due to a rapidly varying relative phase. The line is the smooth part of this ratio, which can either be produced by binning the exact data, or by removing the interference factor, as explained in the text. This smoothed ratio is simply $1+2 N_{k}$, where $N_{k}$ is the number of $\vec{k}$ particles.

of $\phi_{\mathrm{B}}$. The same should hold for the ratio $P_{\mathcal{R}}^{\Omega}(k) / P_{\mathcal{R}}^{B D}(k)$ since the standard inflationary power spectrum makes no reference to $\phi_{\mathrm{B}}$ at all. Is this expectation borne out? Fig. 6 shows that the answer is in the affirmative. Specifically, we plot the fractional difference between the scalar power-spectra, calculated using different values of $\phi_{B}$. This fractional difference is less than $\approx 0.2 \%$ over the range of $k$ in which there is a significant deviation from the BD power-spectra. The true difference is, in fact, smaller than this, since the value at large $k$ is dominated by numerical error (see Section VE).

These results lead us to the following interesting overall picture. If we look at the entire range of wave numbers $k$, the ratio of the LQC and BD power spectra is essentially universal, i.e., insensitive to the value of $\phi_{\mathrm{B}}$ (at least for the range of $\phi_{\mathrm{B}}$ we analyzed in detail). Modes with $k \gg k_{\mathrm{LQC}}=3.21$ are in the $\mathrm{BD}$ vacuum at the onset of inflation while those with lower values of $k$ are excited because they experience the background curvature in the Planck regime immediately after the bounce. What changes with $\phi_{\mathrm{B}}$ is the window in the $k$ space that is accessible to observations in the $C M B$. As Table 1 shows, each $\phi_{B}$ determines the co-moving value of $k_{\star}$, the reference mode used in by WMAP (consistent with our convention $\left.\left.\tilde{a}\right|_{\tilde{t}=0}=1\right)$. The observationally relevant window is given by $\left(k_{o}, 2000 k_{o}\right)$, where $k_{o}=k_{\star} / 8.58$, and moves rapidly to the right as $\phi_{\mathrm{B}}$ increases.

Next, in Fig. 7 we plot the power-spectrum of the tensor modes. Fig. 7 is very similar to the power spectrum of the scalar modes shown in Fig. 4, even though the evolution equation (5.10) for the scalar mode includes a potential $\mathfrak{A}$ which is absent in the tensor modes. This is because from its definition (2.6), it is clear that the potential is proportional to $m^{2}$, the square of the inflaton mass, and $m^{2} \sim 10^{-12}$. Since the $\mathfrak{f}$ that features in $(2.6)$ ranges between 0 and 1 and, for kinetic dominated bounces, $\phi<10$ during the entire preinflationary evolution, the effects of the external potential $\tilde{\mathfrak{A}}$ turns out to be negligible for the range of $k$ 's of interest to observations. As a consequence, the evolution of the two sets of bases functions is almost indistinguishable: $\left|\beta_{k}^{\mathcal{Q}}\right|^{2} \simeq\left|\beta_{k}^{\mathcal{T}}\right|^{2}$; in the Planck regime, the same 


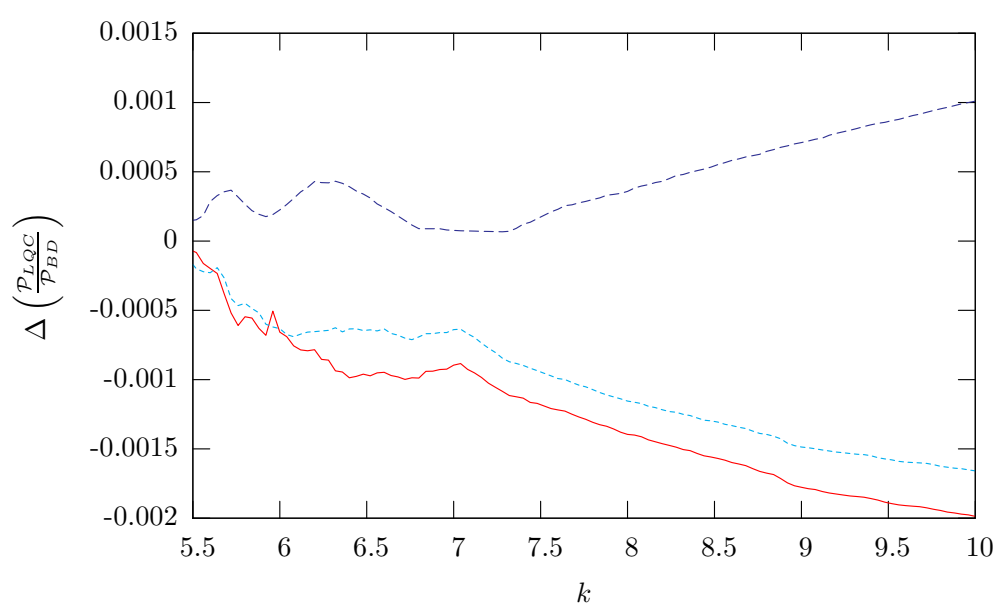

FIG. 6: The ratio of the LQC power spectrum $P_{\mathcal{R}}^{\Omega}(k)$ for scalar perturbations to the standard one, $P_{\mathcal{R}}^{\mathrm{BD}}(k)$, is insensitive to the value of $\phi_{\mathrm{B}}$. Here we have plotted the fractional difference between ratios calculated using different values of $\phi_{B}$, i.e. $\Delta\left(P_{\mathrm{LQC}} / P_{\mathrm{BD}}\right) \equiv\left(\left.\left(P_{\mathrm{LQC}} / P_{\mathrm{BD}}\right)\right|_{\phi_{\mathrm{B}}}-\right.$ $\left.\left.\left(P_{\mathrm{LQC}} / P_{\mathrm{BD}}\right)\right|_{\phi_{\mathrm{B}}=\dot{\phi}}\right)\left(\left.\left(P_{\mathrm{BD}} / P_{\mathrm{LQC}}\right)\right|_{\phi_{\mathrm{B}}=\dot{\phi}}\right)$, where $\dot{\phi}=1.1$ and the solid (red) line is for $\phi_{\mathrm{B}}=1.0$, the dashed (blue) line is for $\phi_{\mathrm{B}}=1.05$ and the dotted (cyan) line is for $\phi_{\mathrm{B}}=1.15$. Note that the deviation from zero is almost entirely due to numerical precision, but even so the ratios are the same to a few parts in $10^{3}$.

amount of quanta are created in tensor and scalar modes. An immediate consequence is that the LQC ratio of the tensor to scalar power spectra, $r_{\mathrm{LQC}}$, (when averaged) is the same as in standard inflation

$$
r_{\mathrm{LQC}}:=\frac{2 P_{\mathcal{T}}^{\Omega}}{P_{\mathcal{R}}^{\Omega}}=\frac{2 P_{\mathcal{T}}^{\mathrm{BD}}\left(1+2\left|\beta_{k}^{\mathcal{T}}\right|^{2}\right)}{P_{\mathcal{R}}^{\mathrm{BD}}\left(1+2\left|\beta_{k}^{\mathcal{Q}}\right|^{2}\right)} \approx \frac{2 P_{\mathcal{T}}^{\mathrm{BD}}(k)}{P_{\mathcal{R}}^{\mathrm{BD}}(k)}=r_{\mathrm{BD}} .
$$

This result is shown in Fig. (8), where we plot the ratio $r_{\mathrm{LQC}} / r_{\mathrm{BD}}$.

To conclude, let us comment on an inflationary consistency relation. An important result of the standard inflationary scenario is the relation between the tensor-to-scalar ratio and the tensor spectral index. In slow roll inflation this relation reads $r_{\mathrm{BD}} \approx-8 n_{t}^{\mathrm{BD}}$, where the approximation indicates that terms of quadratic or higher order in the slow roll parameters are neglected. Since this expression holds independently of the inflaton potential and relates two independent observable quantities, it serves as a test of the standard scenario. Forthcoming observation of the effect of tensor perturbations in the CMB will provide a test of this relation. Does LQC modify this relation? We have seen that the tensor-to-scalar ratio remains unmodified. However, the tensor spectral index $i s$ modified because of the preinflationary evolution. The LQC tensor spectral index is obtained from the tensor power spectrum after averaging

$$
n_{t}=\frac{d \ln P_{\mathcal{T}}^{\Omega}}{\ln k}=\frac{d \ln P_{\mathcal{T}}^{\mathrm{BD}}}{\ln k}+\frac{d \ln \left(1+2\left|\beta_{k}^{\mathcal{T}}\right|^{2}\right)}{d \ln k}=n_{t}^{\mathrm{BD}}-1+\frac{d \ln \left(1+2\left|\beta_{k}^{\mathcal{T}}\right|^{2}\right)}{d \ln k} .
$$

Therefore, the LQC consistency relation is given by

$$
r_{\mathrm{LQC}} \approx-8\left(n_{t}-\frac{d \ln \left(1+2\left|\beta_{k}^{(\mathcal{T})}\right|^{2}\right)}{d \ln k}\right)
$$




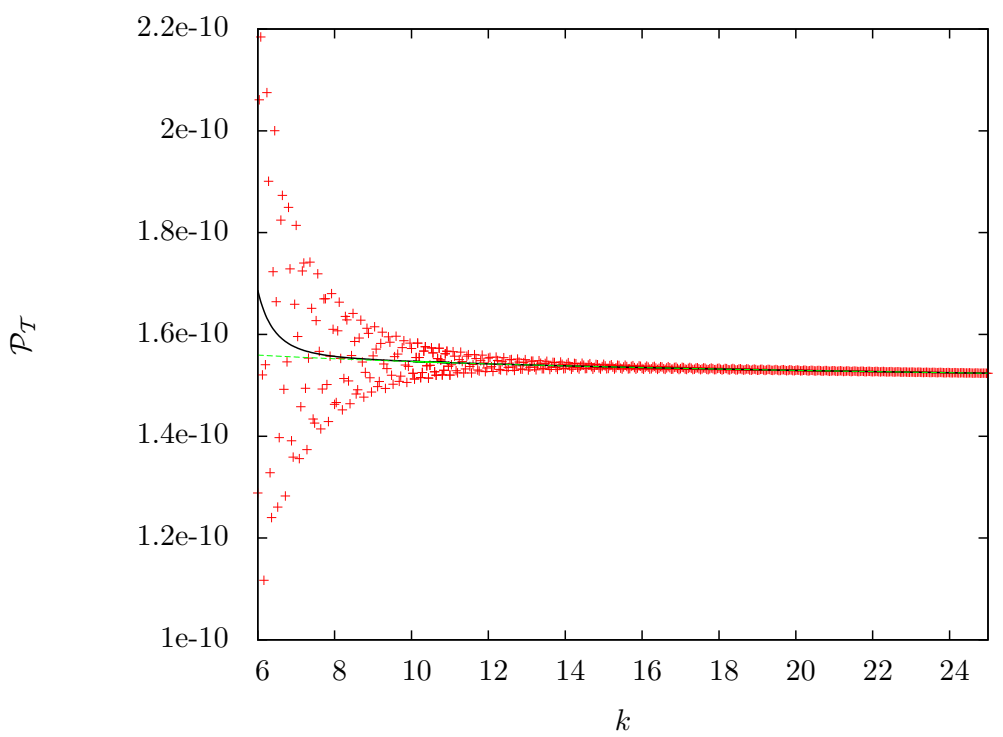

FIG. 7: The LQC power spectrum for tensor modes. As for scalar modes, we have set $\phi_{B}=1.15$ and used the 'obvious' 4th adiabatic order vacuum at the bounce, $|\Omega\rangle=\left|0^{\text {obv }}\right\rangle$ (crosses). The solid line is the average and the dashed line is the standard tensor power-spectrum assuming the BD at the onset of slow-roll inflation.

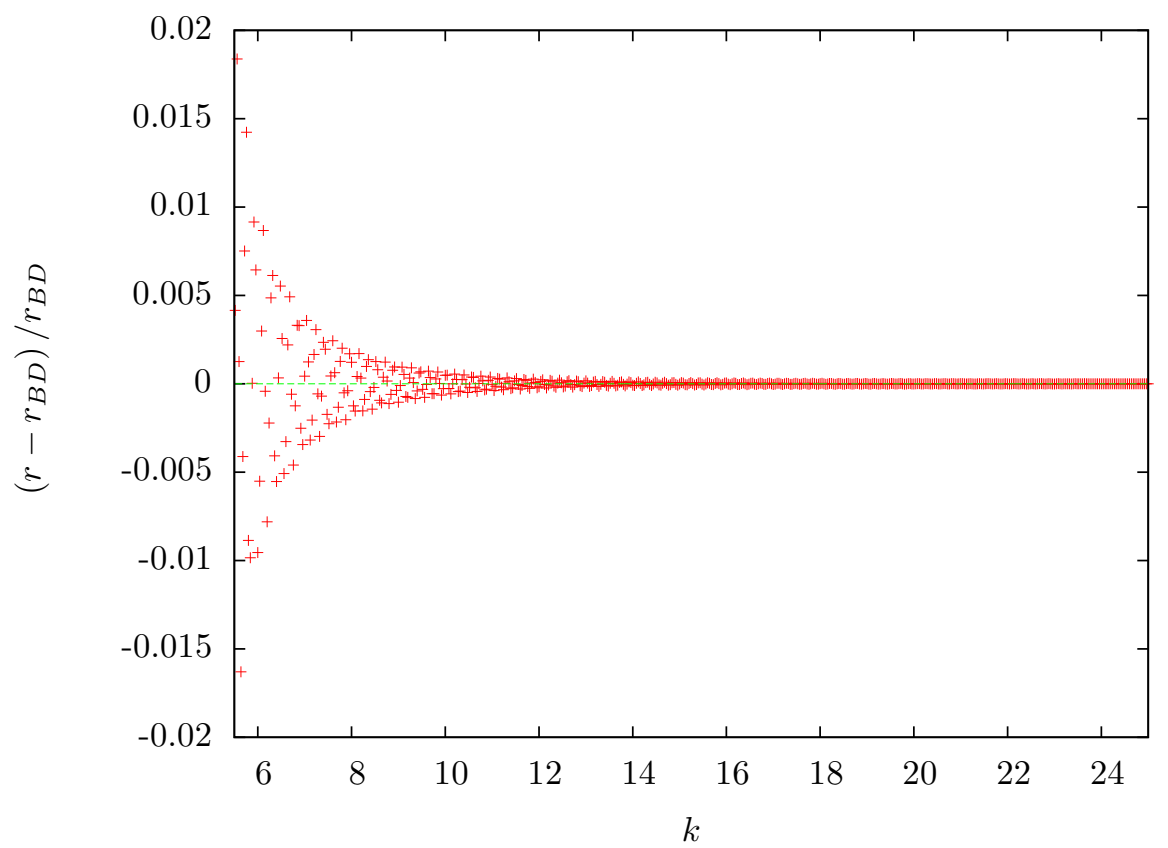

FIG. 8: Plot of the fractional difference between the LQC tensor to scalar ratio, $r_{\mathrm{LQC}}$ and the usual prediction from standard inflation, $r_{\mathrm{BD}}$ i.e. $\left(r_{\mathrm{LQC}}-r_{\mathrm{BD}}\right) / r_{\mathrm{BD}}$. The line is the average of points (see discussion below Eq. (5.15)). 


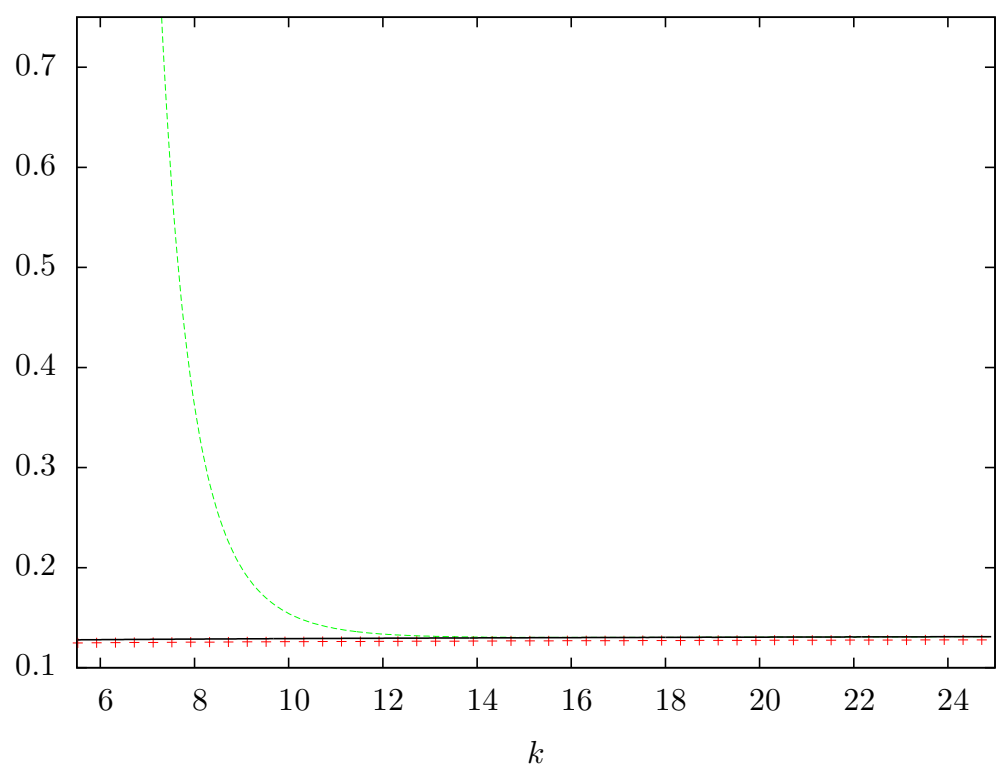

FIG. 9: Modification of the standard inflationary consistency relation $r_{\mathrm{BD}} \approx-8 n_{t}^{\mathrm{BD}}$, due to pre-inflationary dynamics. The green dashed line is $-8 n_{t}$, the solid black line is $-8\left(n_{t}-\right.$ $\left(d \ln \left(\left|\alpha_{k}\right|^{2}+\left|\beta_{k}\right|^{2}\right) / d \ln k\right)$ and the red crosses are the numerically calculated tensor to scalar ratio.

where, as before, we have averaged over the rapid oscillations.

Fig. 9 shows that this relation is satisfied to an excellent approximation in numerical simulations. It shows the imprint left by the pre-inflationary dynamics which is potentially observable: a deviations from the standard inflationary prediction at low $k$ 's.

\section{E. Checks of the numerics}

The accuracy of the simulations has been checked by carrying out multiple tests. We verified that:

- The norm of the evolved modes $e_{k}(\eta)$ and $q_{k}(\eta)$ is preserved under the numerical evolution.

- The Bogoluibov coefficients computed using Eq. (5.14) are time independent even though individual terms on the right side of this equation depend on time.

- The relation $\left|\alpha_{k}\right|^{2}-\left|\beta_{k}\right|^{2}=1$ is satisfied at all times and for all $k$.

- The two expressions for the power spectrum, Eq. (5.13) and Eq. (5.9) agree.

Although at the theoretical level some of these are just identities, they are excellent tests for the numerics.

As an example of convergence with respect to numerical precision, in Fig. 10 we have plotted the normalization condition, $\left|\alpha_{k}\right|-\left|\beta_{k}\right|-1$ calculated using three increasing levels of numerical precision (the step size of the internal integrator being reduced until the relative change at that step is less than one part in $10^{10}, 10^{15}$ and $10^{20}$ respectively). As can clearly be seen, we have already converged to better than one part in $10^{6}$ using the middle precision. 

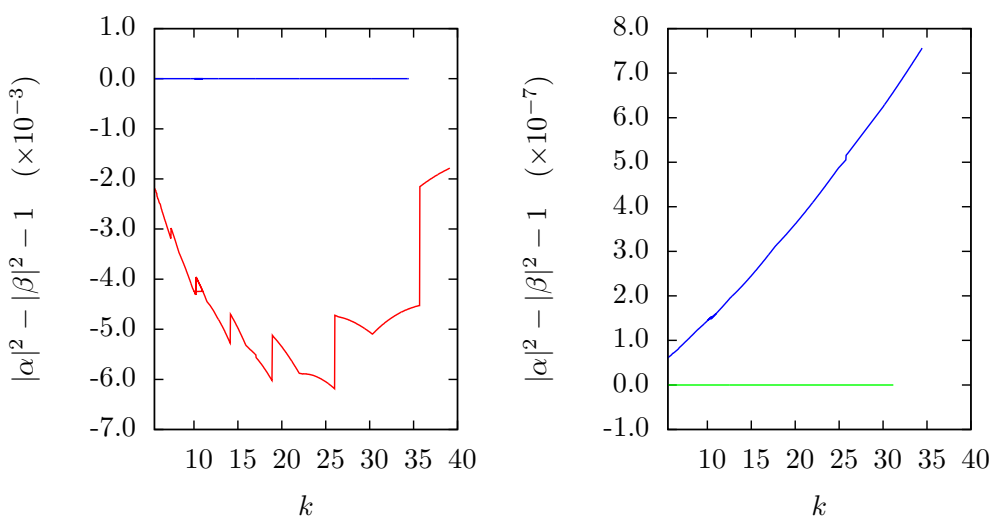

FIG. 10: These plots show the accuracy to which the normalization of the modes is maintained throughout the numerical integration. The Bogoluibov coefficients given in Eq. (5.8) satisfy $|\alpha|^{2}-$ $|\beta|^{2}=1$. These plots correspond to a (cosmic) time $\approx 5 \times 10^{4} t_{\mathrm{Pl}}$, for $\phi_{\mathrm{B}}=1.15$. The left plot shows the normalization for precision/accuracy goals within the numerical integrator being $10^{-10}$ (lower jagged line) and $10^{-15}$ (upper flat line), whilst the right plot is for precision/accuracy goals of $10^{-15}$ (upper, rising line) and $10^{-20}$ (lower flat line). Note the different scales on the two plots.

Unless otherwise stated, all plots have been calculated with the middle precision level, with convergence tested using the higher precision. Note that there is a (relative) loss of precision at large $k$, which becomes important, for example, in Fig. 6 and also in the calculation of the renormalized energy density at late times.

Another important check of the numerical accuracy comes from the fact that the $\alpha_{k}$ and $\beta_{k}$ coefficients given in Eq. (5.8) are time independent. An example of the level to which our numerical integration maintains this is given in Fig. 11. Here the normalization condition is plotted (as a function of $k$ ) for various times during the evolution (with $\phi_{B}=1.15$ ). A second (related) test comes from the time independence of the ratio $P_{\mathcal{R}}^{\Omega} / P_{\mathrm{BD}}$, which is plotted in Fig. 12. In both cases one again sees the loss of precision for large $k$. However, for the range of $k$ we are interested in, both quantities are conserved in time to better than one part in $10^{7}$.

\section{F. Summary}

In this section we have analyzed the effects of the pre-inflationary LQC space-time on the propagation of tensor and scalar perturbations. The numerical results shown in Fig. 5 support the physical picture presented in section IV B. Pre-inflationary geometry provided by LQC has a significant effect the evolution of modes with low $k$, which have wavelengths of the same order or larger than the curvature scale $k_{\mathrm{LQC}}$ at the bounce. However, the dynamics of modes with $k \gg k_{\mathrm{LQC}}$ is largely insensitive to the background geometry; they essentially evolve as if they were in flat space-time.

The relevant questions is then: What is the range of co-moving $k$ corresponding to $o b$ servable modes? As discussed in section IV B, this window depends on the value $\phi_{B}$ of the background inflaton at the bounce. The window is given by $k_{\min }=k_{o} \approx k_{\star} / 8.58$ and $k_{\max } \approx 2000 k_{\min }$ and, $k_{\star}$ increases with $\phi_{B}$ (see Table 1 ). If $\phi_{B} \gtrsim 1.2$ we have $k_{\min } \gtrsim k_{\mathrm{LQC}}$, and the evolved state is indistinguishable from the BD vacuum at the onset of inflation 


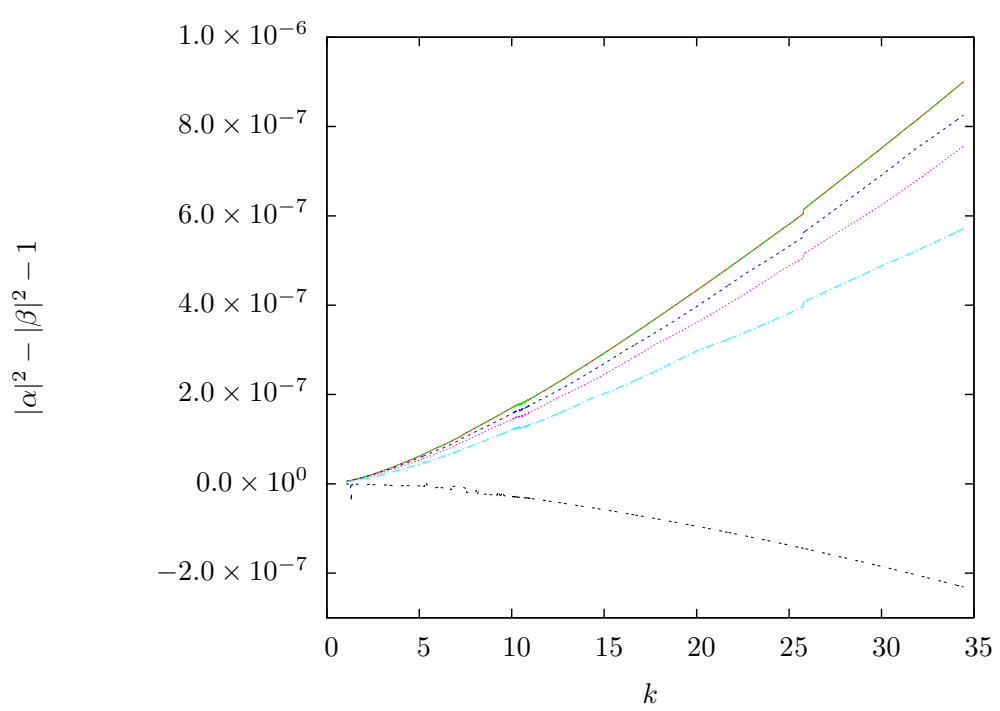

FIG. 11: Plot of the normalization condition of the Bogoluibov coefficients given in Eq. (5.8). In Fig. 10, we showed how this normalization converges with respect to the precision of the numerical integrator and here we demonstrate that this accuracy is maintained throughout the evolution. The various lines correspond to different time slices (from top to bottom (cosmic) time $=79,7.9 \times$ $10^{2}, 2.5 \times 10^{4}, 5.0 \times 10^{4}, 10^{6}, 3.9 \times 10^{6} t_{\mathrm{Pl}}$, respectively) for $\phi_{\mathrm{B}}=1.15$, using precision/ accuracy goals of $10^{-15}$.

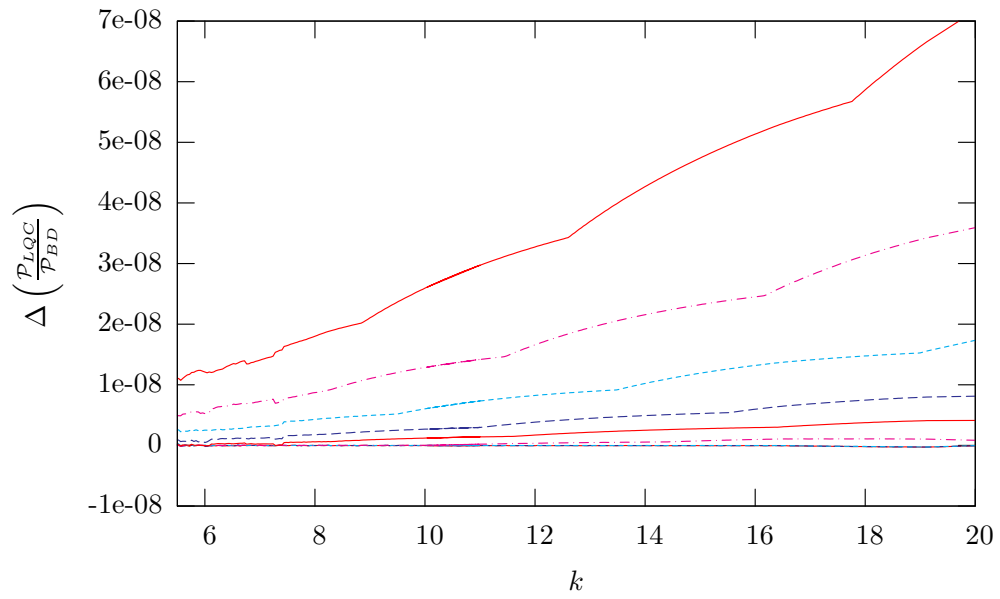

FIG. 12: The ratio of the LQC power-spectrum to the BD one for scalar perturbations is a constant in time, since it can be expressed entirely in terms of the Bogoluibov coefficients, $P_{\mathrm{LQC}} / P_{\mathrm{BD}}(k)=\left|\alpha_{k}+\beta_{k}\right|^{2}$. As a check of our numerics, this ratio was calculated at each out-put time. Here we have plotted the fractional change in this ratio (relative to that calculated at (cosmic) time $\stackrel{i}{t} \approx 1580 t_{\mathrm{Pl}}$ ) for a range of (cosmic) times (from bottom to top) $t=177,354,794,3550,6310,12600,25100,50100,10^{6}$ Planck seconds. $\Delta\left(P_{L Q C} / P_{B D}\right) \equiv$ $\left(P_{\mathrm{LQC}} / P_{\mathrm{BD}}(\tilde{t})-P_{L Q C} / P_{B D}(\hat{t})\right)\left(P_{B D} / P_{L Q C}\left(\hat{t}^{\circ}\right)\right)$. The growing deviations at large $k$ is an issue to do with numerical precision (see also Fig. 10 which shows the normalization of the modes). 
for observable modes. Therefore, for $\phi_{B} \gtrsim 1.2$ the present analysis provides a justification for the assumption of the Bunch-Davies vacuum at the onset of inflation for both tensor and scalar modes (at least for the observable range of $k$ ). It is important to emphasize that this conclusion is non-trivial because for those values of $\phi_{B}$ observable modes acquire trans-Planckian frequencies near the bounce and the standard treatment of cosmological perturbation in classical space-time is not applicable. The introduction of a quantum space-time was essential to incorporate those modes in a controlled fashion and to explicitly describe their evolution starting from the deep Planck regime.

Are there then any deviations from the standard predictions at all? The answer is in the affirmative for the small window in the parameter space given by $\phi_{B} \lesssim 1.2$. In this case, the state at the onset of inflation differs significantly from the BD vacuum, and the results for the spectrum of scalar and tensor perturbations differ from the standard predictions. For values of $\phi_{B}$ not too far from 1.2, the modes for which the power spectrum deviates from standard predictions have wave numbers smaller than $k_{\star}$. For these modes the WMAP observational error bars are large. For instance, for $\phi_{B}=1.15$ we have $k_{\min } \approx 1.07$ and modes for which deviation from standard predictions appear correspond to $\ell \lesssim 30$ in the WMAP angular decomposition. As a result, the LQC predictions are compatible with current data for the power spectrum. However, the fact that the state differs from the $\mathrm{BD}$ vacuum for those modes leads to a deviation from the standard 'consistency relation', $r_{\mathrm{BD}} \approx-8 n_{t}^{\mathrm{BD}}$, for low $k$ that may be seen in future observations (see Fig. 9). In addition, as pointed out in [10-12], the deviation from the BD vacuum at the onset of inflation can serve as a source of non-Gaussianity generated during inflation. Now, the presence of scalar $\mathrm{BD}$ quanta at the onset of inflation has been shown to lead to concrete effects in the CMB $[15,17]$, and in the distribution of galaxies [15, 16] which could be observed in the near future. If they are seen, these observations will significantly constrain the initial state for scalar perturbations at the onset of inflation. One would be able to make specific predictions for non-Gaussianities originating in LQC, thereby opening a novel avenue to observationally probe the Planck regime. Because the window, $\phi_{B} \lesssim 1.2$, in the parameter space for which new predictions arise is narrow, should these effects be observed, they would essentially pin down initial conditions for the background at the bounce, making more directed analysis and more detailed prediction feasible.

All these predictions were made under the assumption that our initial 'truncation approximation' is valid; i.e., that the back reaction of the perturbations on the (quantum) background is negligible. The goal of the next section is to analyze if this is the case.

\section{SELF-CONSISTENCY}

We began with the standard truncation of general relativity (coupled to a scalar field) that is used in the inflationary scenario and developed a quantum theory based on this truncation. A key question is whether this theory admits solutions which are consistent with the truncation approximation, i.e. in which the back reaction of perturbations can be neglected all the way from the bounce to the onset of the slow roll. Of course our conditions at the bounce on permissible states $\psi$ of perturbations are such that the approximation is satisfied initially. However, there is no a priori guarantee that it would continue to be satisfied during the entire evolution especially since it covers the 11 orders of magnitude in density and curvature.

In the first part of this section, we will report on the results obtained by numerical 


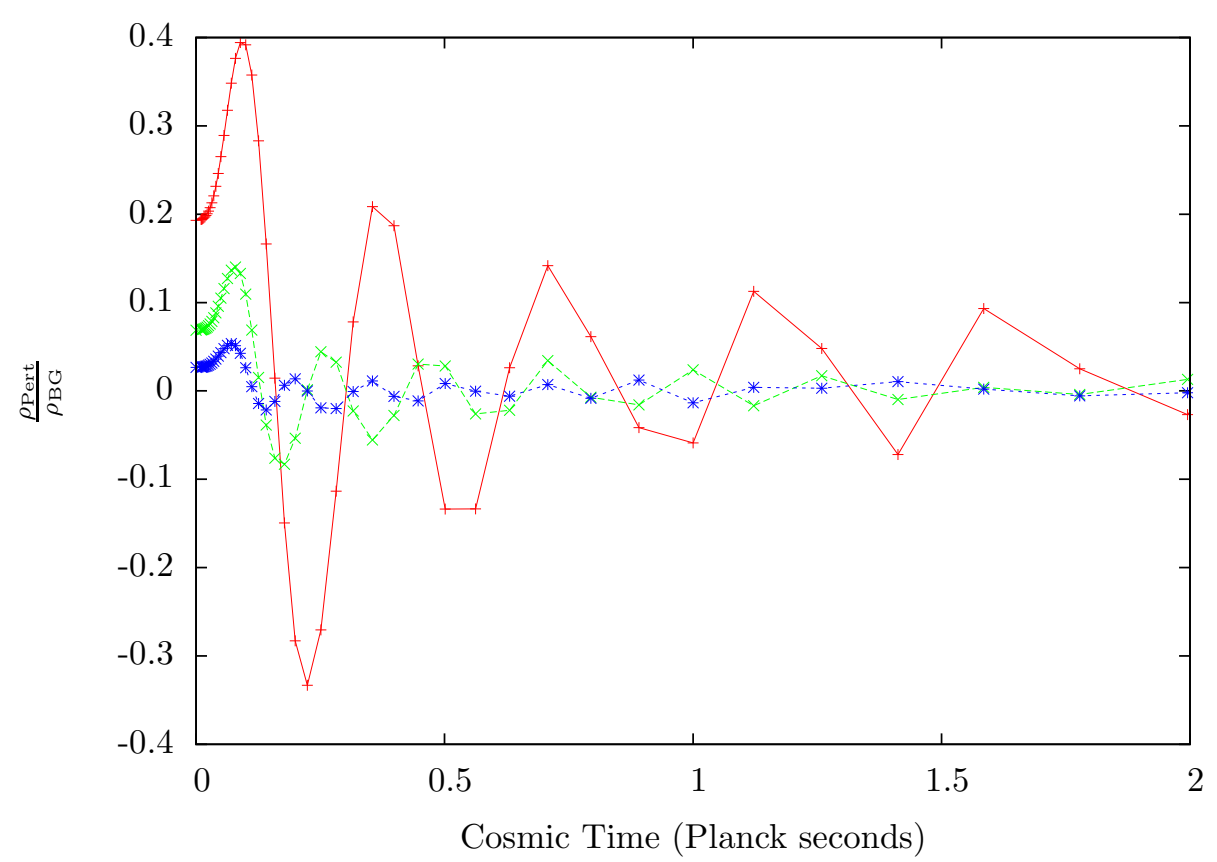

FIG. 13: Plot of the ratio of the energy density in the perturbations to that of the background for three values of $\phi_{\mathrm{B}}$. Here the $($ red $)+$ plot refers to $\phi_{\mathrm{B}}=1.21$; the $($ green $) \times$ to $\phi_{\mathrm{B}}=1.22$; and the (blue) $\star$ to $\phi_{\mathrm{B}}=1.23$ ). In order for the truncation scheme to be valid, we need to ensure $\rho_{\text {Pert }} / \rho_{\mathrm{BG}} \ll 1$, which is the case for $\phi_{\mathrm{B}} \geq 1.23$. This ratio remains small not only close to the bounce, but throughout the evolution, as shown in Fig. 14.

simulations using a specific quantum state of perturbations, $|\psi\rangle=\left|0^{\text {obv }}\right\rangle$. In the second part, we will use analytic considerations to argue that, if there is a state $\psi^{0}$ for which the back reaction is negligible, then there are infinitely many states 'close to' $\psi^{0}$ with this property.

Our focus will be on scalar perturbations; the situation with tensor perturbations is completely analogous. So far, our numerical methods allow us only to put upper bounds on the energy density in perturbations. But fortunately this is already sufficient to establish the existence of a large class of consistent solutions.

\section{A. Numerical analysis}

Conceptual aspects of the back-reaction issue were discussed in section VI.D of [19]. It was shown that a necessary and sufficient condition for the back reaction of perturbations $\psi$ on the background $\Psi_{o}$ to be negligible is that the expectation value $\langle\psi|\hat{\rho}(\vec{x}, \tilde{t})| \psi\rangle_{\text {ren }}$ should remain negligible compared to the energy density $\rho_{\mathrm{BG}}$ all the way from the bounce to the onset of slow roll.

Thus our task is to numerically evaluate the expression (2.29) of the renormalized energy density

$$
\left\langle 0^{\text {obv }}\left|\hat{\rho}^{(\mathcal{Q})}(\vec{x}, \tilde{t})\right| 0^{\text {obv }}\right\rangle_{\text {ren }}=\frac{\hbar}{2} \int_{k_{\min }}^{\infty} \frac{d^{3} k}{(2 \pi)^{3}}\left[\left|\dot{q}_{k}\right|^{2}+\frac{\left(\tilde{\mathfrak{A}}+k^{2}\right)}{\tilde{a}^{2}}\left|q_{k}\right|^{2}-\frac{1}{\tilde{a}^{4}} C^{(\mathcal{Q})}(k, \tilde{t})\right],
$$




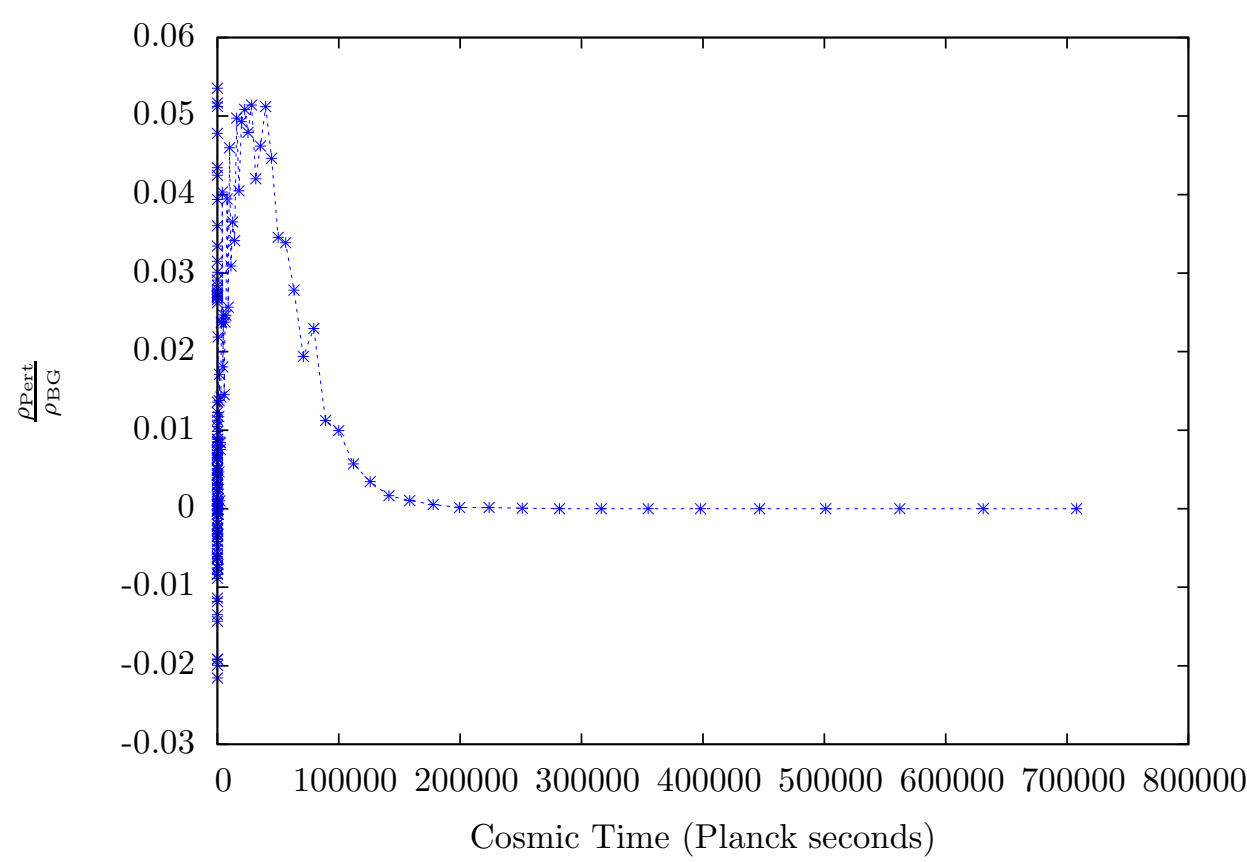

FIG. 14: Time dependence of the ratio of the energy density in the perturbations to that of the background (for $\phi_{B}=1.23$ ) over the entire evolution from the bounce (at $t_{B}=0$ ) to the onset of slow-roll inflation. The noisy peak at (cosmic) time $\approx 5 \times 10^{4}$ Planck seconds is due to numerical issues and in this region one should consider these points as an upper bound on the ratio.

where, the subtraction-term is given by $(2.30)$ :

$$
C^{(\mathcal{Q})}(k, \tilde{t})=k+\frac{\tilde{a}^{2} \dot{\tilde{a}}^{2}+\tilde{a}^{2} \tilde{\mathfrak{A}}}{2 \tilde{a}^{2} k}-\frac{\tilde{\mathfrak{A}}^{2}+2 \tilde{\mathfrak{A}} \dot{\tilde{a}}^{2}-3 \dot{\tilde{a}^{4}}+2 \tilde{a} \dot{\tilde{a}}^{2} \ddot{\tilde{a}}-\tilde{a}^{2} \ddot{\tilde{a}}^{2}+2 \tilde{a} \dot{\tilde{a}}(-\dot{\tilde{\mathfrak{A}}}+\tilde{a} \ddot{\tilde{a}})}{8 k^{3}} .
$$

Recall that the infrared cut-off $k_{\min }$ is given by $k_{\min }=k_{\star} / 8.58$. Since the value of $k_{\star}$ is determined by $\phi_{\mathrm{B}}$, the co-moving infrared cut-off is simply a fixed number for any given state $\Psi_{o}$ of the background geometry. What would happen if one were to choose a lower $k_{\min }$ as the infra-red cut-off? In this case, the results discussed below will change only in the value $\phi_{\mathrm{B}}$ above which we have self-consistent solutions of the truncated theory. Furthermore, the exponential relation between $\phi_{\mathrm{B}}$ and $k_{\star}$ shown in Fig. 3, implies that the required change in $\phi_{B}$ will only be logarithmic in the change in $k_{\min }$.

Since $k_{\min }$ increases with $\phi_{\mathrm{B}}$, one might expect that an increase in $\phi_{B}$ will result in a decrease in the expectation value of the energy density at any given particular time, simply because of the decrease in the domain of integration. Our numerics bear this out. The results are plotted in Fig. 13 for early times near the bounce and in Fig. 14 for all times from the bounce to the onset of slow roll. These results show, in particular, that for $\phi_{B} \geq 1.23$ we have,

$$
\frac{\langle\psi|\hat{\rho}| \psi\rangle_{\mathrm{ren}}(\tilde{t})}{\rho_{\mathrm{BG}}(\tilde{t})}<0.05 \quad \forall \tilde{t} .
$$

Thus the test field approximation holds if $\phi_{\mathrm{B}} \geq 1.23$. It may hold also for lower values of $\phi_{\mathrm{B}}$ because, as indicated in Figs. 14 and 15 and discussed below, what we control numerically is an upper bound on the energy density in perturbations. 


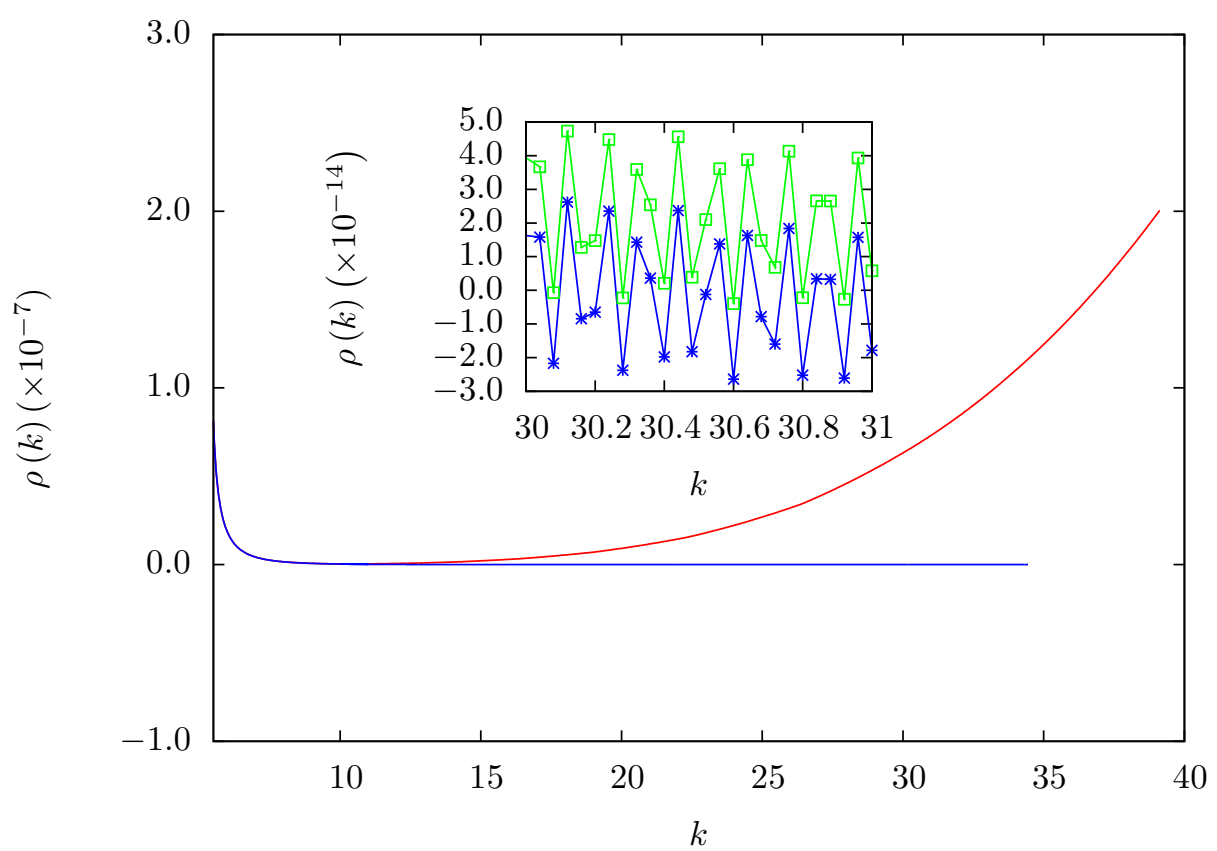

FIG. 15: An illustration of the importance of numerical precision for high $k$. The plot shows the integrand $\rho(k)$ of the energy density in perturbations for $\phi_{B}=1.15$ and at time $t \approx 5 \times 10^{4} t_{\mathrm{Pl}}$. The corresponding background energy density $\rho_{\mathrm{BG}}$ is only $\approx 10^{-9}$ ! The upper (red) solid line was evaluated with the lowest precision/accuracy goal $10^{-10}$, whilst the lower (blue) line corresponds to precision accuracy of $10^{-25}$. The inset (where the y-axis is magnified by another factor of $10^{7}$ ) shows that there are delicate cancelations between positive and negative values of $\rho(k)$. In the inset the upper (green) plot corresponds to precision/ accuracy goal of $10^{-20}$ while the lower (blue) to $10^{-25}$.

Numerically these calculations are challenging because the integrand rapidly builds up a fast (but decaying) oscillation in $k$ (similar to Fig. 4). In addition, for large $k$, even a very small loss of precision can result in a significant error, since the renormalization procedure subtracts two diverging terms. This problem is exacerbated by the fact that there are times when even extremely small numerical errors (of the order of one part in $10^{15}$ ) can be comparable to the background energy density; see Fig. 15. But, typically, this is not an issue for the early phase where quantum gravity dominates and the background energy density is high, or the very late time evolution where the energy density in the perturbations is suppressed by the large scale factor. The problem is serious only for the intermediate times, $\tilde{t} \approx 50,000 t_{\mathrm{Pl}}$, when the calculations of the expectation value of the energy density are dominated by numerical noise. However we have used sufficient precision to ensure that this noise is always less than half a percent of the background energy density. This allows us to ensure that Eq. (6.3) is satisfied. But because of this, our numerical estimates should be considered as upper bounds. 


\section{B. Analytical considerations}

In section VIA we used numerics to provide an explicit example of a state for which our underlying truncation scheme is self-consistent. However, one would also like to know whether this particular example is special or if there are 'many' other states in the Hilbert space $\mathcal{H}_{1}$ for which our truncation is also self-consistent. Of course, such questions have to be phrased with care because $\mathcal{H}_{1}$ is infinite dimensional. In this section we will provide an analytical argument to establish the following result: If a state $|\psi\rangle$ in $\mathcal{H}_{1}$ is such that $\langle\psi|\hat{\rho}| \psi\rangle_{\text {ren }}$ is very small compared to the energy density $\rho_{\mathrm{BG}}$ in the background, then the same is true for every state $|\tilde{\psi}\rangle$ in an open neighborhood of $\psi$ in $\mathcal{H}_{1}$. Together with the numerical example $|\psi\rangle=\left|0^{\text {obv }}\right\rangle$ given in the previous subsection, this result establishes that there are 'many' states in $\mathcal{H}_{1}$ for which the truncation approximation is valid. Since our considerations are all analytic, in this subsection we will work with the conformal time $\tilde{\eta}$ of the dressed effective metric $\tilde{g}_{a b}$ (rather than the cosmic time $\tilde{t}$ ).

Let $|\psi\rangle$ then be a 4 th adiabatic order vacuum in $\mathcal{H}_{1}$ such that,

$$
\langle\psi|\hat{\rho}| \psi\rangle_{\text {ren }}(\tilde{\eta}) \ll \rho_{\text {BG }}(\tilde{\eta}) \quad \forall \tilde{\eta} \in\left(\tilde{\eta}_{\text {ini }}, \tilde{\eta}_{\text {final }}\right),
$$

where $\left(\tilde{\eta}_{\text {ini }}, \tilde{\eta}_{\text {final }}\right)$ is the dynamical range we are interested in. Now consider a second 4 th adiabatic order vacuum $|\tilde{\psi}\rangle$ in $\mathcal{H}_{1}$. Let $q_{k}$ and $\tilde{q}_{k}$ be any 4 th order bases that define $|\psi\rangle$ and $|\tilde{\psi}\rangle$ and let the Bogoluibov transformation relating them be given by

$$
\tilde{q}_{k}=\alpha_{k} q_{k}+\beta_{k} q_{k}^{\star} .
$$

One can easily check that the difference of the expectation values of the renormalized energy density with respect to $|\tilde{\psi}\rangle$ and $|\psi\rangle$ is given,

$$
\begin{aligned}
\Delta \rho[\tilde{\psi}, \psi] & \equiv\langle\tilde{\psi}|\hat{\rho}| \tilde{\psi}\rangle-\langle\psi|\hat{\rho}| \psi\rangle \\
& =\frac{\hbar}{(2 \pi)^{3} \tilde{a}^{2}} \int d^{3} k\left[\left|\beta_{k}\right|^{2}\left(\left|q_{k}^{\prime}\right|^{2}+\left(k^{2}+\tilde{\mathfrak{A}}\right)\left|q_{k}\right|^{2}\right)+\operatorname{Re}\left[\alpha_{k} \beta_{k}^{\star}\left(q_{k}^{\prime 2}+\left(k^{2}+\tilde{\mathfrak{A}}\right) q_{k}^{2}\right)\right]\right]
\end{aligned}
$$

where we have used the relation $\left|\alpha_{k}\right|^{2}-\left|\beta_{k}\right|^{2}=1$. From the previous equation, by taking into account that $-|z| \leq \operatorname{Re}(z) \leq|z|$ for any complex number $z$, we can obtain the following bound for $\Delta \rho$

$$
\frac{\hbar}{(2 \pi)^{3} \tilde{a}^{2}} \int d^{3} k B_{-}\left(\left|q_{k}^{\prime}\right|^{2}+\left(k^{2}+\tilde{\mathfrak{A}}\right)\left|q_{k}\right|^{2}\right) \leq \Delta \rho \leq \frac{\hbar}{(2 \pi)^{3} \tilde{a}^{2}} \int d^{3} k B_{+}\left(\left|q_{k}^{\prime}\right|^{2}+\left(k^{2}+\tilde{\mathfrak{A}}\right)\left|q_{k}\right|^{2}\right),
$$

where $B_{ \pm}=\left|\beta_{k}\right|^{2}\left(1 \pm \sqrt{1 /\left|\beta_{k}\right|^{2}+1}\right)$. Finally, since $|\psi\rangle$ and $|\tilde{\psi}\rangle$ are both $4^{\text {th }}$ adiabatic order states, the $\beta_{k}$ coefficients decay sufficiently fast with $k$ so that the integrals in these bounds are convergent. Therefore, the bounds are finite. Furthermore, all time dependent quantities are continuous in $\tilde{\eta}$ whence the difference $\Delta \rho[\tilde{\psi}, \psi](\tilde{\eta})$ has an upper bound in the closed time interval under consideration. Since this bounds scales with $B_{k}^{ \pm}$, we can make $\sup \Delta \rho[\tilde{\psi}, \psi](\tilde{\eta})$ arbitrarily small simply by rescaling the $\beta_{k}$ by a sufficiently small constant. Thus we have an (open) neighborhood of the state $|\psi\rangle$, parameterized (for each mode) by 
$\left|\beta_{k}\right|$, in which the change in the expectation value of the energy density in the perturbations is also very small compared to the background for all times between the bounce and the onset of the slow roll.

To summarize, in this section we have shown that if the state $\Psi_{o}$ of the background geometry is chosen so that $\phi_{\mathrm{B}} \geq 1.23$, then $\left|\Psi_{o}\right\rangle \otimes\left|0^{\text {obv }}\right\rangle$ provides a self-consistent solution to our truncated quantum theory. Furthermore, there are infinitely many states $|\tilde{\psi}\rangle$ 'close to' $\left|0^{\text {obv }}\right\rangle$ for which $\left|\Psi_{o}\right\rangle \otimes|\tilde{\psi}\rangle$ shares this property. Each of these states provides an admissible extension of the standard inflationary scenario to the Planck regime. Finally, so far our numerical techniques have provided only upper bounds for the renormalized energy density. It is conceivable that future work will show that the self consistent solutions exist also for lower values of $\phi_{\mathrm{B}}$.

\section{SUMMARY AND DISCUSSION}

In the standard inflationary scenario one works with quantum fields representing first order perturbations on a FLRW solution to Einstein's equations. One assumes that these quantum fields are in the BD vacuum at the onset of slow roll, works out their evolution and calculates the power spectra and other correlation functions at the end of inflation. The fact that one starts the calculations at the onset of slow roll is often justified on the basis that pre-inflationary dynamics will not affect any of the observable predictions. This belief stems from the argument that modes seen in the CMB cannot be excited during the pre-inflationary dynamics because, when evolved back in time starting from the onset of the slow roll, their physical wave lengths $\lambda_{\text {phy }}$ continue to remain within the Hubble radius $\mathfrak{R}_{\mathrm{H}}$ all the way to the big bang. However, this argument is flawed on two reasons. First, what matters to the dynamics of these modes is the curvature radius $\mathfrak{R}_{\text {curv }}=\sqrt{6 / R}$ determined by the Ricci scalar $R$, and not $\mathfrak{R}_{\mathrm{H}}$, and the two scales are equal only during slow roll. Thus we should compare $\lambda_{\text {phy }}$ with $\mathfrak{R}_{\text {curv }}$ in the pre-inflationary epochs. The second and more important point is that the pre-inflationary evolution should not be computed using general relativity, as is done in the argument given above. One has to use an appropriate quantum gravity theory since the two evolutions can well be very different in the Planck epoch. Then modes that are seen in the CMB could well have $\lambda_{\text {phy }} \gtrsim \mathfrak{R}_{\text {curv }}$ in the pre-inflationary phase. If this happens, these modes would be excited and the quantum state at the onset of the slow roll could be quite different from the BD vacuum. Indeed, the difference could well be so large that the resulting power spectrum is incompatible with the amplitude and the spectral index observed by WMAP. In this case, that particular quantum gravity scenario would be ruled out. On the other hand, the differences could be more subtle: the new power spectrum for scalar modes could be the same but there may be departures from the standard predictions that involve tensor modes or higher order correlation functions of scalar modes, changing the conclusions on non-Gaussianities. In this case, the quantum gravity theory would have interesting predictions for future observational missions. Thus, as we emphasized in section $\mathrm{IV}$, pre-inflationary dynamics can provide an avenue to confront quantum gravity theories with observations.

In this paper we investigated these possibilities in the context of loop quantum gravity. In the standard inflationary scenario, one starts by truncating general relativity, keeping only the FLRW solutions (coupled to a scalar field) and first order perturbations thereon. Therefore, in our analysis we began in section II with the truncated phase space $\Gamma_{\text {Trun }}=$ $\Gamma_{o} \times \tilde{\Gamma}_{1}$ where $\Gamma_{o}$ is the FLRW phase space of homogeneous, isotropic fields and $\tilde{\Gamma}_{1}$ is the phase 
space of gauge invariant first order perturbations. We then passed to the quantum theory of the entire $\Gamma_{\text {Trun }}$. Consequently in the resulting framework tensor and scalar perturbations $\hat{\mathcal{T}}$ and $\hat{\mathcal{Q}}$ propagate on a quantum FLRW geometry encapsulated in a wave function $\Psi_{o}$. This quantum geometry is non-singular; the big bang of general relativity is replaced by a quantum bounce. Therefore we can directly face the trans-Planckian issues. We found that the existence of trans-Planckian modes is not a problem in itself. The key question is whether these modes contribute so much to the energy density in the perturbations that the back reaction of perturbations on $\Psi_{o}$ cannot be neglected. This could easily happen especially in the Planck regime. If it did, our 'truncation' strategy would fail to be selfconsistent. Therefore the challenge is to develop techniques to calculate the energy density in perturbations all the way from the bounce to the onset of the slow roll and compare it with background energy density. Finally, as we saw in section II B 2, LQC dynamics reveals several features in the Planck regime that are very surprising from the perspective of general relativity which has shaped intuition in the standard inflationary scenario.

The underlying FLRW quantum geometry streamlines the analysis by providing the necessary control on calculations in the deep Planck regime. However, we are now faced with the challenge of investigating the dynamics of quantum fields $\hat{\mathcal{T}}, \hat{\mathcal{Q}}$ on quantum geometries. At first this problem seems formidable. But fortunately there is a key simplification [19, 48]: Mathematically this evolution is completely equivalent to that of $\hat{\mathcal{T}}, \hat{\mathcal{Q}}$ propagating on a dressed, effective metric $\tilde{g}_{a b}$, which incorporates the appropriate quantum properties of the state $\Psi_{o}{ }^{11}$ This occurs because the dynamics of test quantum fields does not experience all the details of the probability amplitude for various FLRW metrics encapsulated in $\Psi_{o}$; it is sensitive only to a few moments of this distribution. The phenomenon is analogous to the propagation of light in a medium where all the complicated interactions of the Maxwell field with the atoms in the medium can be captured just in a few parameters such as the refractive index. This unforeseen outcome enabled us to 'lift' the adiabatic regularization and renormalization techniques, normally used in QFT in classical FLRW space-times, to QFT on quantum FLRW geometries, thereby providing the necessary control on the dynamics of $\hat{\mathcal{T}}, \hat{\mathcal{Q}}$ on the quantum geometry $\Psi_{o}$.

In section III we specified a class of initial conditions at the bounce for the quantum state $\Psi_{o} \otimes \psi$ of the combined system, $\Psi_{o}$ describing the background and $\psi$ describing perturbations. The permissible states $\Psi_{o}$ are required to be sharply peaked on certain effective, bouncing trajectories in the phase space, which are well-understood. It turns out that, except for a tiny portion, $\phi_{\mathrm{B}}<0.93$, of the allowed range of values for the background inflaton at the bounce, all effective trajectories encounter the slow roll compatible with the 7 year WMAP data sometime in their future evolution [6]. This was an unanticipated result. However, it does not mean that the background states $\Psi_{o}$ are generic. In fact, they are very special because they are required to be sharply peaked. For the states $\psi$ of perturbations, we imposed three conditions: i) Regularity: They be of 4th adiabatic order so that the expectation value $\langle\psi|\hat{\rho}| \psi\rangle_{\text {ren }}$ of the renormalized energy density operator is well defined; ii) Symmetry: They be invariant under spatial translations and rotations, i.e., under sym-

11 While the standard inflationary dynamics of tensor modes $\hat{\mathcal{T}}$ is the same as that of a massless scalar field on a FLRW metric $g_{a b}$, that of the Mukhanov-Sasaki field $\hat{\mathcal{Q}}$ representing scalar perturbations involves also an 'external potential' $\mathfrak{A}$. For both modes, $g_{a b}$ is replaced by a quantum corrected metric $\tilde{g}_{a b}$ in LQC. For the scalar mode, in addition, $\mathfrak{A}$ is replaced by a quantum corrected $\tilde{\mathfrak{A}}$. 
metries of the background geometry; and, iii) Initial compatibility with truncation: At the bounce, $\langle\psi|\hat{\rho}| \psi\rangle_{\text {ren }}$ be negligible compared to the background energy density $\rho_{\mathrm{BG}}$. There is a large class of such states. We discussed in detail a specific example $|\psi\rangle=\left|0^{\text {obv }}\right\rangle$, called the 'obvious vacuum of the 4th adiabatic order', in section III, and mentioned a few other examples section V C.

In sections IV and $\mathrm{V}$ we used numerical methods to evolve these initial conditions and obtained the power spectra for scalar and tensor perturbations. The key free parameter in this analysis is the value $\phi_{\mathrm{B}}$ of the background inflaton at the LQC bounce because it determines the effective trajectory on which $\Psi_{o}$ is peaked. For $\psi$, we first carried out a few simulations using various choices discussed in section V C to ensure robustness of the final results, and then focused on the choice $|\psi\rangle=\left|0^{\text {obv }}\right\rangle$ because it has several attractive properties.

As explained in sections V and VI, several of the numerical simulations require very high precision (see, e.g., Fig. 15). Furthermore, it turns out that the integration time increases very rapidly with $\phi_{\mathrm{B}}$. Therefore, we had to restrict our simulations to $\phi_{\mathrm{B}} \lesssim 2$. Fortunately, as summarized below, the interval $\phi_{\mathrm{B}} \lesssim 2$ covers the physically most interesting range. Our numerical simulations brought out an unforeseen feature: The LQC power spectra are essentially insensitive to the precise value of $\phi_{B}$. However, in the CMB we have access only to a finite range of wave numbers. The 7 year WMAP data in particular covers a range $\sim\left(k_{o}, 2000 k_{o}\right)$ where $k_{o}$ is the co-moving wave number of the mode whose physical wave length equals the radius $\mathfrak{R}_{\mathrm{LS}}$ of the observable universe at the surface of last scattering. In $\mathrm{LQC}$, the value of $k_{o}$ is dictated by $\phi_{\mathrm{B}}$ (in our scale factor convention $a_{\mathrm{B}}=1$ ). Therefore, in our analysis the window of modes (in the $k$ space) that can be seen in the CMB is dictated by the value of $\phi_{\mathrm{B}}$, and moves rapidly to the right along the $k$ axis as $\phi_{\mathrm{B}}$ increases.

Are there values of $\phi_{\mathrm{B}}$ then, for which LQC predicts deviations from the standard inflationary scenario for modes in the observable window? As mentioned in the beginning of this section, on rather general grounds we expect that a mode would be excited in the preinflationary dynamics if $\lambda_{\text {phy }} \gtrsim \mathfrak{R}_{\text {cuv }}$ at any time. A detailed analysis of the pre-inflationary dynamics of the LQC background geometry shows that such modes can be seen in the CMB only if $\phi_{B}<1.2$ (see section IV B). In this case, for observable modes, the state $|\psi\rangle$ has nontrivial excitations over the $\mathrm{BD}$ vacuum at the onset of inflation. If $\phi_{\mathrm{B}}=1.15$ for example, the reference mode used in WMAP corresponds to $k_{\star}=9.17$ in our conventions and as Fig. 5 shows, the LQC predictions for the power spectrum and the spectral index at $k=9.17$ are indistinguishable from those of standard inflation. Thus, the prediction is observationally viable. However, for $k<9.17$ - which correspond to $\ell \lesssim 30$ in the angular decomposition used by WMAP - there are significant differences between LQC and the standard inflation because of the excitations over the $\mathrm{BD}$ vacuum. Because the observational error bars for low $\ell$ are large, both predictions are observationally viable. Furthermore, since the LQC excitations over the BD vacuum are the same for scalar and tensor modes, the ratio $r$ of the tensor to scalar power spectra is unchanged from that in standard inflation. However, the standard 'consistency relation' $r_{\mathrm{BD}}=-8 n_{t}^{\mathrm{BD}}$ is modified. Future measurements of tensor modes should be able to distinguish between the two. This departure from the BD vacuum also has implications for the CMB and galaxy distribution [11-14] and observational tests for such effects have already been proposed [15-17]. Thus, there are differences between the LQC and the standard inflationary predictions if $\phi_{\mathrm{B}}<1.2$.

What if $\phi_{\mathrm{B}}>1.2$ ? Then we would have $\lambda_{\text {phy }} \ll \mathfrak{R}_{\text {cuv }}$ throughout the pre-inflationary evolution for all the modes that can be seen in the CMB. Therefore, the physical reasoning 
strongly suggests that they would not 'experience' significant curvature and would be in the $\mathrm{BD}$ vacuum at the onset of inflation. In this case, pre-inflationary dynamics would not modify standard inflationary predictions for observable modes. Numerical simulations made this semi-quantitative considerations precise in that our plots provide sharp figures for excitations over the BD vacuum for various values of $\phi_{\mathrm{B}}$. In particular, if $\phi_{\mathrm{B}}>1.4$, we find that the excitations are so small that the departures predicted by LQC would be negligible for any of the forthcoming observation missions. This also implies that the fact that we had to limit our numerical simulations to $\phi_{\mathrm{B}} \lesssim 2$ is not physically restrictive.

To summarize, by analyzing the pre-inflationary dynamics in detail we arrived at two main conclusions. First, there do exist natural initial conditions at the bounce which lead to a completion of the standard inflationary scenario to include the quantum gravity regime. In this completed theory, one has a consistent evolution all the way from the deep Planck regime that accounts for the inhomogeneities seen in the CMB. Since the origin of the large scale structure can be traced back to these inhomogeneities, now one can systematically trace back the seeds of this structure to the quantum fluctuations of the initial state at the $L Q C$ bounce itself. Second, there is a narrow window in the $\phi_{\mathrm{B}}$ parameter space for which the state at the onset of inflation would not be the BD vacuum. While the LQC and the standard inflation predictions are both compatible with current observations, future observations should be able to distinguish between the two. Thus, there is a potential to extend the reach of observational cosmology all the way to the Planck scale. Of course, since the window is narrow, the 'a priori' probability of its being realized in Nature is small. This is compensated by the fact that, if observations are compatible with $\phi_{\mathrm{B}}$ being in this window, the initial conditions would be narrowed down tremendously, making very detailed calculations and predictions feasible.

Finally, in section VI we investigated the issue of whether our truncation strategy is self-consistent, i.e., whether in the solutions $\Psi_{o} \otimes \psi$ we analyzed the energy density in the perturbations can be neglected compared to that in the background all the way from the bounce to the onset of inflation. This is a difficult issue on two accounts and to our knowledge it had not been analyzed in the Planck regime in any approach. The first difficulty is conceptual: one needs a systematic framework to compute the renormalized energy density. As discussed above, we were able to construct this framework using the well-developed adiabatic renormalization theory on cosmological space-times because of the exact mathematical equivalence between QFT on the LQC quantum geometries $\Psi_{o}$ and QFT on dressed effective metrics $\tilde{g}_{a b}$ determined by $\Psi_{o}$. The second set of difficulties comes from numerics: because of rapid oscillations of the integrand of $\langle\psi|\hat{\rho}| \psi\rangle_{\text {ren }}$ in the $k$ space, and because the background energy density itself diminishes rapidly as one evolves to the future of the bounce, one requires very high accuracy and numerical precision. We were not able to calculate $\langle\psi|\hat{\rho}| \psi\rangle_{\text {ren }}$ over the entire evolution from the bounce to the onset of inflation to the desired precision. However, we were able to provide an upper bound on this quantity. For $\phi_{B} \geq 1.23$, these upper bounds suffice to guarantee that the back reaction of perturbations can indeed be ignored for the states $\Psi_{o} \otimes \psi$ we considered. This suffices to show that there is a satisfactory extension of the standard inflationary scenario in which it is consistent to ignore the back reaction also in the pre-inflationary epoch. However, for lower values of $\phi_{\mathrm{B}}$ - particularly those for which the state at the onset of inflation is significantly different from the BD vacuum - we have not been able to demonstrate that the truncation scheme is self consistent. We hope to return to a detailed analysis of this issue in the near future. 
We will conclude with a few remarks.

- While this paper was focused on inflation, the underlying framework developed in [19] is much more general. Together, the two investigations offer some general lessons that could be useful also in other paradigms for the early universe. For example, we saw that, in the investigation of whether a mode would be dynamically excited, what matters is not the Hubble radius which often dominates this discussion but the curvature radius $\mathfrak{R}_{\text {curv }}$ determined by the scalar curvature of the background. As Fig. 1 illustrates, the evolution of $\mathfrak{R}_{\text {curv }}$ in the early universe can be quite involved and therefore non-intuitive, especially in the regime in which Einstein's equations receive significant corrections. Therefore, it is important to calculate this quantity throughout the period of interest. A second issue involves back reaction [79]. Since it is negligible during slow roll, the issue has often been ignored in the investigations of pre-inflationary dynamics as well as in discussions of alternatives to inflation. However, the issue is important especially in the Planck era and our numerical simulations showed that it is quite subtle. In particular, there is a correlation between the fact that modes with $\lambda_{\text {phy }} \gtrsim \mathfrak{R}_{\text {curv }}$ are excited during evolution and the observation from numerics that these modes make significant contribution to the energy density in perturbations. Now, if there is sufficient inflation, then the modes that are relevant for CMB observations tend to have very small $\lambda_{\text {phy }}$ in the Planck regime. On the other hand, if there is no inflation at all, it is not easy to achieve sufficient expansion between the bounce and the CMB epoch for these modes to have $\lambda_{\text {phy }} \ll \mathfrak{R}_{\text {curv }}$ in the Planck era. These general physical considerations suggest that in scenarios without inflation there is a danger that the back reaction may not be negligible especially in the Planck regime. Therefore it is important to check the consistency of first order perturbation theory in those cases.

- In recent years, there have been a number of interesting investigations of possible LQC corrections to inflationary dynamics (for a brief summary, see section II of [19].) Some of these have focused on pre-inflationary dynamics studied in this paper (see especially $[47,80])$. The distinguishing features of our analysis are: i) It is based on the general truncation strategy which has been successfully employed in LQG in other problems; ii) The issue of the initial state has been investigated in a stream-lined fashion; iii) Numerical simulations are better controlled and considerably more extensive. Furthermore, the physics behind the main findings is understood and discussed in detail; iv) Issues of regularization of composite operators and renormalization of energy density have been addressed for the first time. They enabled us to systematically check if the back reaction can be ignored.

- The truncation strategy used in this series of papers is motivated by two considerations: i) We begin with the phase space $\Gamma_{\text {Trun }}$ that underlies almost all investigations of the early universe; and, ii) The same truncation philosophy underlies other successful applications of LQG such as the calculation of the graviton propagator. Nonetheless, it would clearly be much better if we could start with a full quantum gravity theory and then descend to this truncation systematically. Could the final results be significantly different as far as observational issues are concerned? We believe that the answer is in the negative at least in cases where the truncation procedure can be shown to admit self-consistent solutions. For, in general relativity one routinely expects first order perturbations whose back reaction is negligible to provide an excellent approximations to the phenomenological predictions of the exact theory, and there is no obvious reason why the situation would be different in quantum gravity. As a simple example to illustrate our view of the effectiveness 
of the truncated theory, consider the Dirac solution of the hydrogen atom. Because it assumes spherical symmetry prior to quantization, this truncation excludes photons from the beginning. Therefore, at a conceptual level, it is very incomplete. Yet, as far as experiments are concerned, it provides excellent approximations to answers provided by full quantum electrodynamics till one comes to the Lamb shift. We expect the situation to be similar for our truncated theory: Conceptually it is surely quite incomplete vis a vis full LQG, but we expect the full theory to provide only small corrections to observable effects.

- However, even apart from the issue of full LQG, the current framework can be improved in a number of ways in the near future. We will complete our remarks by providing a few examples.

$\star$ Extensions: First, it would be desirable to extend the framework in several directions. Inclusion of a positive cosmological constant would be straightforward. While it would add a few conceptual twists along the lines of [31], the effect on pre-inflationary dynamics investigated here would be totally negligible because the phenomenological value of the cosmological constant is so small. Second, it would be worthwhile to extend the calculations to include multi-inflatons, especially since multi-inflatons have already been considered in LQC [81]. Are there any significant changes in the results on power spectra? A third extension would be to investigate cases where $\phi_{\mathrm{B}}$ is much larger than 2 . We gave simple physical arguments that for $\phi_{\mathrm{B}}>1.4$ the LQC results would be indistinguishable from those of standard inflation as far as foreseeable observations are concerned. But it is not impossible that these considerations neglected to take into account some subtle feature of the background dynamics for large $\phi_{\mathrm{B}}$. Only explicit calculations can decide. However, since numerical simulations become prohibitively expensive for large $\phi_{\mathrm{B}}$, a combination of analytical approximations and numerics will be needed. Finally, our preliminary results indicate that, if one were to extend the detailed numerical calculations all the way to $\phi_{\mathrm{B}}=$ 0.93 , the lowest value that is compatible with the 7 year WMAP data according to [6], one would find that the state at the onset of inflation is so different from the BD vacuum that one has to significantly revise the value of the inflaton mass used in this paper. We have made a first pass at redoing the calculation using a cyclic procedure described at the end of section VA and plan to carry out a detailed calculation. However, a more systematic and efficient procedure extracted from the standard routines used in the WMAP data analysis would be highly desirable.

* Quantum theory of perturbations: To make direct contact with calculations in the standard inflationary scenario, we used a Fock representation for quantum states $\psi$ of perturbations $\hat{\mathcal{T}}, \hat{\mathcal{Q}}$. This strategy is internally consistent and yields a well-defined theory. However, from a conceptual standpoint, it is highly desirable to use a "polymer representation' suggested by the LQG techniques. In cases when the energy density in the perturbations is negligible compared to that in the background, we expect predictions of the polymer representation to reduce to those obtained here using the Fock representation. But if perturbations are described using the polymer representation, quantization of the entire truncated theory would be more firmly rooted in LQG, adding considerable conceptual coherence. In addition, such an extension would also be valuable from mathematical physics considerations. The correspondence between QFT on quantum geometries $\Psi_{o}$ and those on the dressed effective metrics $\tilde{g}_{a b}$ will continue to hold. However, LQG techniques are likely to suggest alternate regularization and renormalization schemes that are tailored to the 'polymer representation'. The adiabatic scheme used here has been carefully developed 
within QFT on cosmological space-times over more than four decades [68, 69, 82-84]. And it handles the ultraviolet divergences satisfactorily. However, as we saw in section II C 2, it does not remove infrared divergences in the case of massless fields. Our current treatment of infrared issues is meant to be a physically motivated but tentative strategy. There should a better procedure that simultaneously handles the ultraviolet and the infrared regime. It is quite possible that such a scheme naturally descends from techniques that are well-adapted to LQG [50]. So far the literature on the relation between LQG and regularization and renormalization in QFT in curved space-times has remained rather general and investigations that focus just on cosmological space-times may well provide richer and more detailed results.

* Initial conditions: In our choice of initial conditions for the background state $\Psi_{o}$ in section III, we were motivated by physical considerations. The new ingredient is the role played by the repulsive force of origin in quantum geometry that causes the quantum bounce. It is significant on a scale of about $10 \ell_{\mathrm{Pl}}$ and effectively dilutes away the inhomogeneities. Since regions of this size at the bounce expand out to become the observable universe at the surface of last scattering, there is a new mechanism to achieve the extraordinary homogeneity that is needed in the initial state to explain the current large scale observations. However, so far the argument is only qualitative. It would be extremely helpful if this could be developed further via concrete calculations, or, evidence for or against this possibility can be gleaned from suitable numerical simulations. Another open issue is that of further narrowing down the initial conditions, especially for $\psi$. The three conditions we imposed are well motivated but still allow a very large class of $\psi$. We used the four choices discussed in section VC to verify that the power spectra are robust and then carried out the detailed numerical simulations using $|\psi\rangle=\left|0^{\text {obv }}\right\rangle$ because there are several reasons in favor of this choice. Are there perhaps additional criteria that will single out this -or another state- essentially uniquely? It would be very helpful even to significantly narrow down the choices by using general physical principles.

Finally, we would like to re-emphasize that in this series of papers we have focused only on the quantum gravity issues. Problems related to particle physics phenomenology and foundational issues related to the possible quantum to classical transition remain.

\section{Acknowledgments}

We have benefited from discussions with and comments and questions from with a large number of colleagues, especially Francois Buchet, David Brizuela, Stefan Hollands, Eiichiro Komatsu, Alok Ladha, Misao Sasaki, Sarah Shandera and David Sloan. This work was supported by the NSF grant PHY-1205388 and the Eberly research funds of Penn state, and the Marie Curie Fellowship program of the EU.

\section{Appendix A: Inclusion of an inflationary potential in the underlying framework}

Since Ref. [19] was addressed to the quantum gravity audience, it focused on mathematical and conceptual issues rather than phenomenological. Therefore, for simplicity of presentation we assumed that the scalar field is massless. In this appendix we summarize the modifications that are necessary to incorporate an inflaton potential, such as 
$V(\phi)=(1 / 2) m^{2} \phi^{2}$ used in the main body of this paper.

The discussion of the homogeneous phase space $\Gamma_{o}$ is the same as that in [19] except that the scalar (or the Hamiltonian) constraint $\mathbb{S}_{o}$ now contains the potential $V(\phi)$ (see Eq (2.2)). The conceptual steps that led to the reduced phase space $\tilde{\Gamma}$ of first order perturbations are also the same as in [19] and the treatment of tensor perturbations in [19] does not require any modifications. However, for scalar perturbations, expressions of the Hamiltonians of the final gauge invariant Mukhanov-Sasaki variables $\mathcal{Q}_{\vec{k}}$ and curvature perturbations $\mathcal{R}_{\vec{k}}$ are now more complicated. Our discussion of the required changes will largely follow Langlois' Hamiltonian treatment [85]. However, because of our primary motivation, we aim at finding expressions of the Hamiltonian that are well suited to loop quantization. Note also that the procedure uses only the constraint equations; we do not assume that the background space-time satisfies full Einstein's equations.

As discussed in [19], the first order constraints generate the gauge transformations for the linear perturbations. One can solve these constraints and isolate gauge invariant variables which suffice to label the points of the reduced phase space $\tilde{\Gamma}_{1}$. As discussed in [19], the Mukhanov-Sasaki variable $\mathcal{Q}_{\vec{k}}$ provides a convenient choice. To arrive at them, note first that, in the notation of [19], the first order metric perturbations $\tilde{\mathfrak{q}}_{a b}(\vec{k})$ can be expanded into scalar, vector modes and tensor modes

$$
\begin{aligned}
\tilde{\mathfrak{q}}_{a b}(\vec{k})= & S_{\vec{k}}^{(1)} \stackrel{\circ}{q}_{a b}+S_{\vec{k}}^{(2)}\left(\hat{k}_{a} \hat{k}_{b}-\frac{1}{3} \stackrel{\circ}{a b}_{a b}\right)+\sqrt{2} V_{\vec{k}}^{(1)} \hat{k}_{(a} \hat{x}_{b)} \\
& +\sqrt{2} V_{\vec{k}}^{(2)} \hat{k}_{(a} \hat{y}_{b)}+\frac{1}{\sqrt{2}} T_{\vec{k}}^{(1)}\left(\hat{x}_{a} \hat{x}_{b}-\hat{y}_{a} \hat{y}_{b}\right)+\sqrt{2} T_{\vec{k}}^{(2)}\left(\hat{x}_{(a} \hat{y}_{b)}\right)
\end{aligned}
$$

where $\hat{k}$ is a unit vector in the $\vec{k}$ direction and $\hat{k}, \hat{x}, \hat{y}$ constitutes a field of orthonormal triads with respect to the comoving fiducial 3 -metric $\stackrel{\circ}{q}_{a b}$ in the momentum space. The Mukhanov-Sasaki variable $\mathcal{Q}_{\vec{k}}$ is then given by

$$
\mathcal{Q}_{\vec{k}}=\varphi_{\vec{k}}-\frac{p_{(\phi)} \gamma}{2 a^{5} \ell^{3} \mathrm{~b}}\left(S_{\vec{k}}^{(1)}-\frac{1}{3} S_{\vec{k}}^{(2)}\right) .
$$

where $\varphi_{\vec{k}}$ is the first order perturbation in the inflaton.

As noted in the main text, dynamics of gauge invariant variables is governed by the part $\mathbb{S}_{2}^{\prime(\mathcal{Q})}$ of the second order constraint obtained by (ignoring the terms that are linear in the second order perturbation but) retaining terms that are quadratic in the first order fields. Since this Hamiltonian is gauge invariant, it can be expressed in terms of the MukhanovSasaki variable $\mathcal{Q}_{\vec{k}}$ and its conjugate momentum $\mathfrak{p}_{\vec{k}}^{(\mathcal{Q})}$ :

$$
\mathbb{S}_{2}^{\prime(\mathcal{Q})}\left[N_{\mathrm{hom}}=a\right]=\frac{1}{2} \int \frac{d^{3} k}{(2 \pi)^{3}}\left[\frac{1}{a^{2}}\left|\mathfrak{p}_{\vec{k}}^{(\mathcal{Q})}\right|^{2}+2\left(\Omega^{2}\right)\left|\mathcal{Q}_{\vec{k}}\right|^{2}\right],
$$

with

$$
\Omega^{2}=-9 \frac{p_{(\phi)}^{4}}{a^{4} \ell^{6} \pi_{(a)}^{2}}+12 \pi G \frac{p_{(\phi)}^{2}}{a^{2} \ell^{6}}-\frac{6 p_{(\phi)} a^{3}}{\pi_{(a)}} \frac{\mathrm{d} V}{\mathrm{~d} \phi}+\frac{a^{4}}{2} \frac{\mathrm{d}^{2} V}{\mathrm{~d} \phi^{2}}+\frac{a^{2} k^{2}}{2} .
$$

Here, we have used the lapse function tailored to the conformal time and the scale factor $a$ and its conjugate momentum $\pi_{(a)}$ normally used in the cosmology literature (translation to the $(\nu, \mathrm{b})$ variables used in $\mathrm{LQC}$ can be readily carried out using Eq. (2.1)). Modulo 
standard factor ordering ambiguities $\Omega^{2}$ can be promoted to a well-defined operator on the background Hilbert space $\mathcal{H}_{o}$.

Another, particularly useful form of $\Omega$ arises from using $\mathbb{S}_{0}=0$ to eliminate the explicit dependence on $\pi_{(a)}$,

$$
\Omega^{2}=\frac{1}{a^{2}}\left(24 \pi G\left(\frac{E_{k} E_{\phi}}{E_{k}+E_{\phi}}\right)-\sqrt{48 \pi G} \sqrt{\frac{E_{k}}{E_{k}+E_{\phi}}} \frac{\mathrm{d} E_{\phi}}{\mathrm{d} \phi}+\frac{1}{2} \frac{\mathrm{d}^{2} E_{\phi}}{\mathrm{d} \phi^{2}}+\frac{a^{4} k^{2}}{2}\right),
$$

where we have defined,

$$
E_{k} \equiv \frac{\mathfrak{p}_{(\phi)}^{2}}{2}, \quad \text { and } \quad E_{\phi} \equiv a^{6} \ell^{6} V(\phi),
$$

which are closely related to the kinetic and potential energy density. Further defining the ratio,

$$
\mathfrak{f} \equiv \frac{6 \kappa E_{k}}{E_{k}+E_{\phi}}=\frac{3 \kappa \mathfrak{p}_{(\phi)}^{2}}{\frac{1}{2} p_{(\phi)}^{2}+a^{6} \ell^{6} V(\phi)},
$$

one finds,

$$
\Omega^{2}=\frac{a^{4}}{2}\left(\mathfrak{f} V(\phi)-2 \sqrt{\mathfrak{f}} V_{, \phi}+V_{, \phi \phi}+\left(\frac{k}{a}\right)^{2}\right) \equiv \frac{a^{2}}{2}\left(\mathfrak{A}+k^{2}\right) .
$$

It is this form of the scalar Hamiltonian that we use in Eq. (2.5).

Finally, as explained in section VA, comparison with observations is simplest in terms of co-moving curvature perturbations $\mathcal{R}_{\vec{k}}$. To arrive at these gauge invariant variables, one can perform a (background dependent) canonical transformation within $\tilde{\Gamma}^{(1)}$, generated by

$$
S=\frac{z}{a} \mathfrak{p}_{\vec{k}}^{(\mathcal{Q})} \mathcal{R}_{\vec{k}}+\frac{1}{2} f \mathcal{R}_{\vec{k}}^{2},
$$

where $f$ and $z$ are arbitrary function of the background phase-space variables to begin with, but determined below. From Eq. (A9) one finds,

$$
\mathcal{R}_{\vec{k}}=\frac{a}{z} \mathcal{Q}_{\vec{k}}, \text { and } \mathfrak{p}_{\vec{k}}^{(\mathcal{R})}=\frac{z}{a} \mathfrak{p}_{\vec{k}}^{(\mathcal{Q})}+f \mathcal{R}_{\vec{k}} .
$$

The choice of $f$ and $z$ is geared to simplify the form of the Hamiltonian in terms of $\mathcal{R}_{\vec{k}}$ and $\mathfrak{p}_{\vec{k}}^{(\mathcal{R})}$. Therefore we will now sketch the procedure and conclude with the explicit form of $z$ that is needed in the expressions (A10) of these quantities. The Hamiltonian in terms of $\mathcal{R}_{\vec{k}}$ and $\mathfrak{p}_{\vec{k}}^{(\mathcal{R})}$ is not used in the main text but may be useful for future investigations.

Recalling that Eq. (A9) is a 'time' dependent canonical transformation, with the evolution given by $\mathbb{S}_{0}$, we find,

$$
\begin{aligned}
\mathbb{S}_{2}^{\prime(\mathcal{R})}\left[N_{\mathrm{hom}}=a\right]= & \frac{1}{2} \int \frac{d^{3} k}{(2 \pi)^{3}}\left(\frac{1}{z^{2}}\left|\mathfrak{p}_{\vec{k}}^{(\mathcal{R})}\right|^{2}+\left(\frac{-2 f}{z^{2}}+\frac{2 a}{z}\left\{\frac{z}{a}, \mathbb{S}_{0}\right\}\right) 2 \operatorname{Re}\left(\mathfrak{p}_{\vec{k}}^{(\mathcal{R})} \mathcal{R}_{\vec{k}}^{\star}\right)\right. \\
& \left.+\left(2 \Omega^{2}\left(\frac{z}{a}\right)^{2}+\frac{f^{2}}{z^{2}}-\frac{2 a f}{z}\left\{\frac{z}{a}, \mathbb{S}_{0}\right\}+\left\{f, \mathbb{S}_{0}\right\}\right)\left|\mathcal{R}_{\vec{k}}\right|^{2}\right)
\end{aligned}
$$


One can check that the equations of motion for $\mathcal{R}$ are independent of the choice of the background function $f$. However, we can choose it to simplify the expression of $\mathbb{S}_{2}^{\prime(\mathcal{R})}$. Specifically, for

$$
f=a z\left\{\frac{z}{a}, \mathbb{S}_{0}\right\}
$$

$\mathbb{S}_{2}^{\prime(\mathcal{R})}$ is diagonal:

$$
\begin{aligned}
\mathbb{S}_{2}^{(\mathcal{R})}\left[N_{\text {hom }}=a\right]= & \frac{1}{2} \int \frac{d^{3} k}{(2 \pi)^{3}}\left(\frac{1}{z^{2}}\left|\mathfrak{p}_{\vec{k}}^{(\mathcal{R})}\right|^{2}\right. \\
& \left.+\left(2 \Omega^{2}\left(\frac{z}{a}\right)^{2}-a^{2}\left\{\frac{z}{a}, \mathbb{S}_{0}\right\}^{2}+\left\{a z\left\{\frac{z}{a}, \mathbb{S}_{0}\right\}, \mathbb{S}_{0}\right\}\right)\left|\mathcal{R}_{\vec{k}}\right|^{2}\right) .
\end{aligned}
$$

Until this point the form of the function $z$ has not been fixed. A particular simplification occurs for the specific choice

$$
z=\frac{-3}{4 \pi G} \frac{p_{(\phi)}}{\pi_{(a)}} \equiv \frac{\gamma}{a^{2} \ell^{3}} \frac{p_{(\phi)}}{\mathrm{b}} .
$$

(This is the relation used in section V). Using this expression, one can explicitly evaluate the various Poisson brackets, for example

$$
\left\{\frac{z}{a}, \mathbb{S}_{0}\right\}=\frac{3 p_{(\phi)}}{a^{2}}-\frac{9 p_{(\phi)}^{3}}{4 \pi G a^{4} \pi_{(a)}^{2}}-\frac{3 a^{3}}{4 \pi G \pi_{(a)}} \frac{\mathrm{d} V}{\mathrm{~d} \phi}
$$

Using this in Eq. (A13) gives

$$
\mathbb{S}_{2}^{\prime(\mathcal{R})}\left[N_{\mathrm{hom}}=a\right]=\frac{1}{2} \int \frac{\mathrm{d}^{3} k}{(2 \pi)^{3}}\left(\frac{1}{z^{2}}\left|\mathfrak{p}_{\vec{k}}^{(\mathcal{R})}\right|^{2}+z^{2} k^{2}\left|\mathcal{R}_{\vec{k}}\right|^{2}\right) .
$$

This form is particularly useful for cosmological calculations, however it is clear from Eq. (A14) that the canonical transformation between $\mathcal{Q}_{\vec{k}}$ and $\mathcal{R}_{\vec{k}}$ is ill defined at points in the trajectory where $p_{(\phi)}=0$ or $\pi_{(a)}=0$. In particular, $p_{(\phi)}$ can vanish during the evolution between the bounce and the onset of slow-roll inflation. Therefore, as discussed in VA, during this phase of evolution $\mathcal{Q}_{\vec{k}}$ is well defined, whilst $\mathcal{R}_{\vec{k}}$ is not.

Finally, as we noted at the end of section II B 1, the presence of the $m^{2} \phi^{2}$ potential also gives rise to some mathematical subtleties in the discussion of the quantum theory of the homogeneous background. These are summarized in section 4.3 of [7] and will be discussed in detail in [56].

[1] A. R. Liddle and D. H. Lyth, Cosmological Inflation and Large-Scale Structure (Cambridge University press, Cambridge (2000)).

[2] S. Dodelson, Modern Cosmology, Academic Press, Amsterdam (2003)).

[3] V. Mukhanov, Physical Foundations of Cosmology, (Cambridge University Press, Cambridge $(2005))$. 
[4] S. Weinberg, Cosmology, (Oxford University press, Oxford (2008)).

[5] D. S. Gorbunov and V. A. Rubokov, Introduction to the Theory of the Early Universe: Cosmological perturbations and Inflationary Theory (World Scientific, Singapore, 2100).

[6] A. Ashtekar and D. Sloan, Probability of inflation in loop quantum cosmology, Gen. Rel. Grav. 43, 3619-3656 (2011).

[7] A. Ashtekar and P. Singh, Loop quantum cosmology: A status report, Class. Quant. Grav. 28, 213001 (2011).

[8] R. H. Brandenberger, Introduction to early universe cosmology, PoS(ICFI 2010)001, arXiv: 1103:2271;

R. H. Brandenberger and J. Martin, Trans-Planckian issues for inflationary cosmology, arXiv: 1211.6753

[9] A. Borde, A. Guth and A. Vilenkin, Inflationary space-times are not past-complete, Phys. Rev. Lett. 90151301 (2003).

[10] X. Chen, M. X. Huan, S. Kachru, and G. Shiu,Observational signature and non-Gaussianities of general single field inflation, JCAP 0701, 002 (2007).

[11] R. Holman and A. Tolley, Enhanced Non-Gaussianity from excited states, JCAP 0805, 001 (2008).

[12] I. Agullo and L. Parker, Non-gaussianities and the Stimulated creation of quanta in the inflationary universe, Phys. Rev. D 83063526 (2011);

Stimulated creation of quanta during inflation and the observable universe Gen. Relativ. Gravit. 43, 2541-2545 (2011).

[13] J. Ganc, Calculating the local-type fNL for slow-roll inflation with a non-vacuum initial state., Phys. Rev. D 84063514 (2011).

[14] I. Agullo, J. Navarro-Salas and L. Parker, Enhanced local-type inflationary trispectrum from a non-vacuum initial state, JCAP 1205, 019 (2012).

[15] J. Ganc and E. Komatsu, Scale dependent bias of galaxies and $\mu$-type distprtion of the cosmic microwave background spectrum from a single field inflation with a modified initial state, Phys. Rev. D 86023518 (2012).

[16] I. Agullo and S. Shandera, Large non-Gaussian halo bias from single field inflation JCAP 09, 007 (2012).

[17] F. Schmidt and L. Hui, CMB power asymmetry from Gaussian modulation, arXiv:1210.2965.

[18] I. Agullo, A. Ashtekar and W. Nelson, A quantum gravity extension of the inflationary scenario, Phys. Rev. Lett. 109251301 (2012).

[19] I. Agullo, A. Ashtekar and W. Nelson, An extension of the quantum theory of cosmological perturbations to the Planck era, Phys. Rev. D 87043507 (2013).

[20] M. Bojowald, Absence of singularity in loop quantum cosmology, Phys. Rev. Lett. 86 52275230 (2001).

[21] A. Ashtekar, M. Bojowald and J. Lewandowski, Mathematical structure of loop quantum cosmology, Adv. Theo. Math. Phys. 7 233-268 (2003)

[22] A. Ashtekar, T. Pawlowski and P. Singh, Quantum nature of the big bang, Phys. Rev. Lett. 96 141301 (2006),

[23] A. Ashtekar, T. Pawlowski and P. Singh, Quantum nature of the big bang: An analytical and numerical investigation I, Phys. Rev. D 73124038 (2006).

[24] A. Ashtekar, T. Pawlowski and P. Singh, Quantum nature of the big bang: Improved dynamics, Phys. Rev. D 74084003 (2006).

[25] A. Ashtekar, A. Corichi and P. Singh, Robustness of predictions of loop quantum cosmology, 
Phys. Rev. D 77024046 (2008).

[26] P. Singh, Are loop quantum cosmologies never singular? Class. Quant. Grav. 26125005 (2009).

[27] A. Ashtekar, T. Pawlowski, P. Singh and K. Vandersloot, Loop quantum cosmology of k=1 FRW models, Phys. Rev. D 75024035 (2006).

[28] L. Szulc, W. Kaminski, J. Lewandowski, Closed FRW model in loop quantum cosmology, Class. Quant. Grav. 242621 (2007).

[29] E. Bentivegna and T. Pawlowski, Anti-deSitter universe dynamics in LQC, Phys. Rev. D 77 124025 (2008).

[30] W. Kaminski and T. Pawlowski, The LQC evolution operator of FRW universe with positive cosmological constant, Phys. Rev. D 81024014 (2010).

[31] T. Pawlowski and A. Ashtekar, Loop quantum cosmology with a positive cosmological constant, Phys. Rev. D 85, 064001 (2012).

[32] A. Ashtekar and E. Wilson-Ewing, Loop quantum cosmology of Bianchi type I models, Phys. Rev. D 79083535 (2009).

[33] M. Martin-Benito, G. A. Mena Marugan, T. Pawlowski, Loop quantization of vacuum Bianchi I cosmology, Phys. Rev. D 78064008 (2008);

Physical evolution in loop quantum cosmology: The example of vacuum Bianchi I, Phys. Rev. D 80084038 (2009).

[34] A. Ashtekar and E. Wilson-Ewing, Loop quantum cosmology of Bianchi type II models, Phys. Rev. D 80123532 (2009).

[35] E. Wilson-Ewing, Loop quantum cosmology of Bianchi type IX models, Phys. Rev. D 82 043508 (2010).

[36] M. Martin-Benito, L. J. Garay and G. A. Mena Marugan, Hybrid quantum Gowdy cosmology: combining loop and Fock quantizations, Phys. Rev. D 78083516 (2008);

L. J. Garay, M. Martn-Benito, G. A. Mena Marugan, Inhomogeneous loop quantum cosmology: Hybrid quantization of the Gowdy model, Phys. Rev. D 82044048 (2010).

[37] D. Brizuela, G. A. Mena Marugan and T. Pawlowski, Big bounce and inhomogeneities, Class. Quant. Grav. 27052001 (2010).

[38] M. Martin-Benito, G. A. Mena Marugan, E. Wilson-Ewing, Hybrid quantization: From Bianchi I to the Gowdy model, Phys. Rev. D 82084012 (2010).

[39] M. Martn-Benito, D. Martin-de Blas, G. A. Mena Marugan, Matter in inhomogeneous loop quantum cosmology: the Gowdy $T^{3}$ model, Phys. Rev. D 83084050 (2011).

[40] D. Brizuela, G. A. Mena Marugan and T. Pawlowski, Effective dynamics of the hybrid quantization of the Gowdy T3 universe, Phys. Rev. D 84124017 (2011).

[41] M. Bojowald, Loop quantum cosmology, Liv. Rev. Rel. 811 (2005).

[42] A. Ashtekar, J. Baez, A. Corichi, and K. Krasnov, Quantum Geometry and Black Hole Entropy, Phys. Rev. Lett. 80, 904-907 (1998).

[43] A. Ashtekar, J. Baez and K. Krasnov, Quantum Geometry of Isolated Horizons and Black Hole Entropy, Advances in Theoretical and Mathematical Physics 4, 1-95 (2000).

[44] J. F. Barbero G., J. Lewandowski and E. J. S. Villasenor, Quantum isolated horizons and black hole entropy, arXiv:1203.0174.

[45] E. Bianchi, E. Magliaro and C. Perini, LQG propagator from new spin foams, Nucl. Phys. B 822, 245-269 (2009);

E. Bianchi and Y. Ding, Lorentzian spin-foam propagator, Phys. Rev. D 86, 104040 (2012).

[46] C. Rovelli, Zakopane lectures on loop gravity, arXiv:1102.3660. 
[47] M. Fernandez-Mendez, G. A. Mena Marugan, and J. Olmedo, Hybrid quantization of an inflationary universe, Phys. Rev. D 86, 024003 (2012).

[48] A. Ashtekar, W. Kaminski and J. Lewandowski, Quantum field theory on a cosmological, quantum space-time, Phys. Rev. D 79064030 (2009).

[49] A. Ashtekar and J. Lewandowski, Background independent quantum gravity: A status report, Class. Quant. Grav. 21 R53-R152 (2004).

[50] T. Thiemann, Introduction to Modern Canonical Quantum General Relativity. (Cambridge University Press, Cambridge, (2007)).

[51] C. Rovelli and L. Smolin, Discreteness of area and volume in quantum gravity, Nucl. Phys. B 442 593-622 (1995); Erratum: Nucl. Phys. B 456753 (1995).

[52] A. Ashtekar and J. Lewandowski, Quantum theory of geometry I: Area operators, Class. Quant. Grav. 14 A55-A81 (1998)

A. Ashtekar and J. Lewandowski, Quantum theory of geometry II: Volume Operators, Adv. Theo. Math. Phys. 1 388-429 (1997).

[53] T. Thiemann, A length operator for canonical quantum gravity, J. Math. Phys. $393372-3392$ (1998).

[54] E. Bianchi, The length operator in loop quantum gravity, Nucl. Phys. B 807 591-624 (2009).

[55] Y. Ma, C. Soo and J. Yang, New length operator for loop quantum gravity, Phys. Rev. D 81 124026 (2010)

[56] A. Ashtekar, T. Pawlowski, P. Singh, (in preparation)

[57] L. Parker and S. A. Fulling, Adiabatic regularization of the energy-momentum tensor of a quantized field in homogeneous spaces, Phys. Rev. D 9 341-354 (1974).

[58] D. Marolf, Refined algebraic quantization: Systems with a single constraint. arXive: gr-qc/9508015; Quantum observables and recollapsing dynamics, Class. Quant. Grav. 12 1199-1220 (1994).

[59] A. Ashtekar, J. Lewandowski, D. Marolf, J. Mourão and T. Thiemann, Quantization of diffeomorphism invariant theories of connections with local degrees of freedom, J. Math. Phys. 36 6456-6493 (1995).

[60] A. Ashtekar, L. Bombelli and A. Corichi, Semiclassical states for constrained systems, Phys. Rev. D72 025008 (2005).

[61] S. W. Hawking and G. F. R. Ellis, Large scale structure of space-time (Cambridge University Press, Cambridge, 1973).

[62] V. Taveras, LQC corrections to the Friedmann equations for a universe with a free scalar field, Phys. Rev. D 78064072 (2008).

[63] A. Ashtekar, M. Campiglia and A. Henderson, Path Integrals and the WKB approximation in loop quantum cosmology, Phys. Rev. D 82124043 (2010).

[64] E. Calzetta, M. Sakellariadou, Inflation in inhomogeneous cosmology, Phys. Rev. D 452802 (1992).

[65] E. Calzetta, M. Sakellariadou, Semiclassical effects and the onset of inflation, Phys. Rev. D 47 3184 (1994).

[66] C. Rovelli, Quantum mechanics without time: A model, Phys. Rev. D 422638 (1990).

[67] M. Bojowald, Philipp A. Hoehn, and A. Tsobanjan, An effective approach to the problem of time Class. Quant. Grav. 28035006 (2011).

[68] L. Parker, The creation of particles in an expanding universe, Ph.D. thesis, Harvard University (1966).

[69] L. Parker and S. A. Fulling, Adiabatic regularization of the energy momentum tensor of a 
quantized field in homogeneous spaces, Phys. Rev. D 9341 (1974).

[70] L. Parker, Particle creation in expanding universes, Phys. Rev. Lett. 21562 (1968); Quantized fields and particle creation in expanding universes I, Phys. Rev. 183, 1057(1969).

[71] P. Bielewicz and A. J. Banday, Constraints on the topology of the universe derived from the 7-year WMAP data, MNRS 412, 2104-2110 (2011).

[72] E. Komatsu et al, Seven-year Wilkinson micowave anisotropy probe (WMAP) observations: Cosmological interpreation, Astrophys. J. Suppl. Ser. 192, 18 (2011).

[73] R. Penrose, Singularities and time-asymmetry, in General Relativity: An Einstein Centenary Survey, edited by S. W. Hawking and W. Israel. (Cambridge University Press, Cambridge, 1979), pages 581 to 638 .

[74] A. Ashtekar, I. Agullo and W. Nelson (in preparation).

[75] J. Martin, A. Riazuelo, M. Sakellariadou, Nonvacuum initial states for cosmological perturbations of quantum-mechanical origin, Phys. Rev. D 61, 083518 (2000).

[76] I. Agullo and W. Nelson, Preferred quantum states in linear QFT on FLRW Backgrounds. (In preparation).

[77] R. Easther, B. R. Greene, W. H. Kinney and G. Shiu, A Generic estimate of transPlanckian modifications to the primordial power spectrum in inflation, Phys. Rev. D 66, 023518 (2002); Imprints of short distance physics on inflationary cosmology, Phys. Rev. D 67, 063508 (2003); Inflation as a probe of short distance physics, Phys. Rev. D 64, 103502 (2001).

[78] U. H. Danielsson, A Note on inflation and transPlanckian physics, Phys. Rev. D 66, 023511 (2002).

[79] V. F. Mukhanov, L. Raul W. Abramo and Robert H. Brandenberger, Backreaction Problem for Cosmological Perturbations, Phys. Rev. Lett. 78, 1624 (1997)

[80] L. Linsefors, T. Cailleteau, A. Barrau and J. Grain, Primordial tensor power spectrum in holonomy corrected Omega-LQC, arXiv:1212.2852.

[81] E. Ranken and P. Singh, Non-singular power-law and assisted inflation in loop quantum cosmology, Phys. Rev. D 85, 104002 (2012).

[82] P. R. Anderson, C. Molina-Paris, and E. Mottola, Short distance and initial state effects in inflation: Stress tensor and decoherence, Phys. Rev. D 72043515 (2005).

[83] S. Fulling, Aspects of Quantum Field Theory in Curved Space-times, (Cambridge UP, Cambridge, 1989).

[84] L. Parker and D. Toms, Quantum Field Theory in Curved Space-time (Cambridge UP, Cambridge, 2009).

[85] D. Langlois, Hamiltonian formalism and gauge invariance for linear perturbations in inflation, Class. Quant. Grav. 11 389-407 (1994). 